\title{
Oklahoma
}

\section{Native Plant Record}

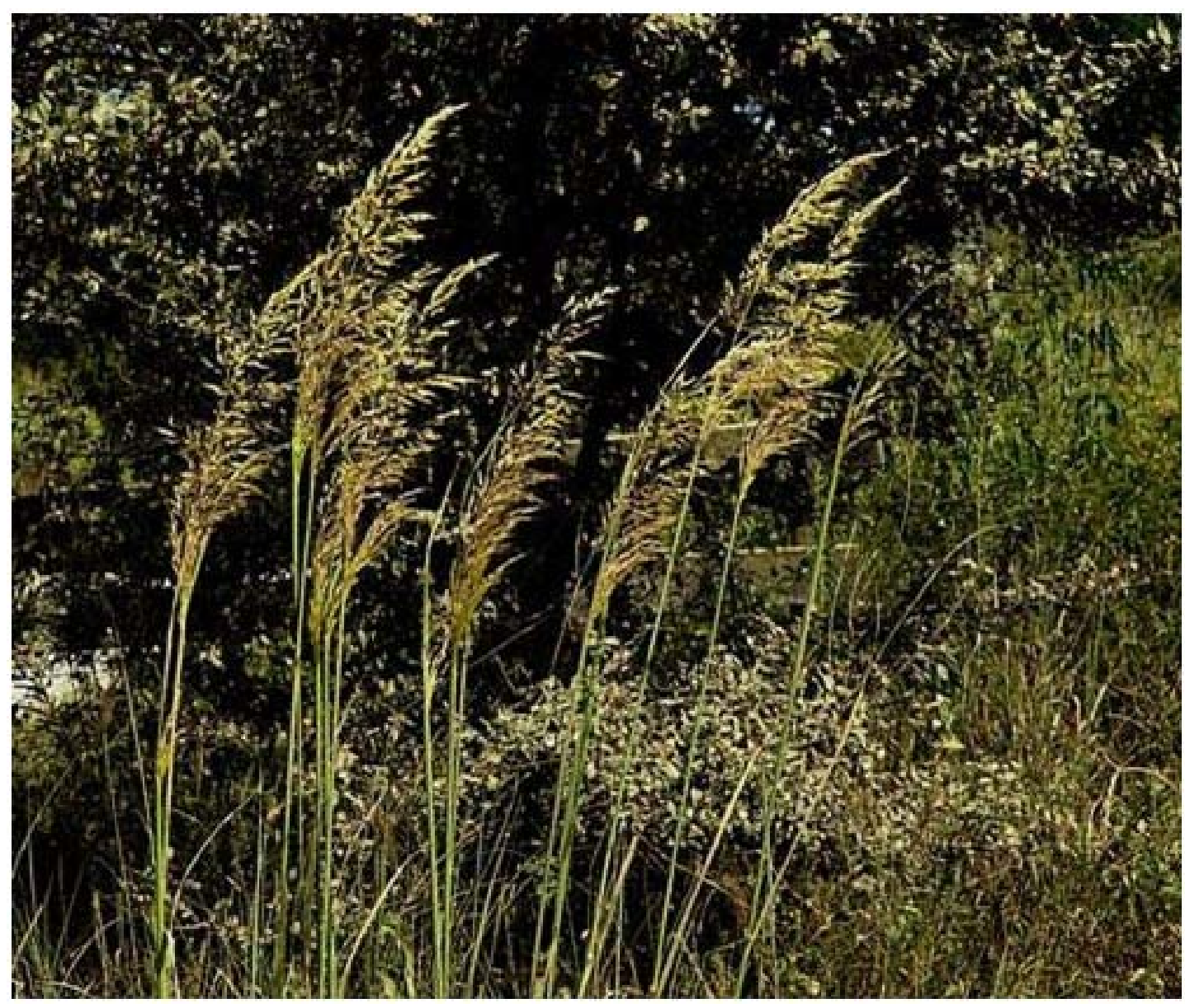

Journal of the Oklahoma Native Plant Society Volume 4, Number 1, December 2004 


\title{
Oklahoma Native Plant Record \\ Journal of the Oklahoma Native Plant Society \\ 2435 South Peoria \\ Tulsa, Oklahoma 74114
}

Volume 4 Number 1, December 2004

ISSN 1536-7738

\author{
Managing Editor, Sheila A. Strawn \\ Technical Editor, Patricia Folley \\ Technical Advisor, Bruce Hoagland \\ CD-ROM Producer, Chadwick Cox \\ Website: http://www.usao.edu/ onps/
}

The purpose of the ONPS is to encourage the study, protection, propagation, appreciation and use of the native plants of Oklahoma. Membership in ONPS shall be open to any person who supports the aims of the Society. ONPS offers individual, student, family, and life membership.

2004 Officers and Board Members

President: James Elder

Vice-president: Constance Murray

Secretaries:

Kimberly A. Shannon

Tina Julich

Treasurer: Mary Korthase

Past President: Patricia Folley

Board Members:

Paul Buck

Kay Gafford

Melynda Hickman

Monica Macklin

Elfriede Miller

Stanley Rice

Northeast Chapter Chair: Constance Murray

Central Chapter Chair: Sharon McCain

Cross-timbers Chapter Chair:

Suzanne McAllister

Mycology Chapter Chair: Clark Ovrebo

Historians: Carla and Dale Chlouber

Ann Long Award Chair: Patricia Folley

Harriet Barclay Award Chair:

Constance Taylor
ONPS Service Award Chair: Sue Amstutz

Conservation Chair: Chadwick Cox

Publicity Chair:

Publications Co-chairs:

Sheila Strawn

Constance Taylor

Marketing Co-chairs:

Lawrence Magrath

Susan Chambers

Photo Contest Co-chairs:

Patricia and Chadwick Cox

Newsletter Editor: Chadwick Cox

Librarian: Bonnie Winchester

Website Manager: Chadwick Cox

Mailing Committee Chair: Karen Haworth

Color Oklahoma Committee Chair:

Constance Murray

Wildflower Workshop Chair: Larry Magrath

Cover photo: Sorghastrum nutans, Indian

grass, our state grass by Charles Lewallen

Articles (c) The Authors

Journal compilation (c) Oklahoma Native Plant Society

Except where otherwise noted, this work is licensed under a Creative Commons Attribution-NonCommercialShareAlike4.0 International License, https://creativecommons.org/licenses/by-nc-sa/4.0/, which permits unrestricted use, distribution, and reproduction in any medium, provided the original work is properly attributed, not used for commercial purposes, and, if transformed, the resulting work is redistributed under the same or similar license to this one.

https://doi.org/10.22488/okstate.17.100025 


\section{Oklahoma Native Plant Record Volume 4, Number 1}

\section{Table of Contents}

Forward

Mr. James F. Elder, ONPS President

Ecological Factors Affecting the Distribution of Woody Vegetation

Near the Arkansas River, Tulsa County with Special Reference

to the Smoke-tree.

Masters Thesis of Dr. Anne Wanamaker Long

Cotinus obovatus Raf. (Smoke-tree) in Oklahoma

Dr. Bruce W. Hoagland

Giant Cane and Southeastern Indian Baskets

Wichita Mountains Wildlife Refuge Survey 2000

Ms. Julia A. Jordan

Vascular Flora of the Chouteau Wildlife Management Area

Wagoner County, Oklahoma..

Dr. Bruce W. Hoagland and Dr. Forrest L. Johnson (dec.)

Status and Habitat Characteristics of Cypripedium kentuckiense

(Kentucky lady's slipper) in Southeastern Oklahoma

Ms. Amy K. Buthod and Dr. Bruce W. Hoagland

Common Lawn and Garden Fungi of Central Oklahoma

Dr. Clark L. Ovrebo

Critic's Choice Essay: Support Your Local Herbarium.

Dr. Wayne J. Elisens

Editorial: Why Do Species' Names Change?

Ms. Patricia A. Folley 


\section{Foreword}

It was a pleasure to learn that Anne Long's thesis on the distribution and ecology of the American smoketree, Cotinus obovatus was to be published in the this issue of the Journal of the Oklahoma Native Plant Society.

I was first introduced to Anne and the American smoke-tree in the spring of 1970 while a student in Dr. Harriet Barclay's ecology class. Anne was one of Dr. Barclay's last graduate students at the University of Tulsa. She was a frequent guest on many of our class fieldtrips, especially to the Redbud Valley Preserve in Eastern Oklahoma. I fondly remember Anne and Dr. Barclay introducing us to C. obovatus, describing its characteristics and distribution to the class. Anne's research indicated that this species can often be found distributed atop many of Oklahoma's limestone bluffs, up and down the Arkansas, and other eastern Oklahoma rivers. To this day, I often look for the smoke-tree whenever I am near such a bluff on my many ONPS fieldtrips.

This tree is described by the U.S. Forest Service as a hardy species with beautiful fall foliage and few pests or diseases. They recommend it as a potential attractive native ornamental, but do admit that it is somewhat difficult to get established.

Anne Long's contributions to Oklahoma's' flora was tragically cut short by cancer and one can only guess as to what her future contributions may have been. We currently live in some exciting times, with DNA analysis providing new insights into our knowledge of species relationships. This knowledge still needs to be supported by fieldwork in species distribution and ecology. Hopefully, there will be future graduate students and other researchers that will continue to carry on Anne's legacy and add to our knowledge of Oklahoma's unique geography, flora, and fauna.

James Elder ONPS President

June 2004 


\title{
Ecological Factors Affecting the Distribution of Woody Vegetation Near the Arkansas River, Tulsa County with Special Reference to the Smoke-tree Cotinus obovatus
}

\author{
Anne Wanamnaker Long \\ A thesis submitted in partial fulfillment of the requirements \\ for the degree of Master of Science in the Department of Botany \\ The University of Tulsa 1970
}

ABSTRACT

Ecological factors affecting plant distribution were studied over different rock strata and slope exposures above the Arkansas River, Tulsa County. Here the Wann sandstone caprock is underlain by the lola limestone formation. The vegetation was analyzed taxonomically by a complete collection throughout one growing season. Belt transects crossing rock strata on all slope exposures permitted computation of parameters summarized by an Importance Percentage for each woody species. Differences in species populations and degree of mesophytism exist on the slope exposures. Sandstone upland dominants are post and blackjack oaks. Smoke-tree, rare in Oklahoma, and chinquapin oak are closely associated in limestone microhabitats, where each occupies a separate niche. The smoke-tree, of disjunct distribution, appears to be a relict of widespread occurrence in past geologic periods. Its survival with limited ecological amplitude is due to the continuance of the microhabitats to which it is so well adapted.

\section{INTRODUCTION}

As is evident to even the most casual observer, there is often a marked correlation between the vegetative cover of the land and the rock strata which underlie it. Abrupt changes in vegetation may well indicate distinct changes in subsurface geological formation. This fact has given rise to the rather recent science of Indicator Geobotany. Russian scientists, particularly, are utilizing vegetational analyses as a rapid, inexpensive and reasonably accurate means of evaluating soil, terrain, and hydrogeological conditions. Aerial surveys have made possible the widespread use of these methods over vast expanses of largely inaccessible land (Chikishev 1961).

Piqued by frequent field trips and residences in various sections for the country, the author has become intrigued by this trend to meld the disciplines of botany and geology into an inter-related science with certain universal applications. The purpose of this investigation was to study an area encompassing differing rock strata in order to note possible correlations between the vegetation and the underlying rock formations. A thorough examination of the Geologic Map of Tulsa County, as compiled by Malcolm Oakes in 1952, led to several possible sites. The one selected is in the southwest one fourth of Section 1, T.19 N, R.10 E, 15 miles west of Tulsa (Figure 1). It is about four miles below the confluence of the Cimarron and Arkansas Rivers whose waters are today impounded by Keystone Dam, completed in 1968 by the United States Corps of Engineers.

The location is of interest botanically, since Thomas Nuttall, the early nineteenth century botanist, embarked on the Cimarron River west of this point and proceeded down the Arkansas a short distance before returning to Fort Smith during his travels through Arkansas Territory. In his journal of September 9, 1919, he wrote, "About noon we arrived at the entrance of the Arkansa (sic) and were gratified with the taste of fresh water. Here the stream, now at its lowest depression was almost colorless and scarcely anywhere exceeding the depth of 3 feet. We traveled down it 9 or 10 miles and saw the ascending smoke of the Osages whom if possible we wished to avoid" (Thwaites 1904-07). It may also be noted that Washington Irving and his party camped at a point on the Arkansas River, known as Bear's Glen, one and a half miles above the mouth of the Cimarron River in 1832 (Barclay 1947).

\section{METHODS}

Knowledge of the geology and identification of the rocks exposed in the area were obtained

Long, A.W.

https://doi.org/10.22488/okstate.17.100027 
from Oakes' (1952) detailed study of the character, distribution, and thickness of the formations that outcrop in Tulsa County. Mr. Allan P. Bennison, consulting geologist of Tulsa, confirmed the rock formations in the field.

Climatological data were obtained from the United States Weather Bureau office at Tulsa International Airport. Precipitation and temperature figures are based on records dating back to 1931 .

A series of soil samples was taken from the upland where the parent rock consists of sandstone, and from the accumulated soil were obtained by means of a soil auger. They were analyzed by the Tulsa County Agricultural agent for organic matter and $\mathrm{pH}$.

Analysis of woody vegetation was made by belt transects which crossed the rock strata on the different slope and bluff exposures. Weaver and Clements (1938) suggest the use of such transects for analysis of vegetational changes due to differences in environment such as are caused by slope exposure or other irregularities in topography or soil. Six transects five meters wide were made, one each on the south, east, and west slopes and three on the north bluff. Because of the varying widths of the strata exposed, the transects ranged in length from 62 to 108 meters. All trees and shrubs in these transects were recorded and the density, frequency, and basal area were determined for those with a diameter breast high (DBH) of three inches or more. These parameters were used to obtain the relative density, relative frequency and relative basal area of each species in comparison with the other species. The sum of the latter three figures, divided by three, determined the importance percentage of a species (Rice and Penfound 1959). The data were used in comparing the tree species on different slopes and in detecting changes as the transects passed over different rock formations.

Collections and determinations of all vascular plants occurring in the area were made over an entire growing season and are presented in Table I. Weekly trips to the study site were made to collect specimens at the time of blooming. Correlation of the vegetation with the underlying strata and with slope exposure has been corroborated by habitat records of the collections. All specimens are deposited in the University of Tulsa Herbarium

\section{CLIMATE}

The climate of Tulsa County is essentially continental with sudden temperature changes and occasional severe storms. Winters are considered to be mild and temperatures are often above 100 degrees $F$. The average yearly rainfall is 37.25 inches, most of which falls during the long growing season. The prevailing winds are southerly at a yearly average of 10.7 miles per hour. Violent wind storms and tornadoes may occur, particularly during spring and early summer.

For a graphic depiction of Weather Bureau data from 1931 to 1968 see Figure 2. It is evident that the overall development of vegetation is closely related to the climatic features shown. The rainfall is greatest in the spring of the year, thus permitting a fairly luxuriant growth of vegetation. By the end of summer, however, many spring-flowering species have completed their growth cycles. The vegetation then appears notably less dense with an abundance of grasses and late-flowering herbs.

\section{GEOLOGY, TOPOGRAPHY AND SOILS}

The rocks exposed in Tulsa County are predominantly shales, interspersed with beds of limestone and sandstone, all of which were deposited during the middle and upper Pennsylvanian Period of the Paleozoic Era. They are a part of the sequence of Pennsylvanian rocks so well exposed in the northern mid-continent region. Having been subsequently uplifted and tilted, they now dip in a direction slightly north of west, at rates ranging form 30-50 feet per mile (Oakes 1952).

The present topography is the result of erosion, mainly by water, which has worn away the soft shales and produced extensive plains with the eroded edges of the more resistant sandstones and limestones forming eastward-facing cuestas or escarpments overlooking the plains. West of Tulsa, where the section contains a greater percentage of hard sandstone, erosion is less advanced. The streams have generally cut narrow valleys which are flanked by steep-sided hills, broken by cliffs and protruding ledges formed by the resistant beds of limestone and sandstone. Drainage of the county is by means of the Arkansas River and its tributaries. In the vicinity of this study, the Arkansas River is deeply incised (Figure 3), the floodplain being less than two miles wide, and the hills on both sides of the river rising precipitously $200-230$ feet. 
The area encompasses a high bluff on the south bank of the river (Figure 4). Here the limestone, classified as the lola formation, is conformably overlain and capped by a massive sandstone, the Wann formation. The Wann sandstone is approximately 30 feet thick, and the lola formation is 60 feet thick (Figure 5). The lola consists of upper and lower limestone members interbedded with shale. The limestone is very irregular and sandy, and the intervening shale layer is obscured by talus. Due to the greater erosion of the softer shale huge masses of the upper limestone member have broken from the bluff and rest as slump blocks on the slopes (Figure 6). In addition, the Wann sandstone caprock fractures into large blocks on the top of the bluff (Figure 7).

The lola formation rests on the Chanute sandstone, which is largely covered by debris from the formation above it and is poorly exposed.

The soil analyses (Table II) indicate that the Wann sandstone is strongly acid. The very shallow soil mantle, the decomposition of the leaves of the dominant oaks, and the leaching of carbonates all tend to contribute to lowering the $\mathrm{pH}$. Although the $\mathrm{pH}$ of the limestone ledge is approximately neutral, there is a slight increase in $\mathrm{pH}$ on the shale below, undoubtedly due to the leaching of the carbonates from the limestone layer.

The analyses also show an increased accumulation of organic matter from the upland to the bottom of the slopes. This can be attributed to erosion from the upland and the more abundant vegetation on the limestone and shale.

For better understanding of the influence of the soil on plant distribution, an investigation of water and mineral relationships should be made. Certain conclusions, however, can be drawn on the basis of the characteristics of the strata. It seems evident that the water moves readily through the sandstone caprock, thus limiting the vegetation on the upland to those species of low moisture requirements. The limestone very likely serves as an aquifer supplying ample moisture to support a dense vegetation, with the underlying shale, because of its small pore size, serving to retard the passage of water.
Since many of the limestone formations in Tulsa County are known to contain phosphatic nodules in significant amounts, it is probable that they are present to some extent in the lola and may be a contributing factor in determining the vegetation on the slopes.

\section{Taxonomic Analysis}

\section{VEGETATION}

One hundred and eighty species of vascular plants were collected at the study site which covers an area of approximately 50 acres. This collection represents a total of 134 genera and 54 families (Table I). The variety of the vegetation and the seasonal changes in plant development were apparent on weekly trips to the area. These visits also revealed the segregation of certain species into distinct communities, coinciding with abrupt changes in topography, and they served to illustrate the dynamics of plant growth and reproduction. Upland

The sandstone upland is characterized by the oak-hickory woodland of the sandstone hills area (Bruner 1931). Here the dominants are post oak (Quercus stellata) and black jack oak (Q. marilandica). Texas hickory (Carya texana) occurs infrequently and is of little importance.

Since there are few other woody species on the upland, there is a definite uniformity of the stand as may be seen in Figure 7. Tree species with greater moisture requirements are unable to survive and reproduce.

Rice and Penfound's study of the upland forests of Oklahoma (1959) concurs with these findings. They listed post oak and black jack oak as the dominants for Tulsa County. Barclay (1947) gives post oak as the single dominant on the Wann sandstone above Bear's Glen, Pawnee County, some four miles from this site.

\section{Slopes and Bluff}

The vegetation on the slopes is in marked contrast to the upland in that many species occur and they provide a more varied cover. The west slope which forms one side of a wide ravine that drains the area is rather gentle. Chinquapin oak (Q. muehlenbergii), and smoke-tree (Cotinus obovatus) are the most significant plants. They appear near the top of the slope on the limestone ledge. Among the other trees are post oak, red haw (Crataegus sp.), persimmon (Diospyros virginiana), sand plum (Prunus angustifolia), white ash (Fraxinus 
americana), and rough leaf dogwood (Cornus Drummondii). An abundant grass cover occurs beneath and between the trees of the west slope.

The ravine on the east is steeper and more protected; the vegetation is, therefore, denser. Chinquapin oak and smoke-tree are prominent along the limestone ledge. On other parts of the slope are post oak, white ash, Texas hickory, shadbush (Amelanchier arborea), and Shumard's oak (Quercus shumardii). Open areas covered with grasses made up a smaller proportion than on the west exposure.

The heaviest vegetation occurs on the steep north bluff (Figure 8). Here shrubs, trees, and vines form an almost impenetrable thicket early in the growing season. Chinquapin oak and smoke-tree dominate the upper limestone ledge. Along the lower limestone outcrop, chinquapin oak is largely replaced by Shumard's oak growing in association with the smoke-tree. The Oaks are large and impressive as shown in Figure 9. The smoke-tree grows in big clumps with many trunks arising from the base (Figure 10). Other trees on the north face are redbud (Cercis canadensis), American elm (Ulmus americana), slippery elm (U. rubra), Texas hickory, bitternut hickory (Carya cordiformis), white ash, and rough leaf dogwood. Grasses occur in an open band between the limestone ledges.

The sandstone caprock on the south is extensive and slopes very gradually with no marked change in the vegetation until the limestone is exposed. Here hackberry (Celtis laevigata), blackhaw (Viburnum prunifolium), and rough leaf dogwood are present.

In general, the vegetation at this study site corresponds rather closely with that at Bear's Glen, as reported by Barclay (1947). A notable difference is the complete absence of Cotinus at Bear's Glen where no limestone was present. (The area is now submerged by Keystone Reservoir.) Red cedar (Juniperus virginiana), which was an important species at Bear's Glen, is of little significance here. Only two were encountered in this area.

\section{RELATIONSHIP OF VEGETATION TO} UNDERLYING STRATA

Analysis of Woody Vegetation

Since it was impossible to run the south transect from the top of the bluff due to the presence of a small oil field at this point, the transect was made to the east, on the south flank of an adjacent bluff (Figure 4). Because of the slight grade of the slope, this transect should probably be considered as part of the upland. The difference in elevation between the beginning of the transect and the limestone outcrop is not sufficient to cause perceptible changes in vegetation. The dominant trees here are post oak and black jack oak. The importance percentage (I.P.), computed from the transect are 65.9 for the post oak and 41.4 for the black jack oak. Rice and Penfound (1959) consider an importance percentage of 25 or more an indication of dominance.

It was evident, however, from the data obtained from the west, east and north transects that, here, both slope exposure and underlying strata are factors in determining the woody vegetation. The transects are arranged in Figure 11 so as to correlate the upper limestone outcrop on the three exposures. As will be noted from the chart, three transects were made on the north-facing bluff because of the greater variety and density of the vegetation on the north. The chart also shows that the lower limestone member outcrops only on the steep north face and is not reached on the east or the west.

The tree species with a DBH of three inches or more are plotted where they occur within the transects and their diameters are recoded. All species under three inches DBH are considered as reproduction and their counts listed (Figure 11). The presence of multi-trunked Cotinus is indicated within the quadrats but since the diameters of the individual trunks were under three inches, their basal areas were not determined. For this reason, although the chart shows that the smoke-tree is confined almost exclusively to the limestone strata, its relative importance and I.P. cannot be computed.

The data in Table III indicate that the three oaks: post, chinquapin, and Shumard's, are dominants on the north-facing bluff but that each occupies a particular habitat on the bluff. Post oak, dominant on the sandstone upland, is largely replaced by chinquapin oak on the upper limestone ledge, and this species is replaced by Shumard's oak on the lower limestone outcrop.

It would seem that this segregation of the oaks is due in part to moisture requirements. The post oak is best adapted to the conditions on the dry upland, chinquapin oak to the more 
mesophytic habitat on the upper part of the slope, and Shumard's oak requiring the very moist conditions below. The complete absence of Shumard's oak on the west and south exposures gives further evidence of its high moisture requirements; whereas chinquapin oak occurs sparingly on the west slope and is absent on the south.

The occurrence of post oak on the limestone on the east and west-facing slopes and on the east end of the bluff is somewhat misleading, for here erosion has dissected the limestone ledge into large blocks, with small ravines between the blocks. It is in the open, well-drained sites that post oak occurs.

Since white ash occurs almost exclusively below the limestone strata, it seems apparent that it cannot survive the aridity of the upland.

Reproduction counts on the transect chart are significant, for they suggest that each community is continuing to maintain itself. The preponderance of small post oaks on the limestone upland leaves little doubt that it will continue to dominate this location.

As the gaps between the limestone blocks on the east and west slopes enlarge and provide greater open areas for evaporation and runoff, the post oak, already established, will increase. Over a long period of time the decline and eventual elimination of the smoke-tree and chinquapin oak on these slopes seem assured. They will then probably be confined to the north-facing bluff.

\section{Herbaceous Vegetation}

It is evident from this investigation that a number of herbaceous plants are restricted to very particular habitats. Two small ferns, Notholaena dealbata and Pellea atropurpurea, although abundant, were found only in crevices on the limestone ledges or on the limestone slump blocks. Other species confined to the limestone outcrops are: Yucca arkansana, Gerardia heterophylla, G. tenuifolia, Penstemon cobea, Cleome serrulata, Rosa setigera, R. carolina, Euphorbia hexagona, Ceanothus americanus, Liatris punctata, and $L$. squarrosa. Herbaceous species limited to the sandstone were markedly fewer in number. They include Commelina erecta, Sedum nuttallianum, Tephrosia virginiana, Lechea tenuifolia, Penstemon tubaeflorus, and Antennaria plantaginifolia. Although no attempt was made to tabulate herbaceous materials on the basis of underlying strata, the collections indicate that the largest number of species was to be found on the talus slopes where the shale is covered with soil and with limestone and sandstone debris from the formations above.

\section{THE IMPORTANCE OF Cotinus obovatus}

\section{Taxonomy}

Cotinus obovatus, or smoke-tree, is a small tree or large shrub up to 35 feet tall, in the family Anacardiaceae. It has alternate, simple, entire, obovate leaves four to six inches long and two to three inches wide, which form dense summer foliage (Figure 12). The plants are usually dioecious with small flowers, many abortive, in loose terminal thyrses. The slender pedicels elongate after flowering and those on the abortive flowers become plumose-villous. The name "smoke-tree" alludes to the "smoky" appearance of the inflorescences. The species tends to grow in clumps and to reproduce asexually by means of root or stump sprouts.

The genus consists of three or more species found in Eurasia and eastern North America. It is represented in the western hemisphere by the single species, $C$. obovatus, of interrupted distribution in southern United States. Although Cotinus was originally classified with Rhus, recent studies indicate that the two genera are not closely related. According to Engler, Cotinus represents a branch of evolution different from but having a common origin with that of Rhus (Brizicky, 1962). The European species, C. Coggygria, because of its more feathery panicles, is cultivated as a popular ornamental in the United States and Europe.

Distribution

The American smoke-tree is found in disjunct populations on wooded, rocky cliffs and river bluffs, generally on lime stone. It occurs in the mountains of Tennessee, in Daviess County, Kentucky, where it may have been introduced, in southwestern Missouri, in northwestern Arkansas and eastern Oklahoma, and in the canyons of the Edwards Plateau in Texas (Sargent 1965).

Cotinus is reported from several stations in Oklahoma (Figure 13). The earliest record of it was made by Nuttall on a trip up the Grand River in 1819. In his journal of July 18 he wrote, "The morning was find and we embarked at sunrise. About 8 o'clock we passed a bend called Eagle's Nest, a mile above which, and its island (sic), a facade of calcareous rock 
appears, inlaid with beds of whitish hornstone. While examining these cliffs, I recognized as new a large shrub, and to my great surprise found it to be a simple-leaved Rhus, scarcely different from Rhus. Cotinus of the south of "Europe and our gardens" (Thwaites 1904-07). In 1928 Palmer reported finding the smoke-tree in a steep, rocky ravine near the base of Rich Mountain in Le Flore County and Little (1942) found it in the Cookson Hills in Cherokee County. Dr. Harriet G. Barclay, of the University of Tulsa collected it in a ravine near Garnett, Rogers County, in 1932. Cotinus has also been collected from a location in Tulsa County approximately three miles east of the present study (Clark 1960). Dr. Albert P. Blair of the University of Tulsa, reports finding it in the vicinity of Spring Creek and Little Spring Creek in Cherokee County. In addition, the author has observed it growing in a canyon five miles west of Claremore, Rogers County.

\section{Ecological Requirements}

The abundance of the disjunct, Cotinus, in this area presents a fascinating picture. The species occurs in large clumps on the upper and lower limestone ledges or on the blocks of limestone that have slumped off the ledge (Figure 6). The largest and most impressive specimens are found on the upper limestone ledge of the north-facing bluff. Here, apparently, its requirements for sunlight and moisture are met most successfully. The roots penetrate the limestone fissures and branch profusely, even when exposed. Many trunks are broken and twisted, but the species has the capacity to produce new shoots, not only from the base, resulting in multiple trunks, but also from the upper branches, making a pollarded effect (Figure 14).

An important relationship between the smoke-tree and the chinquapin oak has become evident in this study. In every location where the author has found the smoke-tree, chinquapin oaks have been present. These two species, unrelated, taxonomically, have become adapted to the same micro-habitat, but they make somewhat different demands upon the environment and fill different niches in the community. The smoke-tree, with the ability of its roots to penetrate the limestone fissures, and its growth habit of proliferating from the base, can gain a foothold on the eroding rock. The chinquapin oak grows so close to the smoke-tree that it overtops the latter although it roots at the base of the limestone ledge. The two species thus seem to complement each other and not to compete. It is noteworthy that a similar relationship exists in Europe where $C$. Coggygria grows in association with another oak (Q. pubescens) and the association is described as the Quercito-Cotinetum forest (Penzes 1958).

Hanson and Churchill (1961) have observed this close association within a community. "The individual plant, in order to live, must establish successful relations with its physical environment as well as with other plants and animals. In nature plants usually grow in groups, not as isolated individuals. These groups may consist of a single species but more often the groups comprise individuals of several species constituting a community.... The kinds of plants that grow in a particular habitat must have the ability to grow not only under the prevailing physical environmental conditions but also in association and competition with neighboring plants."

It could be assumed that since the smoke-tree occurs on limestone it is a calciphilous species. It is possible, however, that the physical character of the rock and the ecological niche of the smoke-tree account for its limestone habitat rather than the chemical properties of the rock. The acidity of the Wann sandstone upland may be a factor, in addition to the dryness, in explaining the almost complete absence of the smoke-tree in the habitat. It should be noted that native specimens of Cotinus already well developed have bee transplanted and grow well in the Tulsa area on sandstone soils if given sufficient water. Such alteration of the usual habitat is thought to be possible only because of its extensive vegetative growth.

Cotinus grows along the limestone ledge on the east slope where the ravine is narrow and sheltered from the drying southerly winds. On the west slope which is more exposed, the clumps are scattered and on the south they are completely absent. It seems evident that the ecological amplitude of the species is small. When exposed to the greater solar radiation and drying winds it is unable to survive. Hanson and Churchill (1961) define ecological amplitude as the "characteristic potentiality for growth of a species with a limited range of environmental conditions". It often determines whether or not a species will be present in a certain habitat or community. 


\section{Paleo-History}

The peculiar distribution pattern of Cotinus obovatus in North America and its isolation from the European species require consideration of its paleo-botanical record. According to Cain (1944), "Major disjunctions seem almost exclusively to have resulted from historical causes which have produced the disjunction in a once more nearly continuous area, through destruction or divergent migrations caused by climatic or some other changes." Since fossil records give the most reliable evidence of paleo-history, it may be noted that the most important fossil members of the Anacardiaceae belong to the genera Rhus, Pistacia, and Anacardites, the latter being a form genus for anacardiaceous foliage of uncertain relationship. Many fossil species of Rhus have been named but not all of them can be considered authentic. Rhus dates from the upper Cretaceous Period, but it seems to have been most prominent in the Oligocene and Miocene (Arnold 1947). According to Barkley (1937) Cotinus is well represented in the Miocene, and Little (1942) traces it as far back as the Cretaceous with Cotinus cretacea Hollick.

In trying to piece together the past history of the Cotinus genus an attempt must be made to trace the evolution of woody angiosperms in North America. Berry (1937) states, "The greatest impediment to a botanical or zoological approach to geologic history is the general lack of realization of the enormous lapse of time involved, and consequently, a complete lack of perspective or orientation." Nevertheless, the challenge is there.

It is the opinion of most authorities that the major part of angiosperm evolution involving the principal modification of flowers took place during the Mesozoic Era. Woody angiosperms appear in abundance in the early part of the cretaceous Period, about 100 million years ago. By the beginning of the Cenozoic Era most of North America, Europe, and Asia were covered by a mixed hardwood forest, the original undifferentiated climax forest. Fossil evidence is sufficient to prove the great extent of this forest, designated by Braun (1950) as the Pan Climax of the Tertiary. It is believed that modern disjuncts of tropical and temperate climates had a continuous range at that time. Later, under the stress of changing climates, new species and ecotypes of woody angiosperms evolved. By the Miocene Period there was undoubtedly a complete separation between the mesophytic forest of North America and Eurasia except for boreal species such as Picea, Salix, Betula, and Acer (Stebbins 1950).

The latter part of the Tertiary saw the rise of great mountain chains in many parts of the world, including the Alps, Himalayas, Western Cordilleras of North America, and the Andes, which brought about the appearance of semi-arid steppes and deserts in the rain shadows of these mountains, greatly restricting the areas occupied by mesophytic plants of both tropical and temperate climates (Stebbens 1950). In North America the forest contracted. There was a gradual shrinkage from west to east due to increased aridity of the interior caused by the rising of the Rocky Mountains and a later retreat of the forest southward due to the climatic fluctuations brought about by the Pleistocene glaciation. The result was a segregation of communities on a basis of moisture requirements and a retreat eastward of those of highest demands (Braun 1935).

If we assume that Cotinus obovatus evolved during the Pan-Climax of the Tertiary it would appear that the species displayed little change while other species were adapting to the diversifications of their environment. Little (1950) states the problem as follows: "Its rarity, discontinuous distribution and lack of related species in the New World, and occurrence as a pioneer species on rocky cliffs all suggest that Cotinus obovatus is an old species formerly of general distribution but now approaching extinction."

It is of interest to consider the record of the close associate of smoke-tree, the chinquapin oak. It is believed that modern members of the Fagaceae were derived during the upper Cretacious Period from the extinct genus Dryophyllum. Quercus is one of the most frequently encountered genera of the upper Cretaceous and Cenozoic. Practically all of the early oaks were of the unlobed or chestnut type. The prominently lobed leaf was rare until the Miocene (Arnold 1947). Since the chinquapin oak is of the chestnut type, it would seem possible that it may be derived from that ancient line.

While Cotinus seems to be past maturity as a genus, its relative, Rhus, has survived and increased its range. The latter genus exhibits wide ecological amplitude and it is represented by over 100 species. It is distributed throughout 
North America from Canada to southern Mexico and from coast to coast. It is also widely distributed in the southern hemisphere and in Eurasia (Sargent 1961). Stebbins (1950) suggests that changing environments have caused the expansion and variation of some groups, the decline and extinction of others and have left some groups relatively unchanged. The ability of a group to respond to environmental change depends on certain biological characteristics of the group.

\section{Present Status}

It seems consistent with the above considerations to call the American smoke-tree a relict, since its present survival appears to be but a remnant of past distribution and importance. In ecological terms, according to Weaver and Clements (1938), a relict is a species or community which has remained after some change has resulted in the elimination or modification of an earlier vegetation, often a climax. "Vegetational analysis of relict communities combines observation, scrutiny of scientific reports and records, experimentation, and interpretation; but its essential feature is the search for areas continuously protected against disturbance." Because of their rugged topography, Cotinus habitats have been relatively undisturbed by man. In this particular study, the land is of little economic importance due to its irregularity and the impurity of the limestone. According to the present owner of the study area, it has not been used except to pasture a few cattle from time to time. It is believed that Indians, particularly the Osages, camped on these bluffs before the coming of the white man, for Indian arrowheads and artifacts have been found.

\section{SUMMARY}

A high upland and bluff above the Arkansas River, encompassing different rock strata and slope exposures, was studied to determine the ecological factors affecting plant distribution. Here the Wann sandstone caprock overlies the lola formation, well exposed on the north bluff, and consisting of upper and lower limestone members interbedded with shale. The soil on the upland contrasts with that of the slopes in $\mathrm{pH}$ and quantity of organic matter.

A complete collection of plants was made throughout the area during an entire growing season, and is entered in the University of Tulsa Herbarium.

A small percentage of the herbaceous species is definitely related to the underlying strata. The woody vegetation was analyzed by belt transects which crossed the different strata on all slope exposures. The dominants on the upland are post oak, with an Importance Percentage of 65.9 , and blackjack oak, with an I.P. of 41.4. The dominants on the limestone are chinquapin oak, with an I.P. of 34.9 , and Shumard's oak, with an I.P. of 34.9 , with the former confined largely to the upper limestone, and the latter to the base of the lower limestone outcrop. The transects on the different slope exposures showed marked changes in species populations and in degree of mesophytism.

A significant tree on the limestone is the American smoke-tree, Cotinus obovatus, a comparatively rare species in Oklahoma. Its multipletrunked growth form and its freely branching root system adapt it to the eroding limestone ledges. It grows in close association with chinquapin oak, the two species unrelated taxonomically, but occupying different niches in the same microhabitat. It is notable that the European smoke-tree is also closely associated with an oak. The disjunct distribution of the American smoke-tree indicates that it was probably widespread in former geologic periods. Its survival as a relict with limited ecological amplitude is due to the continuance of the microhabitats to which it is so well adapted.

\section{ACKNOWLEDGEMENTS}

Grateful acknowledgements are due to the many, who have been of assistance in the preparation of this thesis - most especially to Dr. Harriet G. Barclay for direction of the study as well as her inspiration and encouragement, to Dr. Paul Buck who has helped in many ways, and to Mr. and Mrs. A.W. Swift who so generously gave permission for the use of their property for this study, to Miss Sandra Forehand, a frequent companion on "collection" trips, and to Mrs. Jean Hamilton for her help in the collection and identification of grasses. To my husband, Walter, whose patience has been unflagging, I express my deepest thanks. 


\section{LITERATURE CITED}

Arnold, Chester A. 1947. An introduction to Paleobotany. New York and London, McGraw Hill Book Company, Inc.

Barclay, Harriet G. 1947. The woody vegetation of Bear's Glen, a Washington Irving stopover. Proc. Okla. Acad. Sci. 28: 39-57.

Barkley, Fred A. 1937. A monographic study of Rhus and its immediate allies in North and Central America, including the West Indies. Annals of the Missouri Botanical Garden 24: 300-303.

Berry, E. W. 1937. Tertiary floras of eastern North America. Botanical Review 3: 31-46.

Braun, E. Lucy 1935. The undifferentiated deciduous forest climax and the association segregate. Ecology 16 : 514-519 1967. Deciduous Forests of Eastern North America. New York and London, Hafner Publishing Company.

Brizicky, George K. 1962. The genera of Anacardiaceae in the southeastern United States. Journal of Arnold Arboretum Vol. XLIII No. 14, pp. 366-68.

Bruner, W. E. 1931. The vegetation of Oklahoma. Ecol. Monogr. 1: 99-188.

Cain, Stanley A. 1944. Foundations of Plant Geography. New York and London, Harper and Brothers.

Chickshev, A. G. ed. 1961. Plant Indicators of Soils, Rocks, and Subsurface Waters. Conference on Indicator Geobotany. Moscow. Authorized translation from Russian. New York, Consultants Bureau, 1965.

Clark, Maxine. 1960. Interesting plant collections from Tulsa County. Proc. Okla. Acad. Sci. 40: 7-8.

Hanson, Herbert C., and Churchill, Ethan D. 1961. The Plant Community. New York, Reinhold Publishing Corp.

Little, Elbert L., Jr. 1942. American smoke-tree (Cotinus obovatus Raf.), one of Oklahoma's rarest tree species. Proc.
Okla. Acad. Sci. 23: 21-23.

Oakes, Malcolm. 1952. The geology and mineral resources of Tulsa County Oklahoma. Oklahoma Geological Survey Bulletin No. 69.

Penzes, Dr. A. 1958. Data to the ecology and taxonomy of the Cotinus genus. Acta Botanica Sinica 7: 167-169.

Phillips, E. A. 1959. Methods of Vegetation Study. New York, Henry Holt and Co., Inc.

Rice, E.L., and Wm. T. Penfound. 1959. The upland forests of Oklahoma. Ecology 40: 593-608.

Sargent, Charles Sprague. 1961. Manual of Trees of North America, Vol. 2. New York, Dover Publications, Inc.

Stebbins, G. Ledyard, Jr. 1950. Variation and Evolution in Plants. New York, Columbia University Press.

Thwaites, Reuben Gold 1853-1913, ed. 1904-07. Early Western Travels 1748-1846, Vol. 13. Cleveland, O., The A.H. Clark Company.

Weaver, John E. and Clements, Frederic E. 1938. Plant Ecology. New York and London, McGraw Hill Book Company, Inc.

Waterfall, U. T. 1966. Keys to the Flora of Oklahoma, 3rd. ed. Stillwater: Privately published.

Yearbook of Agriculture. 1955. Water. Washington, D. C., U. S. Govt. Printing Office.

Editor's notes:

As a charter member of Oklahoma Native Plant Society, Anne Long was an influential figure in its organization. It is with honor that we present her master's thesis as an important historical study to be used by future researchers, teachers, and leaders for native plant conservation and education.

While time has misplaced the original sources for several of her figures, modern technology has provided updated and visually improved ones as noted. [SAS] 


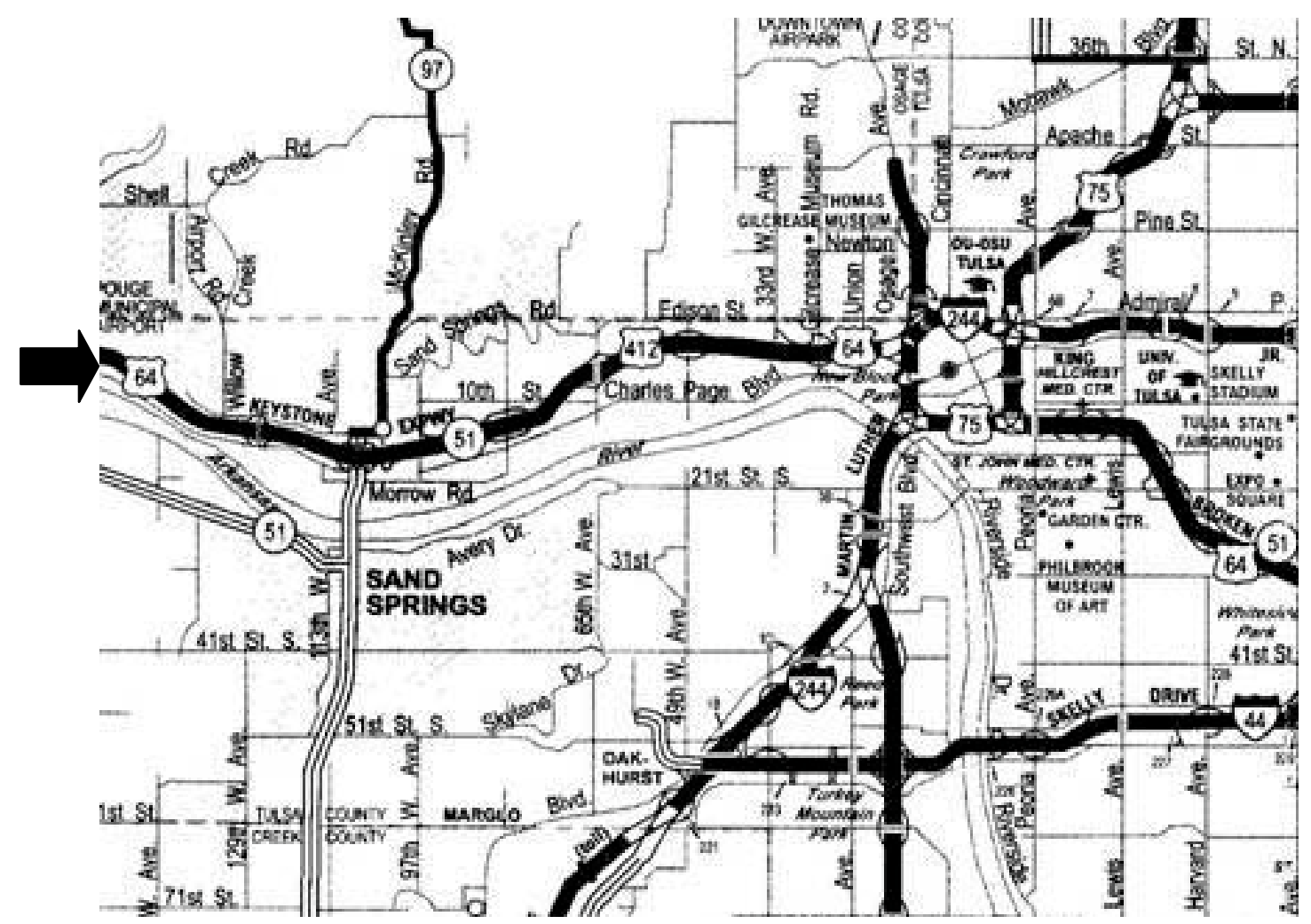

Figure 1 Arrow indicates location of study site with reference to Tulsa County and the Arkansas River. [Map substitution courtesy of the Oklahoma Department of Transportation.]

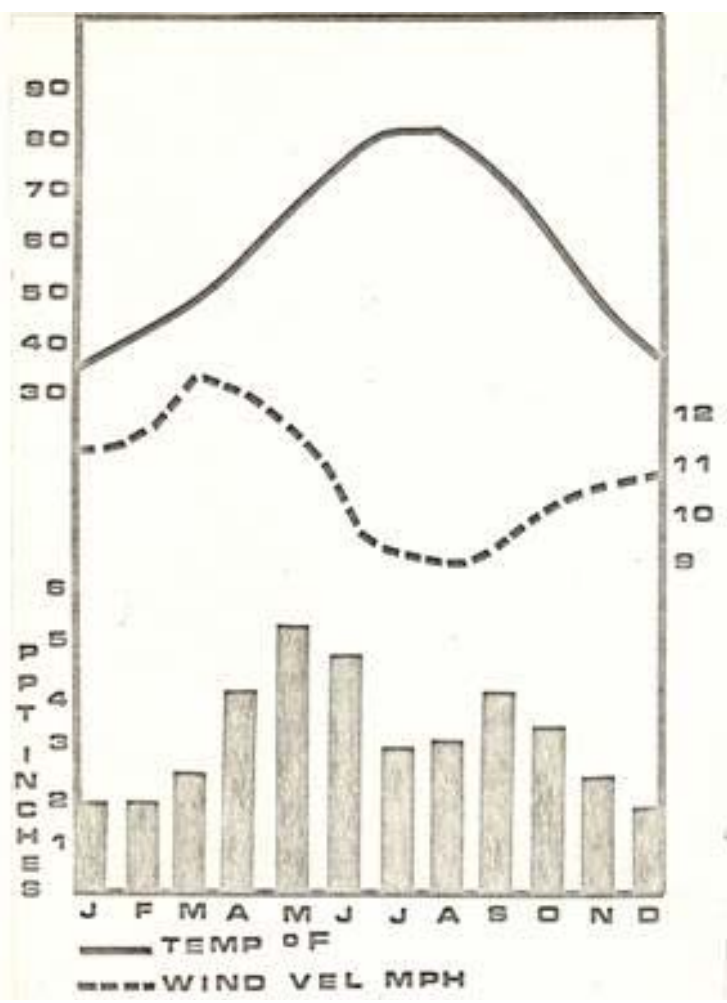

Figure 2 Average monthly temperature, precipitation, and wind data for Tulsa County. From U.S. Weather Bureau (1931-68). 


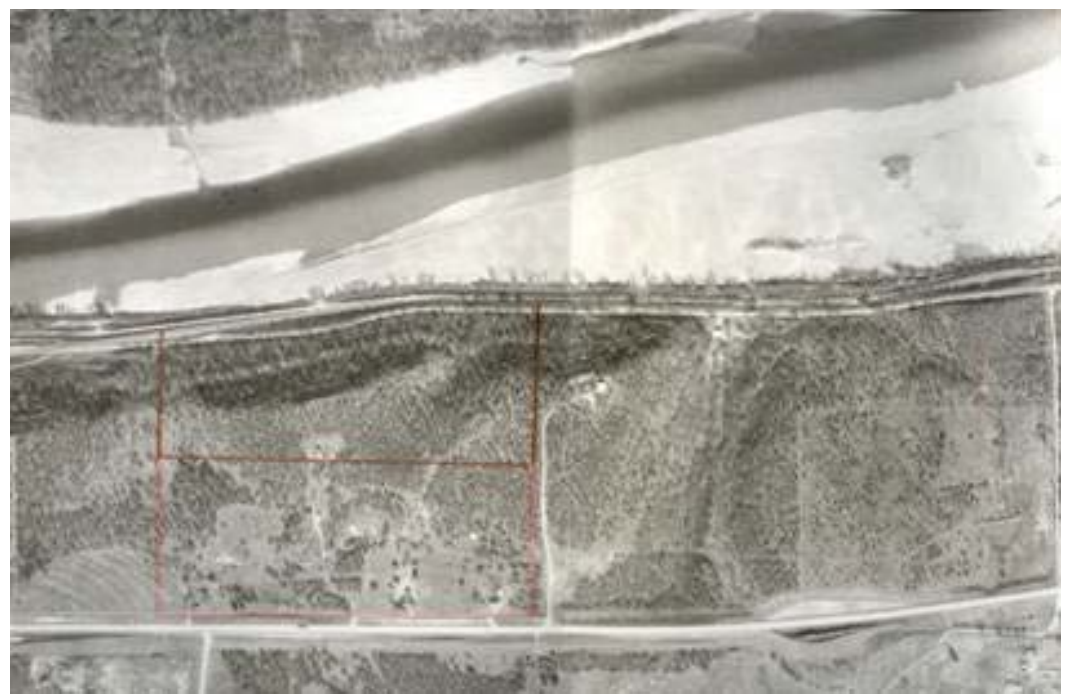

Figure 3 Aerial photograph of S $1 / 2$ of section 11, T.19 N., R.10 E., Tulsa County. By Aerial Photo Service 2669. Scale: $1 "=1000$ '. [Study site border appended for comparison to contour map.]

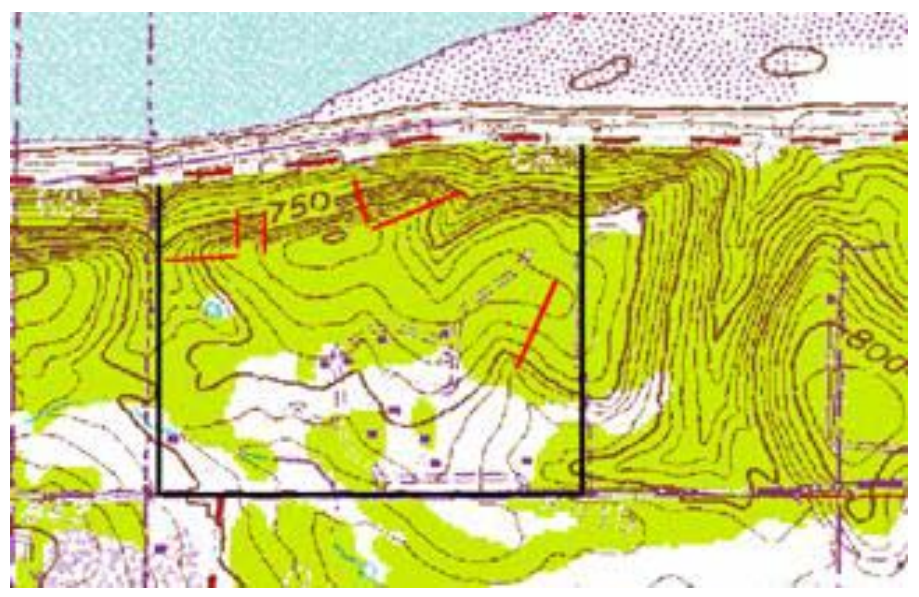

Figure 4 Contour map of study site and environs. Study site border and transects indicated. Contour Interval = 10' Scale 1" = 1000'. [Geo Information Systems, University of Oklahoma (www.geo.ou.edu), substituted with permission.]

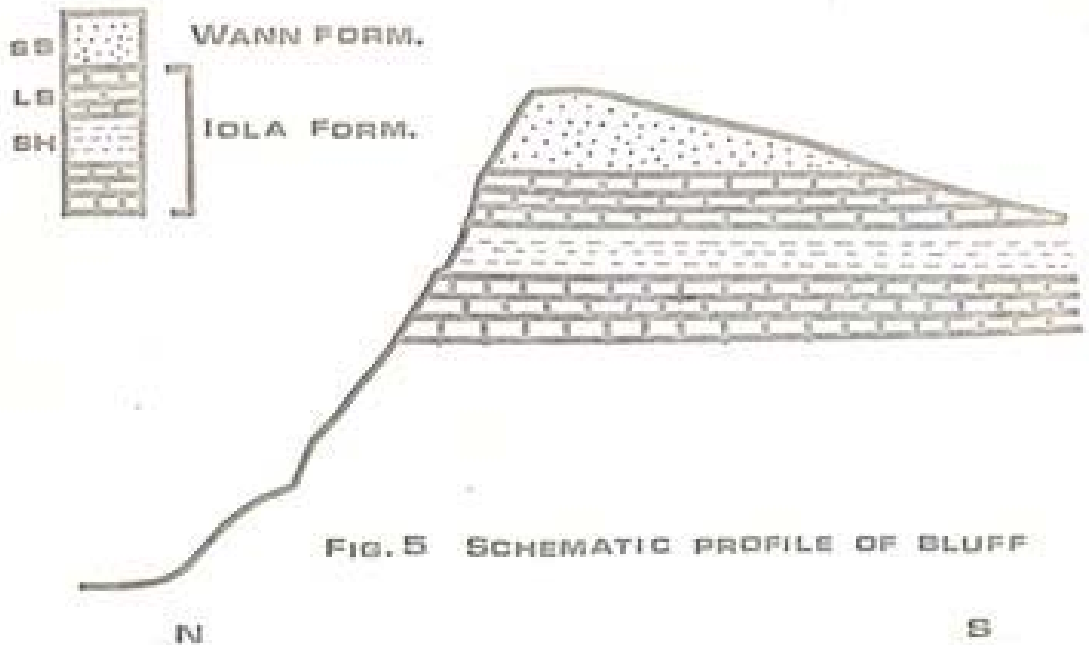

Figure 5 Schematic profile of bluff

Long, A.W. 


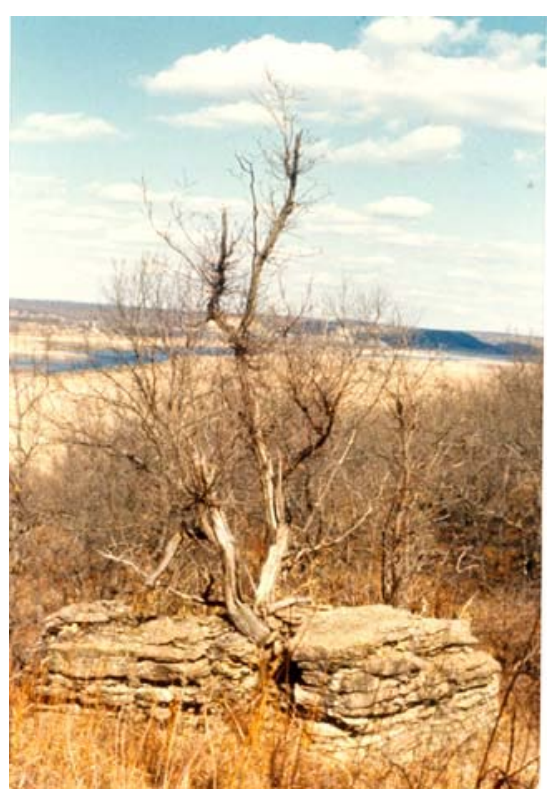

Figure 6 Isolated slump block of lola limestone showing smoke-tree.

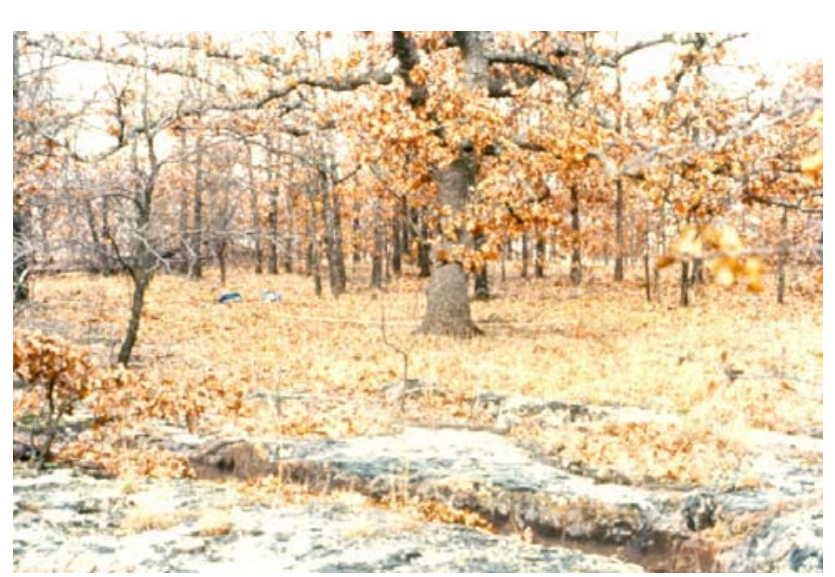

Figure 7 Wann sandstone caprock.

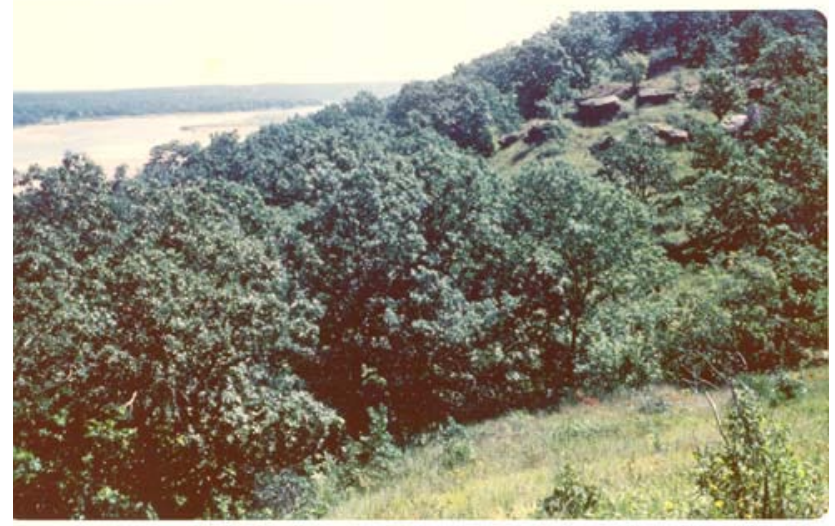

Figure 8 Dense tree growth on the north-facing bluff.

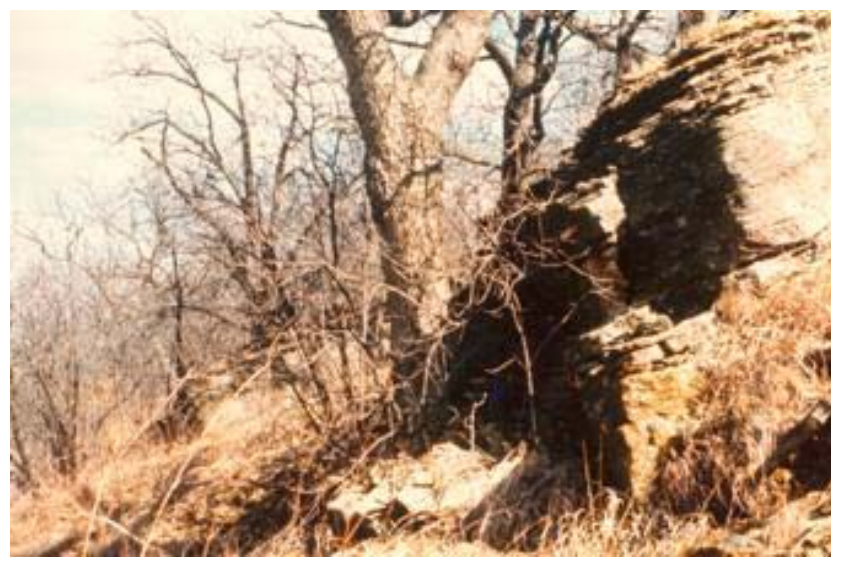

Figure 9 Upper ledge of lola limestone, with smoke-tree and large chinquapin oak.

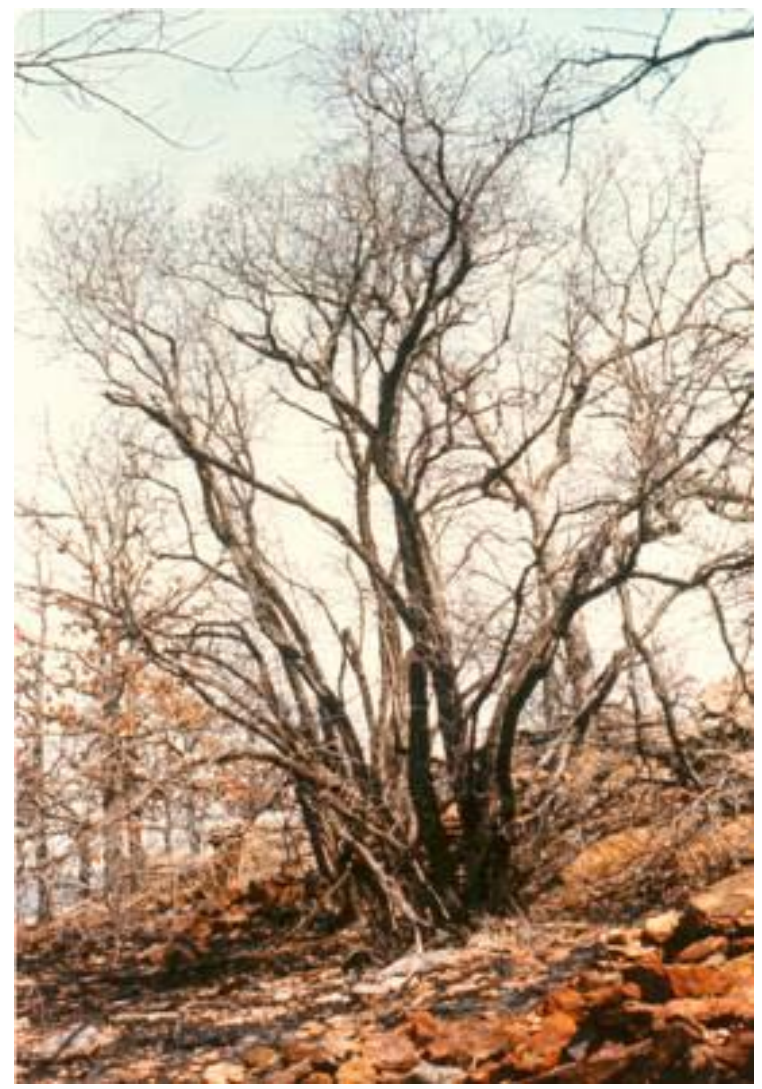

Figure 10 Multiple-trunked smoke-tree on limestone. 

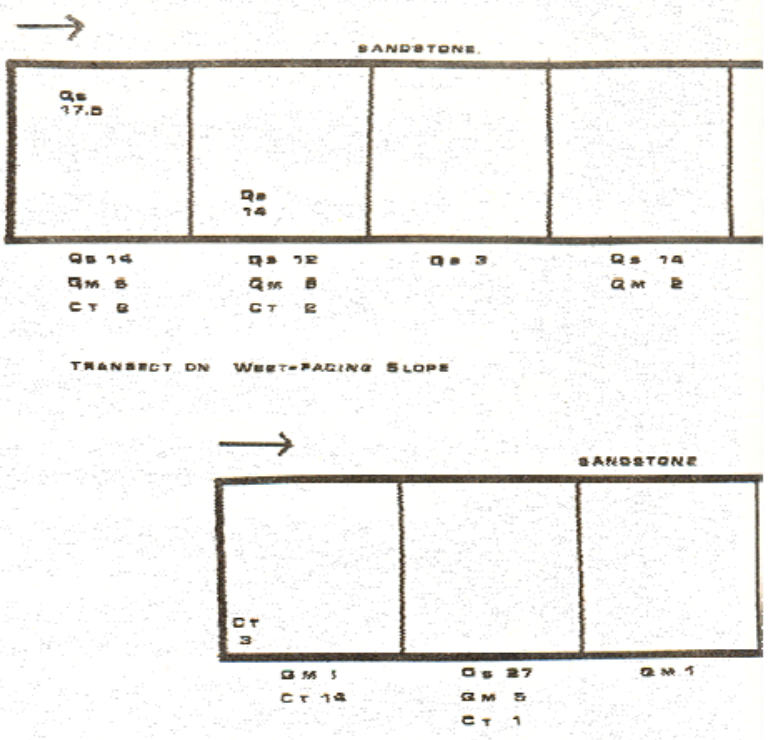

CMATY OF TAANBECTA

BCACE: EACM ERUARI $5 \mathrm{~m}, \times 5 \mathrm{M}$

LMOND:

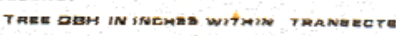

RERROAUCSION COUNTE BELOW TMANERET

Co cotinue oBovatis

CT CaRYa TEXane.

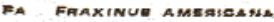

CSK RUEREU MARHANDIE

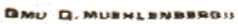

ae a. atELLATA

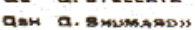

$\longrightarrow$ INGICATHE DOWNELGEE

TRANBECT ON NOETH-FAEINE BLUFF IWESTM

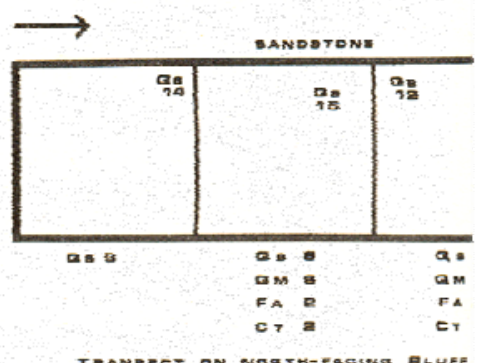

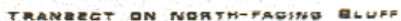

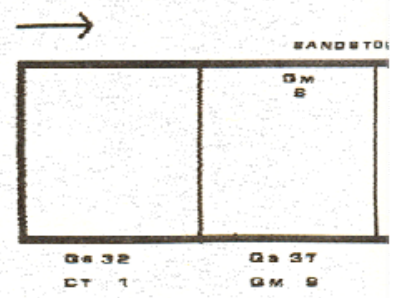

TRANBEDT ON NOMTM-FACINO

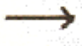

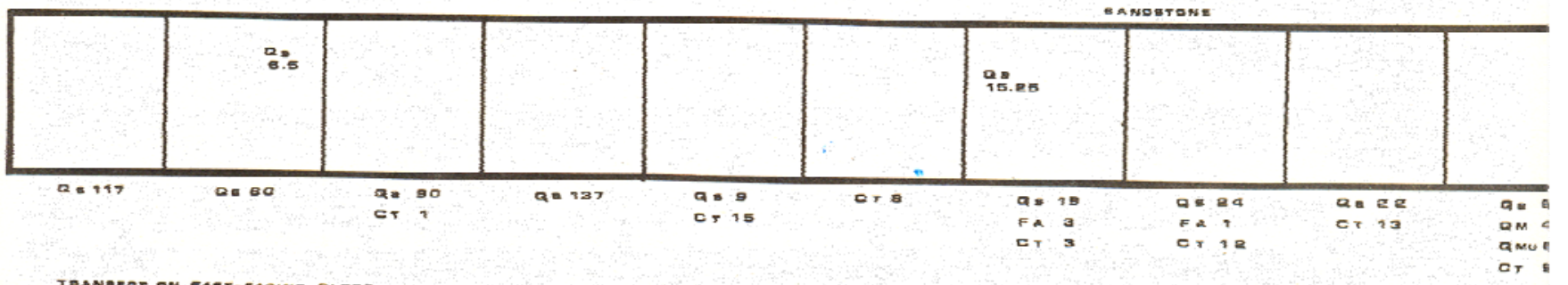

Figure 11 Chart of transects.

Long, A.W. 

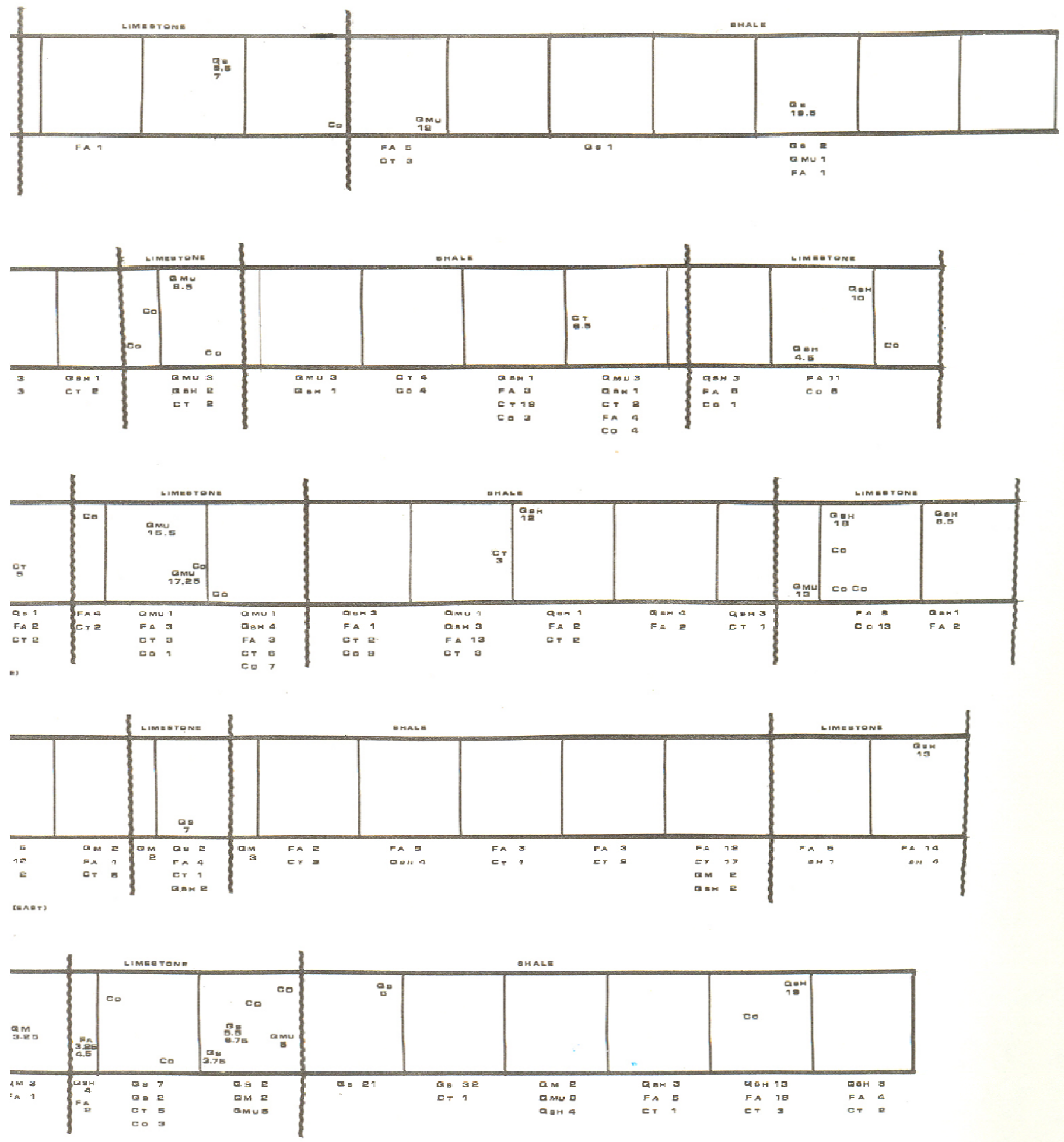


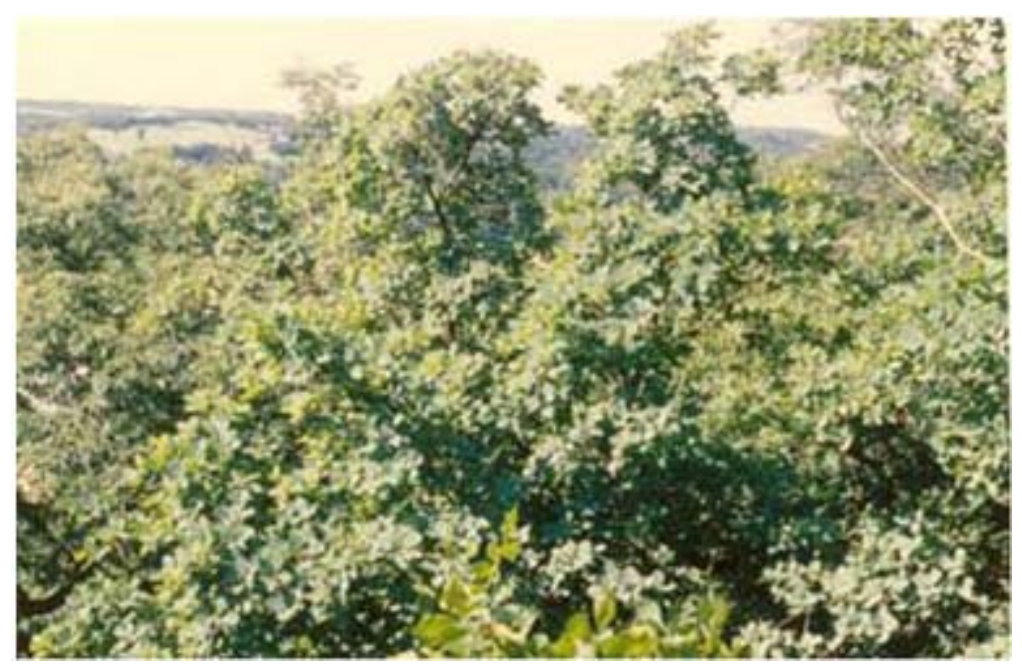

Figure 12 Summer foliage of smoke-tree.

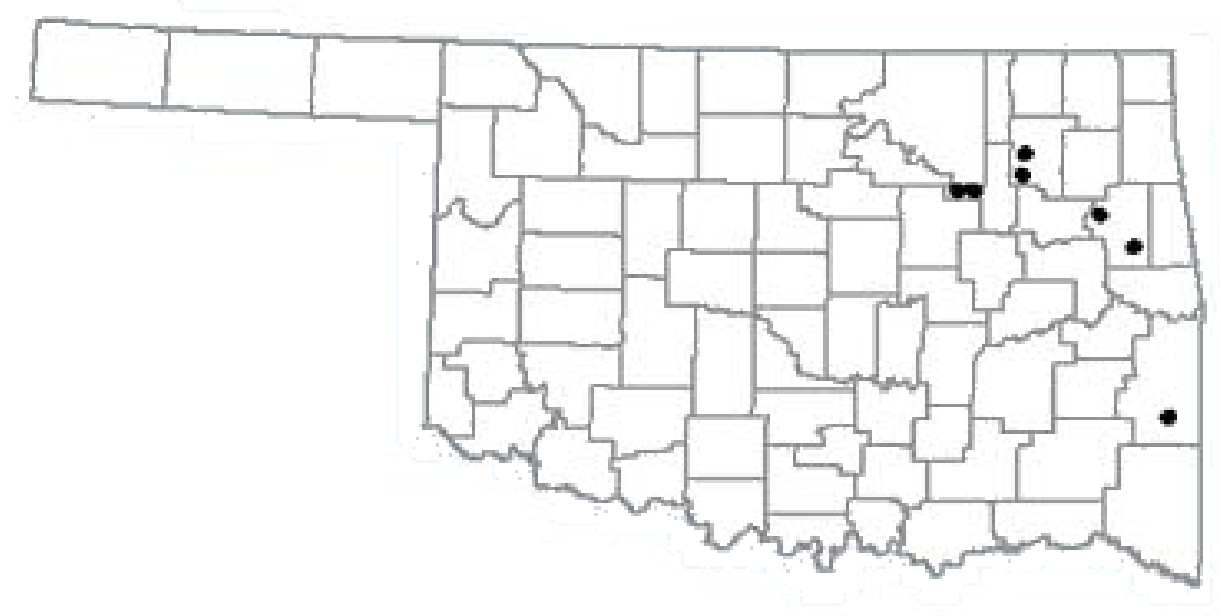

Figure 13 Distribution of Smoke-tree in Oklahoma. [State map substitution courtesy of Geo Information Systems, University of Oklahoma @geo.ou.edu.]

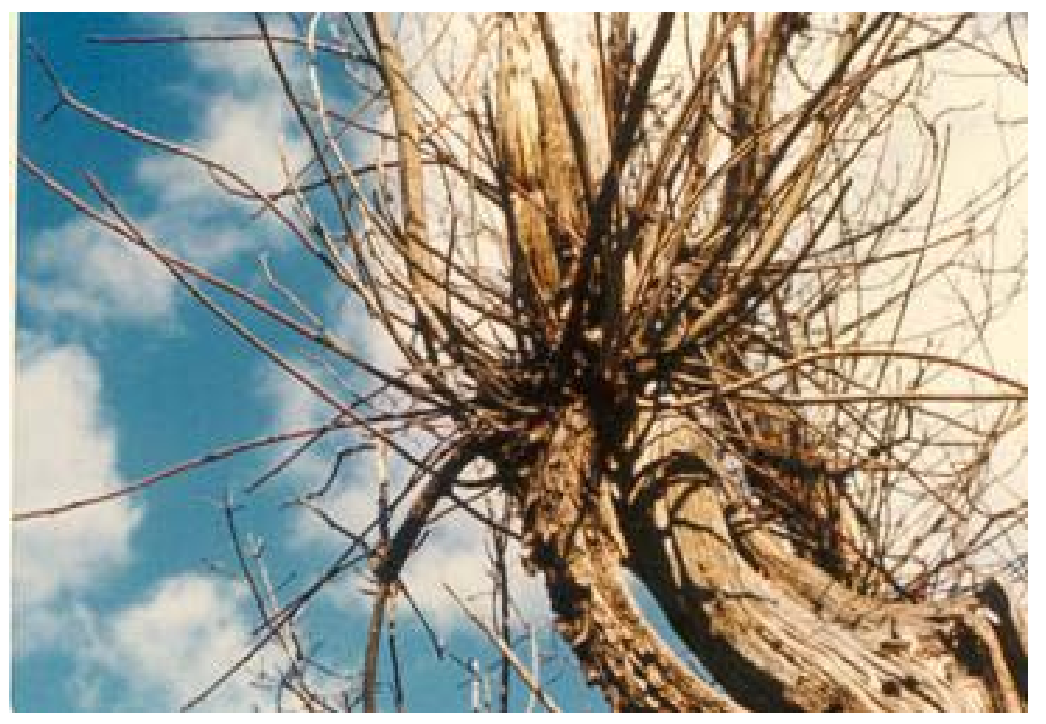

Figure 14 New shoots of Cotinus from upper branches.

Long, A.W. 


\section{TABLE 1 COMPLETE COLLECTION OF VASCULAR PLANTS MADE OVER THE GROWING SEASONS OF 1967-8 AT THE STUDY AREA, LISTED BY FAMILIES.}

NOMENCLATURE ACCORDING TO U.T. WATERFALL (1966). Editor's note: Species nomenclature has been updated according to Kartesz (1994) and USDA-NRCS (16 June 2004). Asterisks denote name changes;

revisions follow in brackets. [PAF]

Ophioglossaceae

Ophioglossum engelmannii Prantl.

Polypodiaceae

${ }^{*}$ Notholaena dealbata (Pursh) Kunze

[Argyrochosma dealbata (Pursh.)

Windham]

Pellaea atropurpurea (L.) Link, var. atropurpurea

Woodsia obtusa (Spreng.) Torr.

*Gramineae [Poaceae]

Andropogon gerardii (Vitman, var. gerardi

*Andropogon saccharoides Sw.

[Bothriochloa laguroides (Sw.) Rydb.]

${ }^{*}$ Andropogon scoparis Michx.

[Schizachrium scoparium (Michx.) Nash

Aristida purpurescens Poir.

Bouteloua curtipendula (Michx.) Torr.

Digitaria filiformis (L.) Koel., var. filiformis

Elymus virginicus L., var. virginicus forma virginicus

Eragostris capillaris (L.) Nees

Eragrostis hirsuta (Michx.) Nees

Eragrostis intermedia Hitchc.

${ }^{*}$ Eragrostis oxylepis (Torr.) Torr., var. oxylepis

[Eragrostis secundiflora J. Presl. ssp oxylepis (Torr.) Koch]

Eragrostis spectabilis (Pursh) Steud., var. sparsihirsuta Farwell

Gymnopogon ambiguus (Michx.) B.S.P.

Muhlenbergia sobolifera (Muhl.) Trin.

Panicum capillare L., var. capillara

${ }^{*}$ Panicum lanuginosum Ell., var. fasciculatum (Torr.) Fern.

[Dicanthelium acuminatum (Sw.) Gould \& C.A. Clark, var. fasciculatum (Torr.) Freckmann

*Panicum oligosanthes Schultes, var. Scribnerianum (Nash) Fern.

[Dicanthelium oligosanthes (J.A. Schultes) Gould, var. scribnerianum (Nash) Gould.]

Panicum virgatum $\mathrm{L}$.

*Setaria geniculata (Lam.) Beauv.

[Setaria parviflora (Poir.) Kegguelen

Sorghastrum nutans (L.) Nash

Sporobolus clandestinus (Biehler) Hitchc.

Commelianaceae

Commelina erecta L. var. erecta, forma intercursa Fern.

Tradescantia ohiensis Raf., forma pilosa Waterfall

Liliaceae

Allium perdulce S. V. Fraser

Camassia angusta (Engelm. \& Gray)

Blankenship

Nothoscordum bivalve (L.) Britton

Smilax bona-nox L.

Smilax glauca Walt., var. glauca

Yucca arkansana Trel., var. arkansana

Iridaceae

Sisyrinchium campestre Bickn., forma campestre

Sisyrinchium campestre Bickn., var. kansanum (Bickn.)

Steym.

Orchidaceae

Spiranthes cernua (L.) Richards

Juglandaceae

Carya cordiformis (Wang) K. Koch

Carya texana Buckl. (C. Buckleyi Durand)

Fagaceae

Quercus marilandica Muench.

Quercus muehlendbergii Englem., var. muehlenbergii

Quercus Shumardii Buckl., var. schneckii (Britton) Sarg.

Quercus stellata Wang.

Ulmaceae

Celtis laevigata Willd.

Ulmus americana L.

Ulmus rubra Muhl. (U. fulva Muhl.)

Moraceae

Morus rubra L.

Nyctaginaceae

Mirabilis albida (Walt.) *MacM. [Heimerl.]

Portulacaceae

Claytonia virginica $\mathrm{L}$.

*Portulaca mundula Johnston

[Portulaca pilosa L.]

Talinum parviflorum Nutt.

Polygonaceae

Polygonum tenue Michx.

Ranunculaceae

${ }^{*}$ Delphinium virescens Nutt.

[Delphinium carolinianum Walt., var. virescens (Nutt.) Brooks] 
Capparidaceae

Cleome serrulata Pursh.

Cruciferae

Draba cuneifolia Nutt., var. cuneifolia

Draba reptans (Lam.) Fern., var. reptans

Lepidium virginicum $\mathrm{L}$.

Crassulaceae

Sedum Nuttallianum Raf.

Saxifragaceae

${ }^{*}$ Ribes odoratum Dougl.

[Ribes aureum Pursh, var. villosum DC.]

Rosaceae

Agrimonia rostellata Wallr.

Amelanchier arborea (Michx. f.) Fern.

Crataegus sp.

Prunus americana Marsh.

Prunus angustifolia Marsh.

Rosa carolina L.

Rosa setigera Michx., var. tomentosa T. \& G.

Leguminosae

Acacia angustissima (Mill.) Kunze, var. hirta (Nutt.) Robinson

Amorpha canescens Pursh., forma canescens

Amorpha fruticosa $\mathrm{L}$.

Amphicarpa bracteata (L.) Fern., var. bracteata

Astragalus crassicarpus Nutt., var. crassicarpus

${ }^{*}$ Baptisia leucophaea Nutt. var. leucophaea

[Baptisia bracteata (Nutt.) Kartesz \& Ghandi var. leucophaea Muhl.ex. Ell.]

${ }^{*}$ Cassia fasciculata Michx.

[Chamaecrista fasciculata (Michx.) Greene]

${ }^{*}$ Cassia nictitans L.

[Chamaecrista nictitans (L.) Moench]

Cercis canadensis L., var. canadensis

Clitoria mariana L.

Dalea purpurea Vent., sen. lat.

Desmanthus illinoiensis (Michx.) MacM.

Desmodium canescens (L.) DC Wood

Desmodium glutinosum (Muhl.) Wood

Desmodium sessilifolium (Torr.) T. \& G.

Galactia volubilis (L.) Britton, var. mississippiensis

Lespedeza violacea (L.) (Pers.)

Lespedeza stuevei Nutt., forma stuevei

${ }^{*}$ Psoralea tenuiflora Pursh.

[Psoralidium tenuiflora (Pursh) Rydb.]

Stylosanthes biflora (L.) BSP., var. hispidissima (Michx.)
Pollard \& Ball

Tephrosia virgjniana (L.) Pers., var. virginiana

Linaceae

Linum sulcatum Riddell

Oxalidaceae

Oxalis corniculata L.

Oxalis violacea L., var violaceae

Geraniaceae

Geranium carolinianum L.

Euphorbiaceae

Acalypha virginica $\mathrm{L}$.

Croton glandulosus L., var. septentrionalis Muell. Arg.

Euphorbia corollata L. var. corollata

Euphorbia hexagona Nutt.

Euphorbia spathulata Lam.

Tragia urticifolia Michx.

Anacardiaceae

Cotnius obovatus Raf.

Rhus aromatica Ait., var. aromatica

Rhus copallinum L., var. latifolia Engl.

Rhus glabra L.

${ }^{*}$ Rhus Toxicodendron $\mathrm{L}$.

[Toxicodendron pubescens P. Mill.]

Aquifoliaceae

Ilex decidua Walt.

Celastsraceae

Celastrus scandens $\mathrm{L}$.

Rhamnaceae

Ceanothus americanus L., var. Pitcheri T. \& G.

Vitaceae

Vitis aestivalis Michx.

Malvaceae

Callirhoe alcaeoidesi (Michx.) Gray

*Sphaeralcea angusta (Gray) Fern.

[Malvastrum hispidum (Pursh) Hochr.

Guttiferae*

[Hypericaceae]

Hypericum punctatum Lam.

Cistaceae

Lechea tenuifolia Michx.

Violaceae

Viola pensylvanica Michx., var. leiocarpa (Fern. \& Wieg.) Fern.

Lythraceae

${ }^{*}$ Cuphea petiolata (L.) Koehne

[Cuphea viscosissima Jacq.]

Onagraceae

Oenothera laciniata Hill, var. laciniata

Oenothera linifolia Nutt.

Oenothera speciosa Nutt.

Umbelliferae

Daucus pusillus Michx.

Long, A.W. 
Spermolepis echinata (Nutt.) Heller Torilis japonica Houtt. DC

\section{Cornaceae}

Cornus drummondii Meyer

Ebenaceae

Diospyros virginiana L., var. virginiana

Oleaceae

Fraxinus americana L., var. americana

Gentianaceae

Sabatia campestris Nut., forma campestris

Apocynaceae

Apocynum cannabium $\mathrm{L}$., var. pubescens (Mitchell) A. DC.

Asclepiadaceae

Asclepias tuberosa L.

Asclepias verticillata $\mathrm{L}$.

Asclepias viridis Walt.

Asclepias viridiflora Raf., var. viridiflora

Boraginaceae

Heliotropium tenellum (Nutt.) Torr.

Myosotis verna Nutt.

Onosmodium hispidissium Mack.

Verbenaceae

* Lippia incisa (Small) Tidestrom

[Phyla nodiflora (L.) Greene]

${ }^{*}$ Verbena canadensis (L.) Britt.

[Glandularia canadensis (L.) Nutt.]

Verbena stricta Vent., forma stricta

*Labiatae [Lamiaceae]

Monarda fistulosa L., var. fistulosa

Salvia azurea Moench., var. grandiflora Benth.

Scutellaria parvula Michx. var. parvula

Teucrium canadense L., var. virginicum (L.) Eat.

Scrophulariaceae

${ }^{*}$ Gerardia heterophylla Nutt.

[Agalinus heterophylla (Nutt.) Small]

*Gerardia tenuifolia Vahl., var. parviflora Nutt.

[Agalinus tenuifolia Vahl, Raf. var. parviflora Nutt. Pennell]

Penstemon cobaea Nutt.

Penstemon tubiflorus Nutt.

*Linaria canadensis (L.) Dumont, var. texana (Scheele) Pennell

[Nuttalanthus canadensis (L.) D.A. Sutton, var. texana (Scheele)Sutton]

Solanaceae

Physalis pubescens L., var. integrifolia (Dunal) Waterfall

*Solanum Torreyi Gray, forma Torreyi

[Solanum dimidiatum Raf.]

Acanthaceae

Ruellia humilis Nutt.
Plantaginaceae

Plantago Purshii R. \& S., var. Purshii

Plantago virginica $L$.

Rubiaceae

${ }^{*}$ Diodia teres Walt., var. setifera Fern. \& Grisc. [var. teres]

Gallium aparine L.

Hedyotis nigricans (Lam.) Fosb.

Caprifoliaceae

Symphoricarpos orbiculatus Moench

Triosteum perfoliatum $\mathrm{L}$.

Virburnum prunifolium L., var. ferrugineum Torr. \& Gray

Campanulaceae

${ }^{*}$ Specularia biflora (R. \& P.)

Fisch. \& Mey.

[Triodanis perfoliata, var. biflora

(R. \& P.) Bradley]

*Specularia leptocarpa (Nutt.) Gray

[Triodanis leptocarpa (Nutt.) Nieuwl.]

*Specularia perfoliata (L.) A. DC.

[Triodanis perfoliata (L.) Nieuwl.]

*Compositae [Asteracae]

${ }^{*}$ Achillea lanulosa Nutt., forma lanulosa

[Achillea millefolium L.]

Ambrosia artemisiifolia L.

*Antennaria plantaginifolia (L.) Richards

[Antennaria parlinii Fern]

${ }^{*}$ Aster azureus Lindl.

[Aster oolentangiensis Riddell]

Aster ericoides L. (A. multiflorus)

Aster patens Ait., var. patentissimus (Lindl.) T. \& G.

*Aster sagittifolius Wedemeyer

[Aster cordifolius L., var. sagittifolius (Wed. ex Willd.) A.G. Jones]

Astranthium integrifolium (Michx.)Nutt.

${ }^{*}$ Cacalia plantaginea (Raf.) Shinners

[Arnoglossum plantigineum Raf.]

Chrysopsis pilosa Nutt.

Cirsium altissimum (L.) Hill.

Erigeron strigosus Muhl. Ex. Willd.

Erigeron philadelphicus L.

Eupatorium altissimum L.

Gnaphalium obtusifolium L.

* Gutierrezia dracunculoides (DC) Blake

[Amphiachris drancunculoides (DC) Nuttal]

Helianthus hirsutus Raf., var. trachyphyllus T. \& G.

*Kuhnia eupatorioides L., var. corymbulosa T. \& G.

[Brickellia eupatorioides L., var. corymbulosa (T. \& G.) Shinners]

Liatris punctata Hook,

var. nebraskensis Gaiser

Liatris squarrosa (L.) Michx., 
var. hirsuta Rydb. Gaiser

*Pyrrhopappus scaposus DC.

[Pyrrhopappus grandiflorus (Nutt.)]

Ratibida columnifera (Nutt.) W. \& S.
Rudbeckia hirta L.

Solidago missouriensis Nutt., var. fasciculata Holz.

Vernonia baldwinii Torr., var. Baldwinii

\begin{tabular}{|c|c|c|c|c|c|c|c|c|c|c|c|c|}
\hline & \multicolumn{4}{|c|}{ Sand } & \multicolumn{4}{|c|}{ IInstane: } & \multicolumn{4}{|c|}{ single } \\
\hline & $\mathbb{E}$ & 5 & 12 & Are & c & 5 & $\mathrm{H}$ & AM & c & 5 & M & Are. \\
\hline $\mathrm{pH}$ & 4.9 & 4,1 & 5.9 & 5.3 & 7.2 & 7.0 & 5.9 & 7.0 & 7.5 & 7.7 & 7.8 & 7.7 \\
\hline$\left[\begin{array}{l}\text { Organic } \\
\text { mattert }\end{array}\right.$ & 28. & 35 & 34 & 32.3 & 46 & 50 & 34 & 43.3 & 70 & 10 & 51 & 63.7 \\
\hline
\end{tabular}

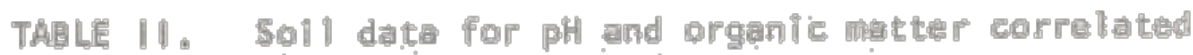

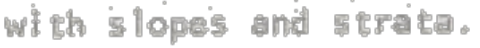

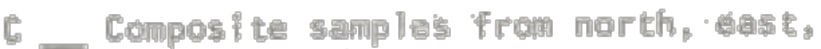
and west shopes.

\$ - Samples from south stope.

N - Sanples from morth sloper.

Ane 


\begin{tabular}{|c|c|c|c|c|c|c|c|c|c|c|c|c|c|c|}
\hline & \multicolumn{7}{|c|}{ gannsTo: } & \multicolumn{7}{|c|}{ dunate } \\
\hline & 6en: & 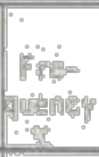 & 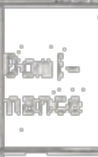 & 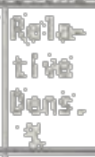 & 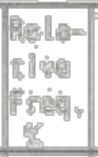 & 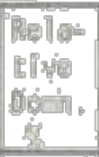 & 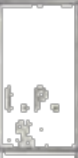 & 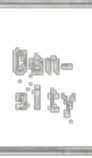 & 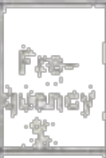 & Dathe & 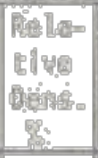 & 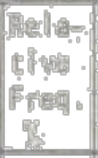 & 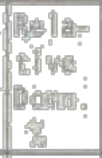 & 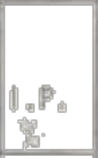 \\
\hline 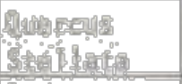 & 盛 & 核 & 19. & Pix 5 & 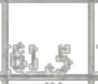 & 28. & 20.6 & .27 & 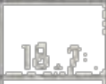 & Lis:? & 3 : & 29 & 9 & 5 \\
\hline Hequed & กต่ & 10.3 & 黑蹈: & 23. & $22^{3}$ & 9 & 106.3 & & & & & & & \\
\hline 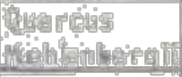 & & & & & & & & 舟最 & 25 & 39: & 27.7 & 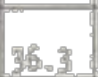 & Hen & 3h.9.9. \\
\hline 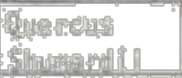 & & & & & & & & . 31 & 25 & 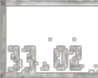 & 27.7 & 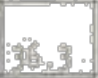 & Hin. & 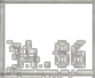 \\
\hline 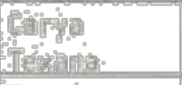 & -97 & 2al & 95 & 115,2 & 115.9 & $2.2:$ & 里, & & & & & & & \\
\hline 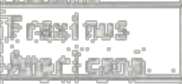 & & & & & & & & 125 & . & 1.5 & 11 & 9 & 1. & 7.3 \\
\hline 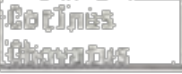 & & & & & & & & 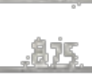 & 50 & & 43.8 & BR. & & \\
\hline
\end{tabular}

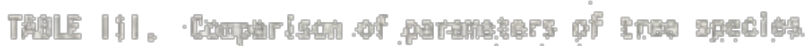

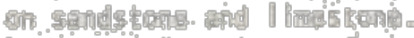

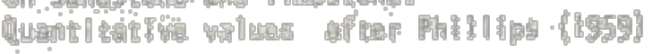




\section{Cotinus obovatus Raf. (Smoke-tree) in Oklahoma Bruce W. Hoagland \\ Oklahoma Biological Survey and Department of Geography University of Oklahoma, Norman, OK 73019}

Cotinus obovatus is a shrub or small tree, up to $6.5 \mathrm{~m}$ (20 feet) tall. The twigs are orange to brown, glabrous (without hairs), and aromatic when crushed (Figure 1). The sap is resinous and strong-smelling. The wood is yellow to orange in color with creamy colored sapwood. The leaves are alternate, simple, elliptical to obovate, $5-13 \mathrm{~cm}$ (2-5 inches) long, and 4-7.5 cm (1.6-3 inches) wide. They are pointed at the base, but rounded to weakly pointed at the apex and margins are entire. Leaves turn orange to scarlet in the fall (Figure 2). Flowers bloom in early spring, are very small, and have five petals and five sepals that are greenish in color, with five stamens and one pistil. Separate male and female flowers are present on the same plant. The wispy panicles measure 15 $\mathrm{cm}$ (6 inches) or more in length and are the root of the common name smoke-tree (Figure 3). However, there are few flowers in the panicle and many of them are sterile. Fruits are small drupes about 5 $\mathrm{mm}$ (0.2 in) in diameter. Some flowers are sterile and their stalks are long and covered with purplish or brownish hairs. The tree sprouts readily from the roots (Elias 1987, Hightshoe 1988, Kurz 1997, Little 1996, Sargent 1905).

Cotinus species are members of the Anacardiaceae (cashew) family. Other members of this family occurring in Oklahoma include Rhus aromatica (skunkbrush), Rhus copallinum (winged sumac), R. glabra (shining sumac), and Toxicodendron radicans (poison ivy). Cotinus is the classic name for wild olive and obovatus refers to the leaf shape (Vines 1960). There are only two species in the genus Cotinus; C. coggygria (European smoke tree) and C. obovatus (North American smoke tree). Cotinus coggygria is widely planted in the United States as an ornamental tree, but its native range extends from Europe east to the Himalayas (Elias). Cotinus obovatus occurs in seven states in the southeastern United States (Little 1943) and six counties in Oklahoma (Figure 4; Johnson and Hoagland 2004). Cotinus obovatus grows on calcareous bluffs and ravines where limestone predominates. Associated trees and shrubs often include Fraxinus quadrangulata (blue ash), Philadelphus pubescens (mock orange), Staphylea trifolia (bladdernut), Quercus muehlenbergii (chinkapin oak), and Ulmus rubra (red elm). Fort Gibson dam in Wagoner County and Chandler Park in Tulsa are excellent locations for viewing $C$. obovatus.

Cotinus obovatus was first discovered in Oklahoma in 1919 by Thomas Nuttall. He encountered this tree on limestone cliffs along the Grand River 30 miles north of its confluence with the Arkansas River. This plant was in fruit and greatly resembled the European species, which was referred to as Rhus cotinus in the early $19^{\text {th }}$ century. In this vein, he named the plant Rhus cotinoides. The name Cotinus obovatus was described by Constantine Rafinesque in 1840. The second discovery of $C$. obovatus in Oklahoma was made by Ernest Palmer on 14 April 1928, 109 years after Nuttall's visit, at a site near Page in LeFlore County (Little 1943).

The wood of $C$. obovatus has no economic value to the timber industry, due to its small size, but it is rot resistant and has been used for fence posts in some regions of the United States. During the Civil War, a yellow dye was extracted from the wood (Elias 1987). Currently C. coggygria is sold and planted as an ornamental plant in greater quantity than $C$. obovatus. However, its beautiful panicles of flowers in the spring and brilliant autumn colors make it a worthy addition to any home garden as well.

\section{LITERATURE CITED}

Elias, T.S. 1987. Trees of North America: a field guide and natural history. Gramercy Pub. Co., New York.

Hightshoe, G.L. 1988. Native trees, shrubs, and vines for urban and rural America: a planting design manual for environmental designers. Van Nostrand Reinhold, New York.

Johnson, F.L. and B.W. Hoagland. 2004. Catalog of the Woody Plants of Oklahoma [online]. Available: www.biosurvey.ou.edu. (Accessed on 1 June 2004).

Kurz, D. 1997. Shrubs and woody bines of Missouri. Missouri Department of Conservation, Jefferson City.

Little, E.L. 1943. American smoketree (Cotinus obovatus Raf.), one of Oklahoma's rarest tree species. Proceedings of the Oklahoma Academy of Science 23: 21-23.

Little, E.L., Jr., 1977, Atlas of United States trees, volume 4, minor Eastern hardwoods: U.S. Department of Agriculture Miscellaneous Publication 1342.

Little, E. L. 1996. Forest trees of Oklahoma, $14^{\text {th }}$. Oklahoma Department of Agriculture, Forestry Services.

Sargent, C.S. 1905. Manual of the trees of North America. Houghton Mifflin, New York.

Vines, R.A. 1960. Trees, shrubs, and woody vines of the southwest: a guide for the states of Arkansas, Louisiana, New Mexico, Oklahoma, and Texas. University of Texas Press, Austin.

Hoagland, B.W. 


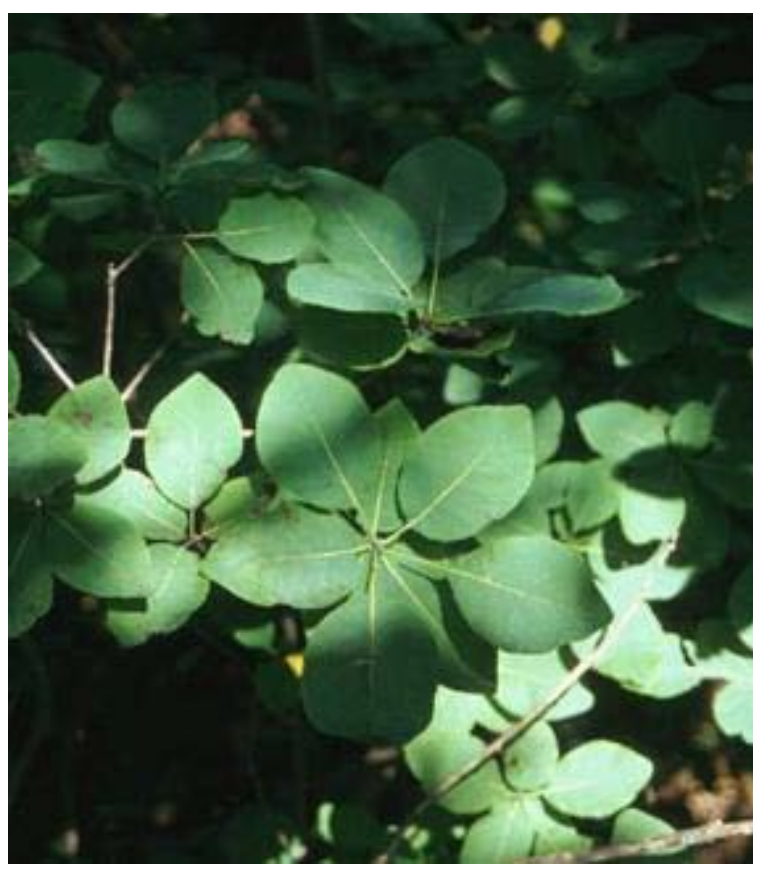

Figure 1 Leaves of Cotinus obovatus, Fort Gibson Dam, Wagoner County, Oklahoma.

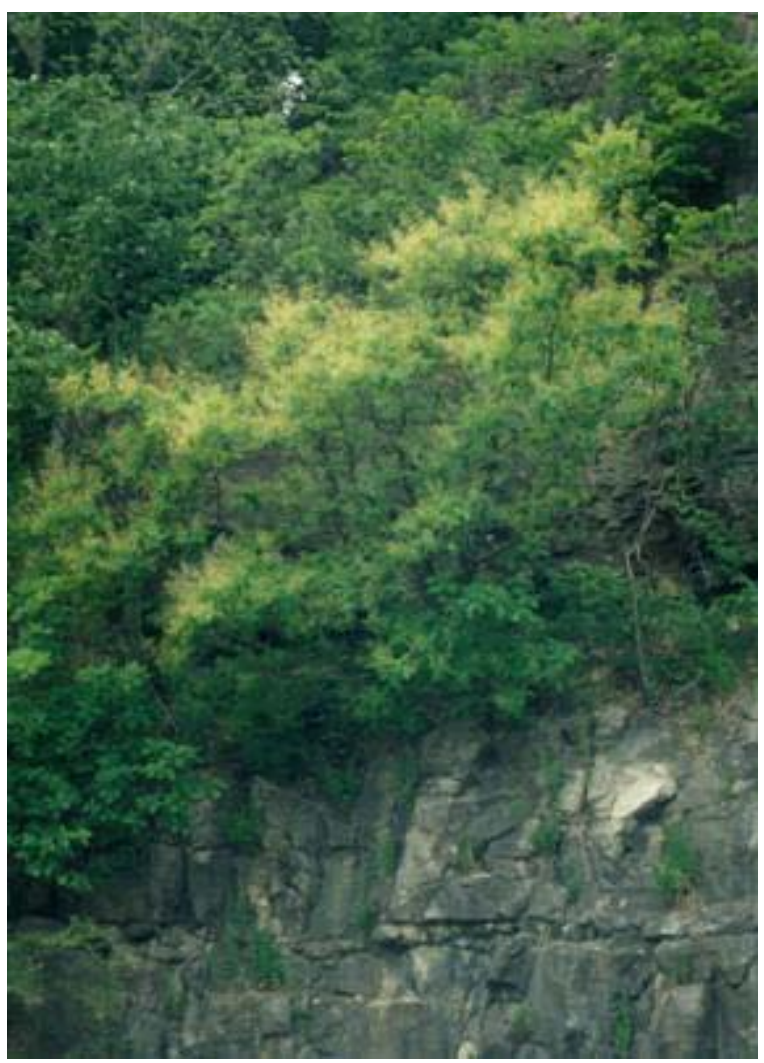

Figure 2 Habitat photo of Cotinus obovatus, Fort Gibson Dam, Wagoner County, Oklahoma.

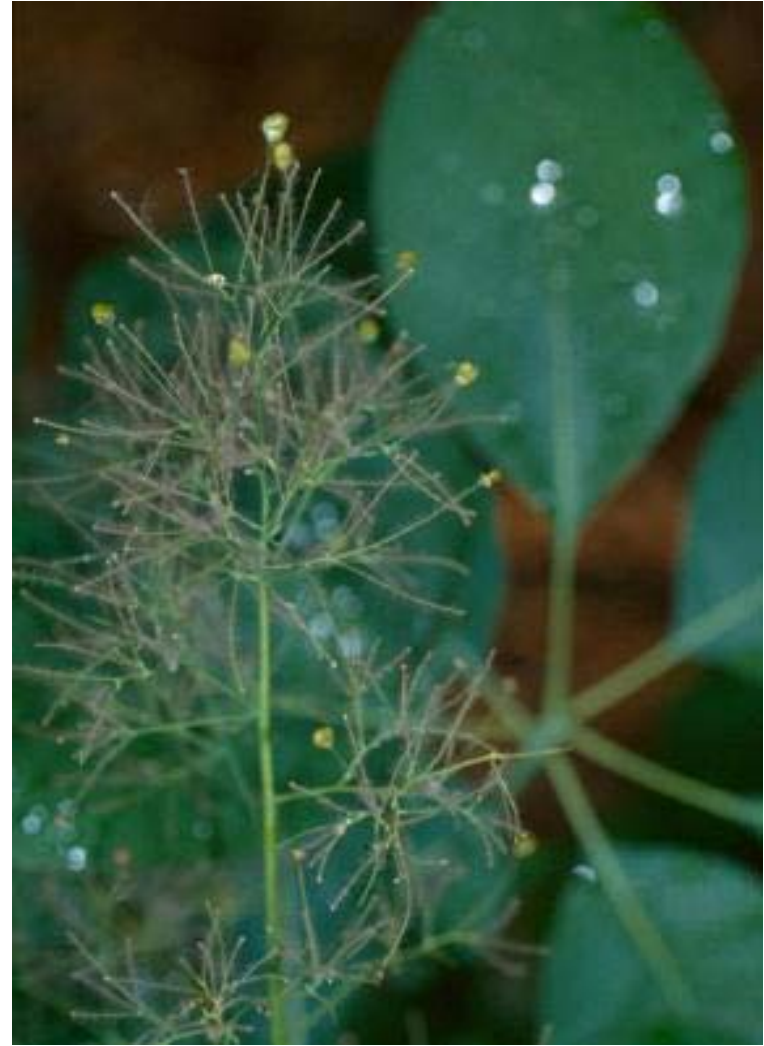

Figure 3 Inflorescence of Cotinus obovatus, Fort Gibson Dam, Wagoner County, Oklahoma.

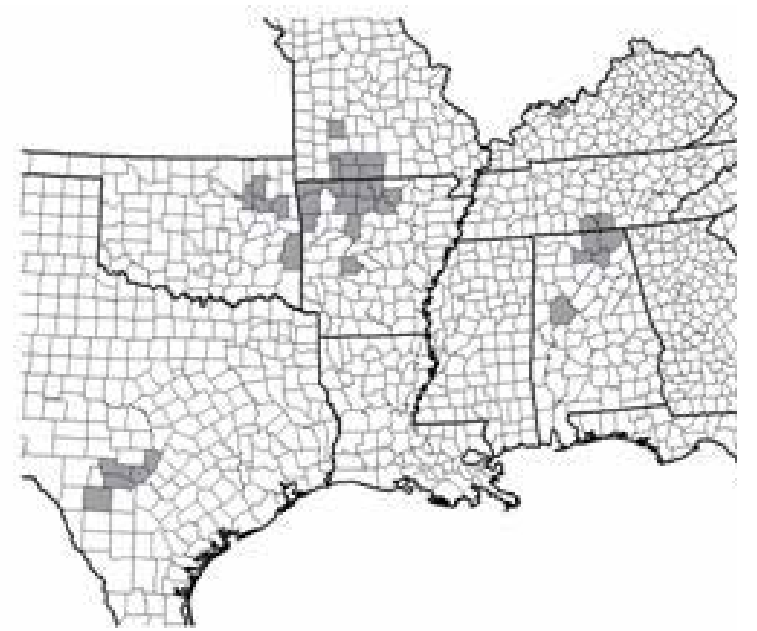

Figure 4 Distribution of Cotinus obovatus in North America (Adapted from Little 1977). 


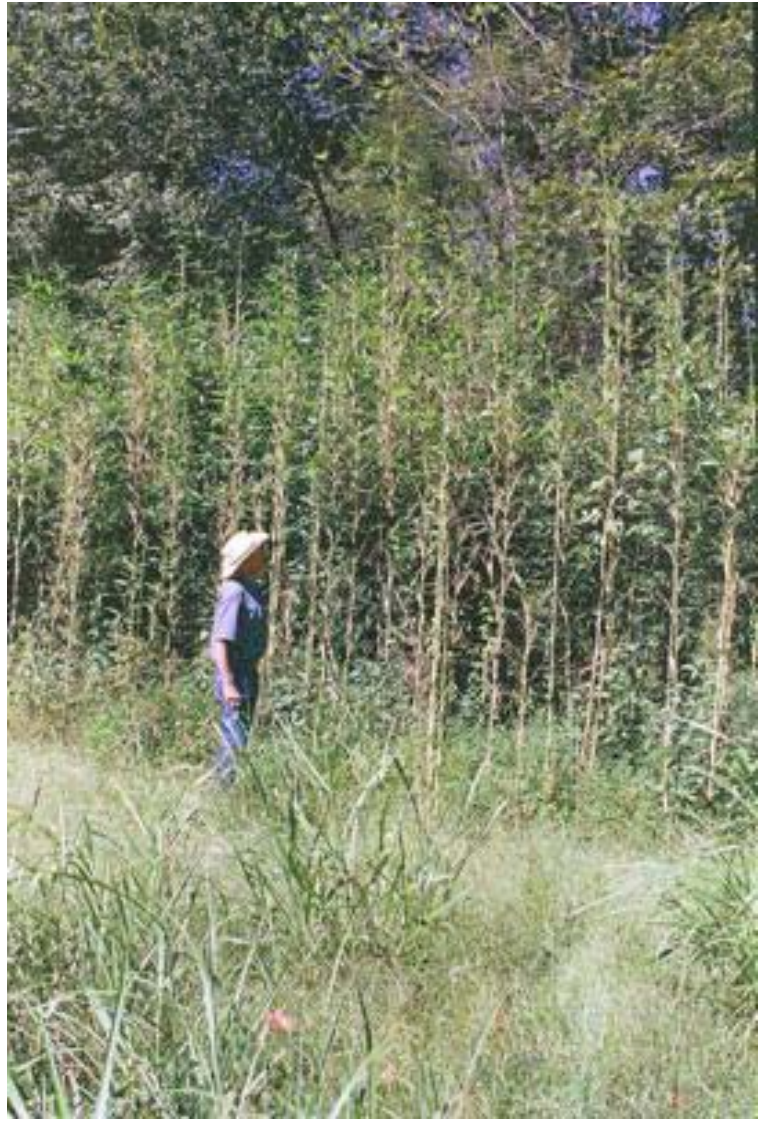

Figure 1 Stand of Giant Cane in Cherokee Countv. Oklahoma.

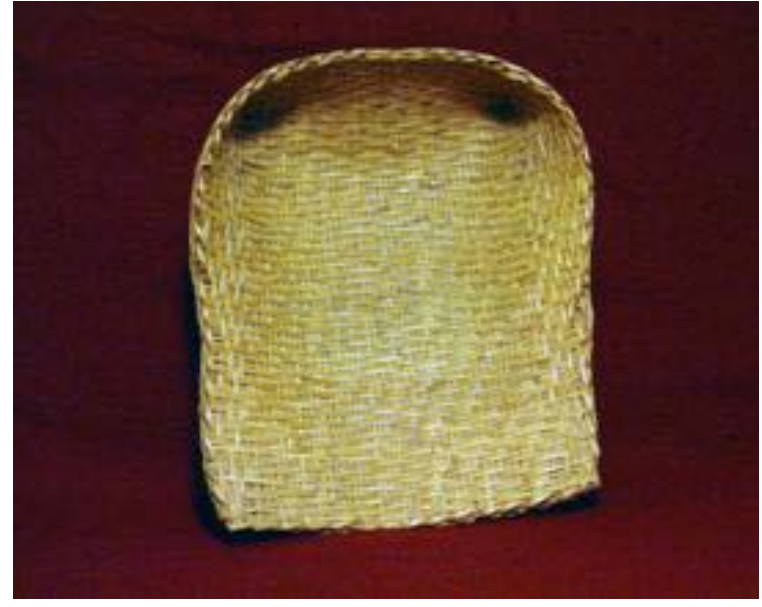

Figure 2 Fanner or winnowing basket $\left(\right.$ obfko $o^{\prime}$.

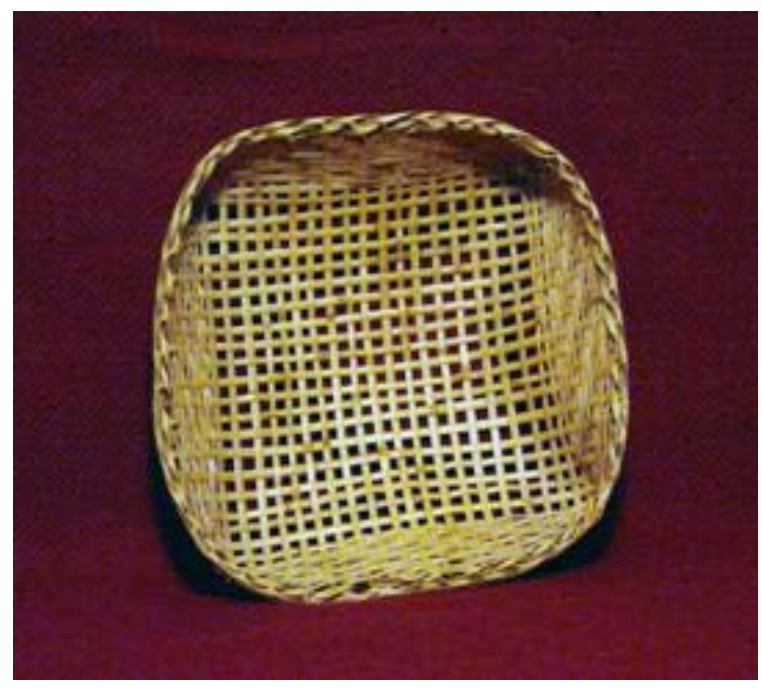

Figure 3 Sifter or sieve (ishsho'ha).

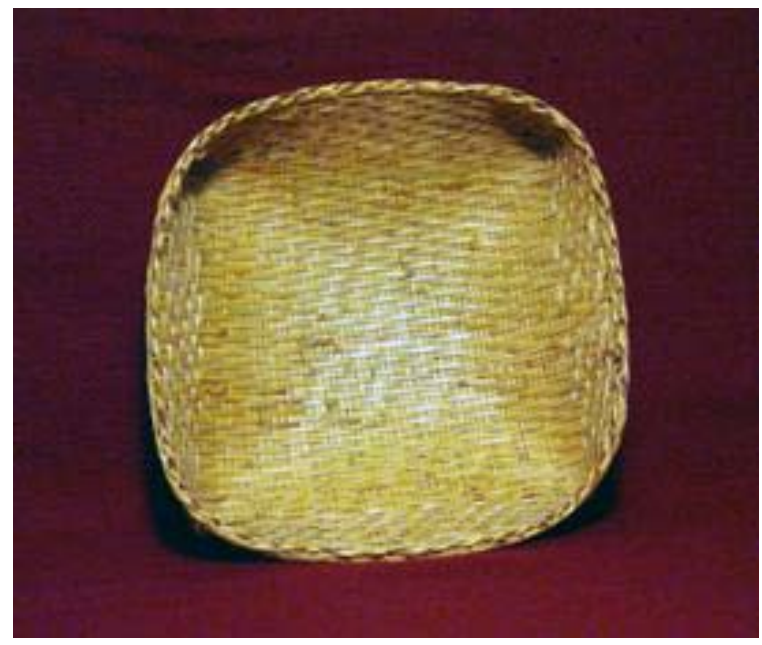

Figure 4 Utility basket, a shallow container. or tray (tapa).

Jordan, J.A.

https://doi.org/10.22488/okstate.17.100029 


\section{Giant Cane and Southeastern Indian Baskets}

\section{Ms. Julia A. Jordan \\ $23384^{\text {th }}$ Avenue, N.E. Norman, Oklahoma 73026}

Among the wide variety of natural materials suitable for basket making, one of the most attractive is giant cane (Figure 1), an Oklahoma native plant. Taxonomically, giant cane is in the genus Arundinaria, and the family Poaceae (grasses). This genus comprises the only native species of bamboo in the continental United States. Hitchcock (1971) recognized one genus: Arundinaria Michx. (cane) and two species: Arundinaria macrosperma Michx., giant cane, and Arundinaria tecta Walt. Muhl., switch cane. However Estes and Thompson (1984), following F. A. McClure, recognized one species, $A$. gigantea (Walter) Muhlenberg (cane) with three inclusive subspecies: A. gigantea ssp. gigantea, ssp. tecta (Walter) McClure, and ssp. macrosperma (Michaux.) McClure. Taylor and Taylor (1991) recognized one species, $A$. gigantea (Walt.) Muhl., giant cane. The taxon relevant to Oklahoma, and to southeastern Indian basketry generally, is $A$. gigantea ssp. gigantea, which will be referred to herein as giant cane.

Giant cane is a robust grass with culms (stems) reaching five meters or more in height and 5 to $8 \mathrm{~cm}(2-3$ in $)$ in diameter. It is the most widespread of the three subspecies, forming extensive colonies or canebrakes on the first and second terraces of major streams and wet lowlands. It is found in the Mississippi River Valley, the Appalachian-Ozarkian Uplands (including the Ouachita Highlands (USGS 2004), and the Gulf Coastal Plain (Estes and Thompson 1984), including much of eastern Oklahoma. It spreads rapidly by creeping horizontal rhizomes. The erect, woody culms are perennial--sometimes branching with flowering branchlets borne in fascicles on the main stem or on primary branches. Giant cane flowers infrequently and the flowering stems die after setting seed. Sterile branches, which are numerous, are branched repeatedly. The caryopses (seed grains) are large, up to $1.5 \mathrm{~cm}$ (0.5 in) long, floury, and are edible. They are produced in great abundance on each flowering stem. Swanton (1946) notes that they were used as food by southeastern Indians. Cane stalks grow rapidly, forming dense, tall stands that were formerly widespread and numerous in suitable habitats across the southeast. However, populations are now limited, probably due to the introduction of domestic animals and to the draining and clearing of fertile, lowland sites for agriculture. Both cattle and swine relish the young shoots, while pigs also root in the soil to consume the rhizomes.

Cane culms are jointed with hollow internodes. In contrast to most grasses, the stems are woody and there is extensive deposition of lignin and silica in the outer layer (Estes and Thompson 1984). This and the length of the fibers contribute to the strength of the stem. The culms are round in cross-section; thus they are lightweight and flexible, as well as strong. The hard, shiny surface of the culm results partly from a silica-wax cuticle which forms a thin layer over the silica-impregnated epidermis. The culm is therefore nearly impervious to water. These characteristics make giant cane an excellent material for the manufacture of many items of material culture, and it was utilized for many purposes by both aboriginal and historic Indian peoples of the southeastern United States. Swanton (1946) refers to cane as "one of the most important of all raw materials," for southeastern Indians. It was 
used for spears, arrows, blowguns, fishing crails and traps, beds, corncribs, flageolets, baskets, mats, and many other items. Blake and Cutler (2001) have recorded cane from prehistoric sites in Illinois, Indiana, and Arkansas, indicating some antiquity to the use of this material.

Giant cane was the favorite basketry material of such tribes as the Choctaw, Cherokee, Creek, Chitimacha, Natchez, and Caddo. Most southeastern basketry was made by the technique of weaving, as opposed to coiling (Hudson 1976). That is, weft (horizontal) elements were built up onto a warp (vertical) foundation. Twilling, in which two or more weft splints were passed over two or more warp splints, was the prevailing weave. The twilling technique produced a wide variety of diagonal and herringbone patterns, and when colored splints were combined with natural splints, the resulting baskets and mats were quite decorative, as well as useful.

Cane was usually converted into basketry splints immediately after gathering, though it could be processed later (Gettys 1984). The long lengths of cane were split lengthwise into quarters with a stout sharp knife. The object was to obtain a long and strong, flexible strip of even thickness. The splints were then trimmed along each edge to make them of uniform width, and scraped to a smooth texture on the inner surface. The glossy, natural, outer surface of the cane contributed to the beauty of cane baskets.

Some of the splints were dyed black, red, yellow, purple, or brown using dyes obtained from plants (Sinton 1946, Gettys 1984). A variety of mats and baskets were made. Large twilled cane mats, measuring about $152 \mathrm{~cm}$ by $183 \mathrm{~cm}$ ( $5 \mathrm{ft}$ by $6 \mathrm{ft}$ ), were used for bedding, for floor covering, to cover the seats in the square ground (summer council arena), to cover the walls and roofs of houses, and to wrap the bodies of the dead for burial. The finest
Southeastern baskets were double weave baskets, so called because they are woven with back-toback inside and outside fabrics, such that the surface of the basket was glossy and smooth both inside and out (Hudson 1976).

Like other tribes, the Choctaw produced many types of cane baskets for which they had names, including carrying baskets, hampers, pack baskets, trays, and pointed baskets. Of special importance was a three-piece set of baskets used in the preparation of hominy, a dietary staple. The set consisted of a winnowing basket or "fanner," (obfko') (Figure 2), a sieve or "sifter," (ishsho'ba) (Figure 3), and a shallow container or tray (tapa) (Figure 4), (Bushnell 1909). Collectively, this trilogy of baskets came to be called "Tom Fuller" baskets, the term deriving from the Choctaw word for hominy, tanfula (Edwards 1932).

Hominy was made from whole kernels of dried corn which were first soaked in cool water to which had been added some wood-ash lye (Hudson 1976). The next day the corn was drained and pounded in a mortar to loosen the hulls and crack the grains. The cracked corn was then separated from the hulls with the "fanner," a large flat basket with a shallow pocket at one end. The corn was placed in the basket which was then agitated up and down and back and forth to separate the heavier hominy from the lighter hulls. The "sifter" had a loosely woven plaited bottom through which the smaller grains could be separated from the coarser grains. The latter were returned to the mortar for further cracking. The tightly-woven cane tray had many uses, such as holding cracked and uncracked hominy, corn meal, and bread.

The accompanying photographs are of "Tom Fuller" baskets made of giant cane and purchased by the author in 1977 from a Choctaw basket maker of Wright City, Oklahoma. Fine quality cane baskets are

Jordan, J.A. 
produced today by the Mississippi Choctaw and the Chitimacha of Louisiana. Their sales outlets may be easily located on the internet. Gettys (1984) knew of only three cane weavers in Oklahoma (one of whom had produced this author's baskets), and believed that traditional forms not adaptable to modern uses had been dropped. Although it is highly unlikely that any "Tom Fuller" sets are now made for general sale, it is quite possible that a few Oklahoma Choctaw artisans are capable of filling a special order. Inquiry might begin with the Choctaw Nation Tribal Complex Office in Durant or at museums and specialty shops featuring authentic southeastern Indian arts and crafts.

\section{REFERENCES}

Blake, Leonard W. and Hugh C. Cutler. 2001. Plants from the past. Tuscaloosa: The University of Alabama Press.

Bushnell, David I., Jr. 1909. The Choctaw of Bayou Lacomb, St. Tammany Parish, Louisiana. U.S. Smithsonian Institution, Bureau of American Ethnology, Bulletin 48. Washington, D.C.: U.S. Government Printing Office.

Edwards, John. 1932. The Choctaw Indians in the middle of the nineteenth century. Chronicles of Oklahoma 10:3.
Estes, James R. and Rahmona A. Thompson. 1984. Cane: its characteristics and identification in baskets. In: Marshall Gettys (Ed.). Basketry of southeastern Indians. Idabel, OK: Museum of the Red River; p 57-64.

Gettys, Marshall. 1984. The Choctaw basket tradition. In: Marshall Gettys (Ed.). Basketry of southeastern Indians. Idabel, OK: Museum of the Red River, p 35-42.

Hitchcock, A. S. 1971. Manual of the grasses of the United States. Vol. 1, $2^{\text {nd }}$ ed., rev. Agnes Chase, New York: Dover Publications, Inc.

Hudson, Charles. 1976. The southeastern Indians. Knoxville: University of Tennessee Press.

Swanton, John R. 1946. The Indians of the southeastern United States. Vol. 1, $2^{\text {nd }}$ ed., rev. Agnes Chase, New York: Dover Publications, Inc.

Taylor, John R. and Constance E. S. Taylor. 1991. An annotated list of the ferns, fern allies, gymnosperms and flowering plants of Oklahoma. $2^{\text {nd }}$ ed. Durant, OK: [Published by the authors at Southeastern Oklahoma State University.]

USGS, 2004. Geology in the Parks. http://wrgis.wr.usgs.gov/docs/usgnpsnps Lprovince/inthigh.html 


\section{Vascular Flora of the Chouteau Wildlife Management Area Wagoner County, Oklahoma}

\author{
Bruce W. Hoagland \\ Oklahoma Biological Survey \\ and Department of Geography \\ University of Oklahoma \\ Norman, OK 73019 \\ e-mail: bhoagland@ou.edu
}

\author{
Forrest Johnson (deceased) \\ Oklahoma Biological Survey \\ University of Oklahoma \\ Norman, OK 73019
}

This article reports the results of a vascular plant inventory of the Chouteau Wildlife Management Area in eastern Oklahoma. One hundred eighty-one species of vascular plants were collected from 144 genera and 63 families. The families with the greatest number of species were the Asteraceae (25), Poaceae (22), and Fabaceae (18). Fifty-seven species were annuals, four biennials, and 120 were perennials. Thirty-nine woody plant species were present. Twenty-one species exotic to North America were collected representing $\mathbf{1 1 . 6 \%}$ of the flora. Azolla caroliniana was the only species tracked by the Oklahoma Natural Heritage Inventory found. This study reports 148 species previously not documented in Wagoner County.

\section{INTRODUCTION}

The objectives of this study were twofold: to fill a gap in floristic data for eastern Oklahoma and provide resource managers at the Chouteau Wildlife Management Area (CHWMA) with a comprehensive species list. Prior to 1996, when collecting began for this study, 198 specific and infraspecific taxa were reported from Wagoner County (Hoagland 2004). The first collections made in Wagoner County were by Robert Bebb, namesake of the University of Oklahoma Herbarium, in 1903 (Hoagland 2004). No additional collections were recorded until 1913, when G. W. Stevens visited the county. The peak collecting year in Wagoner County was 1939 (51 specimens), with work completed by R. Bebb (Hoagland et al. 2004).

\section{STUDY AREA}

The CHWMA is located on U.S. Army Corp of Engineers land in Wagoner County (Figure 1) and has been managed by the Oklahoma Department of Wildlife Conservation since 1973. It encompasses 402 hectares, and elevation ranges from $167 \mathrm{~m}$ to $158 \mathrm{~m}$. Latitudinal extent ranges

Hoagland, B.W. and Johnson, F.L.

https://doi.org/10.22488/okstate.17.100030 from $35.860 \mathrm{~N}$ to $35.85 \mathrm{o} \mathrm{N}$ and longitudinal extent from $95.34 \mathrm{o} \mathrm{W}$ to $95.37 \mathrm{o} \mathrm{W}$. The CHWMA is located within the subtropical humid (Cf) climate zone (Trewartha 1968). Summers are warm (mean July temperature $=27.7 \mathrm{o} C$ ) and humid, whereas winters are relatively short and mild (mean January temperature $=2.90 \mathrm{C}$ ). Mean annual precipitation is $114.5 \mathrm{~cm}$, with periodic severe droughts (Oklahoma Climatological Survey 2004).

Physiographically, the study area is located in the Osage Plains section of the Central Lowlands province (Hunt 1974) and within the Claremore Cuesta Plains province of Oklahoma (Curtis and Ham 1979). The surface geology is primarily Quaternary silt, sand, and clays deposited along the Verdigris River (Branson and Johnson 1979). The primary soil association at CHWMA is the Sage-Radley, which is composed of deep, level to gently sloping, poorly drained soils (Polone 1976). The potential natural vegetation type at CHWMA is the bottomland Forest type (Duck and Fletcher 1943). 


\section{METHODS}

Three collection sites were established at CHWMA for intensive floristic sampling. Sites were selected following a review of US Geological Survey 1:24,000 topographic maps and field reconnaissance. The predominant vegetation associations at these sites were classified according to Hoagland (2000). Collections also were made randomly throughout the site. Collections were made on a monthly basis from March through October 1996. Vouchers for species exotic to North America were made from naturalized populations only, thus excluding cultivated and ornamental plants. Specimens were processed at the Robert Bebb Herbarium of the University of Oklahoma (OKL) following standard herbarium techniques. Specimens were identified using Waterfall (1969) and Diggs et al. (1999). Origin (whether native or introduced to North America) was determined using Taylor and Taylor (1991) and United States Department of Agriculture-Natural Resources Conservation Service (USDA-NRCS 2004). Nomenclature follows USDA-NRCS (2004). Voucher specimens were deposited at OKL.

\section{RESULTS AND DISCUSSION}

A total of 181 vascular plants in 144 genera and 63 families were collected (Table 1). Among the angiosperms, 43 were monocots and 142 were dicots. The most species were collected from the families Poaceae (22), Asteraceae (25), Fabaceae (18). The genera Polygonum (6) and Carex (5) had the most species. Fifty-seven species were annual, four biennials, and 119 perennial. Thirty-nine woody plant species were present.

Twenty-one exotic species were collected, representing $11.6 \%$ of the flora. The numbers of exotic species were greater in the families Poaceae (6) and Fabaceae (7). These numbers are comparable to recent floristic inventories from other areas in Oklahoma. For example, a flora of the Chickasaw National Recreation Area reported 12\% exotic species (Hoagland and Johnson 2001),
9\% at Oologah Wildlife Management Area (Hoagland and Wallick 2003), 15\% at Keystone Wildlife Management Area (Hoagland and Buthod 2003), and 11\% for an inventory of Tillman County (Hoagland et al. 2004). However, the percentage was lower, 6.6\%, at Red Slough and Grassy Slough in southeastern Oklahoma (Hoagland and Johnson 2004). However, these studies report a higher number of exotic species in the Asteraceae. In addition, CHWMA is the first reported location for Alternanthera philoxeroides in Oklahoma, a noxious weed of the southeastern United States (Hoagland and McCarty 1998). Azolla caroliniana (G5S2) was the only species tracked by the Oklahoma Natural Heritage Inventory found at CHWMA. Species are ranked according to level of imperilment at the state $(S)$ and global $(G)$ levels on a scale of $1 \cdot 5 ; 1$ representing a species that is imperiled and 5 representing one that is secure (Groves et al. 1995).

As a result of this study, 313 species are now known to occur in Wagoner County. Of the 181 species reported in this study, 33 had been previously collected in the county. There were 165 species reported in the Atlas of the Flora of Oklahoma database that were not reported in this study (Hoagland 2004). This study documented 148 species not previously reported from Wagoner County. The three collection sites occurred within four vegetation associations. A brief description of each follows:

\section{Aquatic and wetland vegetation}

Several aquatic and wetland vegetation types were present at CHWMA. All intergraded with one another, making clear delineations difficult. The predominant emergent wetland vegetation types were Jussiaea peploides - Polygonum bydropiperoides herbaceous association, Nelumbo lutea herbaceous association, and Juncus effusus herbaceous association. Cephalantbus occidentalis shrubland association was the predominant woody wetland vegetation type. Associated 
species included Hibiscus laevis, Justicia americana, Potamogeton nodosus, Polygonum lapathifolium, P. pensylvanicum, Salix nigra, and Typha domingensis.

Azolla caroliniana, a species tracked by the Oklahoma Natural Heritage Inventory (2004), was found in this habitat type.

\section{Quercus palustris - Carya illinoensis/Ilex decidua forest association}

This association was the predominant forest type at CHWMA. However, all stands were immature second growth. Associate species included Amorpha fruticosa, Ampelopsis cordata, Arundinaria gigantea, Fraxinus pennsylvanica, Gleditsia triacanthos, Passiflora lutea and Ulmus rubra. On natural levies along the Verdigris River this association intergraded with the Acer saccharinum - Acer negundo forest association.

\section{Disturbed areas and old-field vegetation}

This designation included areas which have been or are currently in cultivation, roadsides and areas visited by CHWMA visitors, and other areas exhibiting signs of physical disruption. Common plants in disturbed areas and old fields included: Ambrosia trifida, Geranium carolinianum, Melilotus officinalis, Oenothera biennis, Solanum carolinense, Sorghum halepense, and Trifolium dubium.

\section{ACKNOWLEDGMENTS}

This project was funded by a grant from the Oklahoma Department of Wildlife Conservation.

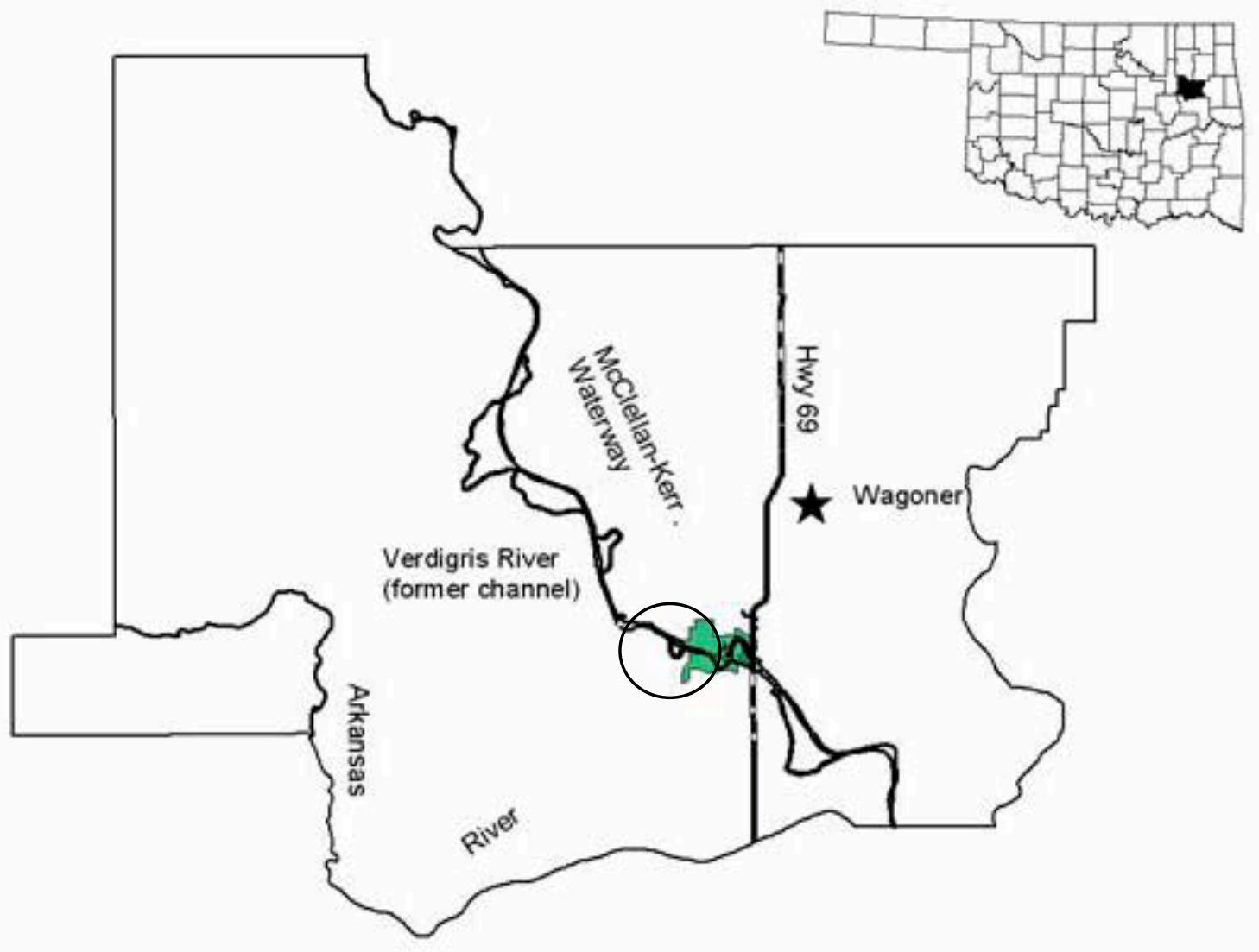

Figure Location of Chouteau Wildlife Management Area, Wagoner County, Oklahoma, site of the floristic collection.

Hoagland, B.W. and Johnson, F.L. 


\section{Annotated Species List for the Chouteau Wildlife Management Area}

The first entry is life history ( $\mathrm{A}=$ annual, $\mathrm{B}=$ biennial, $\mathrm{P}=$ perennial); followed by abundance $(1=$ least $5=$ dominant or codominant, Palmer et al. 1995); species not native to North America designated with an asterisk $\left(^{*}\right)$; habitat $(\mathrm{AQ}=$ Aquatic and wetland vegetation, $\mathrm{BF}=$ Quercus palustris $\bullet$ Carya illinoensis/Ilex decidua forest association, and DAOF=disturbed area/old-field); and collection number. Voucher specimens were deposited at the Robert Bebb Herbarium at the University of Oklahoma (OKL).

\section{PTERIDOPHYTA}

\section{Azollaceae}

Azolla caroliniana Willd. (mosquito fern) A; 2; AQ; CH096

MAGNOLIOPHYTA

MAGNOLIOPSIDA

\section{Acanthaceae}

Justicia americana (L.) Vahl (water willow) P; 2; AQ; CH037

Ruellia strepens $\mathrm{L}$. (wild petunia)

P; 2; BF; CH0173

Aceraceae

Acer negundo L. (boxelder)

P; 3; BF; CH079

A. saccharinum $\mathrm{L}$. (silver maple)

P; 2; BF; CH078

Amaranthaceae

Alternanthera philoxeroides (Mart.) Griseb.*

(alligator weed)

P; 3; AQ; CH094

Amaranthus palmeri $\mathrm{S}$. Wats.

(Palmer's pigweed)

A; 2; DAOF; CH0144

Apiaceae

Limnosciadium pinnatum (DC.) Mathias \&

Constance (tansy dog shade)

A; 3; AQ; CH065

Ptilimnium capillaceum (Michx.) Raf.

(threadleaf mockbishopweed)

A; 2; DAOF; CH0134

Sanicula canadensis L. (snakeroot)

B; 2; BF; CH0143

Torilis arvensis (Huds.) Link.*

(hedge parsley)

A; 2; DAOF; CH063

Apocynaceae

Apocynum cannabinum L. (Indian hemp)

P; 3; DAOF; CH085

Aquifoliaceae

Ilex decidua Walt. (deciduous holly)
P; 3; BF; CH0114

\section{Aristolochiaceae}

Aristolochia tomentosa Sims (wooly pipe vine)

P; 2; BF; CH0101

\section{Asclepiadaceae}

Asclepias incarnata L. (swamp milkweed)

P; 2; AQ; CH0160

A. viridis Walt. (green milkweed)

P; 2; DAOF; CH072

Asteraceae

Ageratina altissima (L.) King \& H.E. Robins.

(white snakeroot)

P; 2; DAOF; CH0194

Ambrosia artemisiifolia L. (common ragweed)

A; 3; DAOF; CH0174

A. trifida L. (giant ragweed)

A; 4; DAOF; CH0157

Bidens aristosa (Michx.) Britt.

(bearded beggarticks)

A; 2; AQ; CH0206

Boltonia asteroides (L.) L'Her. var. latisquamata

(Gray) Cronq. (white doll's daisy)

P; 2; AQ; CH0208

Cirsium altissimum (L.) Hill (tall thistle)

B; 2; DAOF; CH0185

Conoclinium coelestinum (L.) DC.

(blue mistflower)

P; 2; AQ; CH0199

Conyza canadensis (L.) Cronq. (horseweed)

A; 3; DAOF; CH0162

Coreopsis tinctoria Nutt. (plains coreopsis)

A; 3; DAOF; CH0123

Dracopis amplexicaulis (Vahl.) Cass.

(clasping coneflower)

A; 4; AQ, DAOF; CH073

Eclipta prostrata (L.) L. (yerba de tajo)

P; 3; AQ; CH0108

Elephantopus carolinianus Raeusch.

(elephant's foot)

P; 2; BF; CH0150 
Erigeron strigosus Muhl. ex Willd.

(daisy fleabane)

B; 2; DAOF; CH090

Grindelia papposa Nesom \& Suh (goldenweed)

A; 2; DAOF; CH0111

Helianthus annuus L. (common sunflower)

A; 2; DAOF; CH0164

Iva annua $\mathrm{L}$. (marsh elder)

A; 3; DAOF; CH0158

Lactuca serriola L.* (prickly lettuce)

A; 2; DAOF; CH0145

Pyrrhopappus multicaulis (D. Don) DC.

(Geiser's false dandelion)

P; 2; DAOF; CH060

Solidago canadensis L. (Canada goldenrod)

P; 2; DAOF; CH0197

Symphyotrichum ericoides (L.) Nesom

(white heath aster)

P; 2; DAOF; CH0189

S. ontarione (Wieg.) Nesom (bottomland aster)

P; 2; DAOF; CH0200

S. subulatum (Michx.) Nesom (eastern

saltmarsh aster)

A; 4; AQ; CH0165

Verbesina virginica $\mathrm{L}$. (frostweed)

P; 2; BF; CH0184

Vernonia baldwinii Torr. (western ironweed)

P; 2; DAOF; CH0163

Xanthium strumarium L. (cocklebur)

A; 2; AQ; CH0209

\section{Balsaminaceae}

Impatiens capensis Meerb. (jewelweed)

A; 2; BF; CH0109

Bignoniaceae

Campsis radicans (L.) Seem. ex Bureau

(trumpetvine)

P; 2; BF; CH083

Brassicaceae

Lepidium densiflorum Schrad. (peppergrass)

A; 2; DAOF; CH051

Rorippa palustris (L.) Bess (bog yellow cress)

A; 2; AQ; CH088

Thlaspi arvense L.* (field pennycress)

A; 1; DAOF; CH053

Campanulaceae

Triodanis perfoliata (L.) Nieuw.

(clasping Venus' looking glass)

A; 2; DAOF; CH082

\section{Caprifoliaceae}

Sambucus nigra L. ssp. canadensis (L.) R. Bolli (elderberry) P; 2; BF; CH084

Viburnum rufidulum Raf. (rusty blackhaw)

P; 2; BF; CH074

Celastraceae

Euonymus atropurpurea Jacq. (wahoo)

P; 2; BF; CH0187

Chenopodiaceae

Chenopodium standleyanum Aellen

(Standley's goosefoot)

A; 3; DAOF; CH0159

\section{Convolvulaceae}

Ipomoea lacunosa L. (white morning glory)

A; 2; DAOF; CH0203

I. pandurata (L.) G.F.W. Mey.

(bigroot morning glory)

P; 3; DAOF; CH0129

\section{Cornaceae}

Cornus drummondii C.A. Mey.

(rough leaved dogwood)

P; 3; DAOF; CH069

Crassulaceae

Penthorum sedoides L. (ditch stonecrop)

P; 3; AQ; CH0176

Ebenaceae

Diospyros virginiana $\mathrm{L}$. (persimmon)

P; 2; DAOF; CH025

Euphorbiaceae

Chamaesyce maculata (L.) Small

(spotted spurge)

A; 3; DAOF; CH0151

Euphorbia spathulata Lam. (warty spurge)

A; 2; DAOF; CH049

Fabaceae

Amorpha fruticosa L. (false indigo)

P; 2; AQ; CH052

Cercis canadensis $\mathrm{L}$. (redbud)

P; 3; BF; CH0170

Desmanthus illinoensis (Michx.) MacM. ex B.L.

Robins. \& Fern. (bundleflower)

P; 2; DAOF; CH0125

Desmodium paniculatum (L.) DC.

(panicled tickclover)

P; 3; BF; CH0106

Gleditsia triacanthos L. (honey locust)

P; 3; BF; CH044

Gymnocladus dioicus (L.) K. Koch.

Hoagland, B.W. and Johnson, F.L. 
(Kentucky coffee tree)

P; 2; BF; CH092

Lathyrus pusillus Ell. (low peavine)

A; 2; DAOF; CH0O2

Lespedera cuneata (Dum.-Cours.) G. Don*

(sericea lespedeza)

P; 2; DAOF; CH0167

Melilotus alba Medikus* (white sweet clover) A; 2; DAOF; $\mathrm{CH} 071$

M. officinalis (L.) Lam.* (yellow sweet clover) A; 3; DAOF; CH041

Senna marilandica (L.) Link (wild senna)

P; 2; BF; CH0124

Sesbania herbacea (P. Mill.) McVaugh (bequilla)

A; 5; AQ; CH0166

Strophostyles helvola (L.) Ell.

(fuzzy trailing bean)

A; 2; DAOF; CH0191

Trifolium arvense L.* (rabbit foot clover)

A; 2; DAOF; $\mathrm{CH} 040$

T. dubium Sibthrop* (small hop clover)

A; 2; DAOF; $\mathrm{CH} 026$

T. pratense L.* (red clover)

P; 2; DAOF; CH0140

Vicia caroliniana Walt. (pole vetch)

P; 3; DAOF; CH0128

$V$. villosa Roth* (hairy vetch)

A; 3; DAOF; CH035

Fagaceae

Quercus macrocarpa Michx. (bur oak)

P; 2; BF; CH0135

Q. palustris Muenchh. (pin oak)

P; 3; BF; CH034

Q. velutina Lam. (black oak)

P; 2; BF; CH042

Geraniaceae

Geranium carolinianum L. (Carolina cranesbill)

A; 2; DAOF; $\mathrm{CH} 027$

Juglandaceae

Carya illinoensis (Wangenh.) K. Koch (Pecan)

P; 2; BF; CH087

Lamiaceae

Prunella vulgaris L. (Common self heal)

P; 2; BF; CH020

Lauraceae

Sassafras albidum (Nutt.) Nees (sassafras)

P; 2; BF; CH0120

\section{Lythraceae}

Ammannia coccinea Rottb. (redstem loosestrife)

A; 2; AQ; CH0141

Lythrum alatum Pursh (winged loosestrife)

P; 2; AQ; CH0121

Malvaceae

Hibiscus laevis All. (halberd leaved rose

mallow) P; 2; AQ; CH0153

Sida spinosa L. (prickly sida)

A; 1; DAOF; CH0152

Menispermaceae

Calycocarpum lyonii (Pursh) Gray (cupseed)

$\mathrm{P} ; 2$; BF; CH093

Cocculus carolinus (L.) DC. (Carolina snailseed)

P; 2; BF; CH0103

Moraceae

Morus rubra L. (red mulberry)

P; 2; BF; CH0180

Nelumbonaceae

Nelumbo lutea Willd. (Lotus)

P; 2; AQ; CH0179

Oleaceae

Fraxinus pennsylvanica Marsh. (green ash)

P; 3; BF; CH043

\section{Onagraceae}

Ludwigia palustris (L.) Ell. (marsh seedbox)

P; 4; AQ; CH055

L. repens Forst. (water primrose)

P; 2; AQ; CH0131

Oenothera biennis L.

(common evening primrose)

B; 3; DAOF; CH0161

O. laciniata Hill (cutleaf evening primrose)

A; 2; DAOF; CH061

Oxalidaceae

Oxalis stricta L. (yellow wood sorrel)

P; 2; DAOF; CH081

Passifloraceae

Passiflora lutea L. (yellow passionflower)

P; 2; BF; CH058

Phytolaccaceae

Phytolacca americana L. (pokeweed)

P; 2; DAOF; CH0116

Polygonaceae

Polygonum hydropiper L.* (water pepper)

A; 2; AQ; CH0115

P. bydropiperoides Michx.* (mild water pepper)

P; 4; AQ; CH0113 
P. lapathifolium L. (pale smartweed)

A; 3; AQ; CH0190

P. pensylvanicum L. (Pennsylvania smartweed)

A; 2; AQ; CH0204

P. ramosissimum Michx. (knotweed)

A; 2; AQ; $\mathrm{CH} 014$

P. scandens $\mathrm{L}$. (false buckwheat)

P; 2; AQ; CH0193

Rumex altissimus Wood (pale dock)

P; 2; DAOF; CH089

R. crispus L.* (curly dock)

P; 3; DAOF; CH091

R. verticillatus $\mathrm{L}$. (Water dock)

P; 2; DAOF; CH07

Ranunculaceae

Clematis pitcheri Torr. \& Gray (Pitcher's

clematis) P; 2; BF; CH046

Ranunculus sceleratus L. (cursed buttercup)

A; 2; AQ; CH031

\section{Rosaceae}

Crataegus viridis L. (green hawthorn)

P; 3; BF; CHO6

Geum canadense Jacq. (white avens)

P; 2; BF; CH0112

Rosa multiflora Thunb. ex Murr.*

(Japanese rose)

P; 2; DAOF; CH033

R. setigera Michx. (climbing prairie rose)

P; 2; DAOF; CH056

Rubus trivialis Michx. (southern blackberry)

P; 3; BF; CH0105

Rubiaceae

Cephalanthus occidentalis L. (buttonbush)

P; 2; AQ; CH0138

Galium aparine L. (catchweed bedstraw)

A; 2; BF; CH036

Spermacoce glabra Michx. (smooth buttonweed)

P; 2; AQ; CH0155

Salicaceae

Salix nigra Marsh. (black willow)

P; 2; AQ; CH0192

Sapindaceae

Sapindus saponaria L. var. drummondii

(Hook. \& Arn.) L. Benson (soapberry)

P; 2; BF; CH077

\section{Sapotaceae}

Sideroxylon lanuginosum Michx. (chittamwood) P; 2; BF; CH0110

\section{Scrophulariaceae}

Lindernia dubia (L.) Pennell (false pimpernel)

A; 2; AQ; CH0136

Penstemon digitalis Nutt. ex Sims

(smooth penstemon)

P; 2; DAOF; CH045

Veronica peregrina L. (purslane speedwell)

A; 2; DAOF; CH024

Solanaceae

Physalis angulata L. (cutleaf ground cherry)

A; 2; DAOF; CH015

Solanum carolinense $\mathrm{L}$. (Carolina horsenettle)

P; 2; DAOF; CH062

Ulmaceae

Celtis laevigata Willd. (sugarberry)

P; 4; BF; CH01

Ulmus alata Michx. (winged elm)

P; 3; BF; CH032

U. rubra Muhl. (slippery elm)

P; 4; BF; CH038

Urticaceae

Boebmeria cylindrica (L.) Sw. (false nettle)

P; 2; BF; CH0175

Valerianaceae

Valerianella radiata (L.) Dufr.

(common beaked cornsalad)

A; 2; AQ; CH08

Verbenaceae

Phyla lanceolata (Michx.) Greene

(northern fogfruit)

P; 2; AQ; CH0139

Viscaceae

Phoradendron leucarpum (Raf.) Reveal \& M.C.

Johnston (eastern mistletoe)

P; 2; BF; CH086

Vitaceae

Ampelopsis arborea (L.) Koehne (peppervine)

P; 2; BF; CH0100

A. cordata Michx. (racoon grape)

P; 2; BF; CH0147

Parthenocissus quinquefolia (L.) Planch.

(Virginia creeper)

P; 3; BF; CH098

Vitis aestivalis Michx. (pigeon grape)

P; 3; BF; CH0102

$V$. cinerea (Engelm.) Millard (sweet grape)

P; 2; BF; CH0107

Hoagland, B.W. and Johnson, F.L. 


\section{LILIOPSIDA}

\section{Alismataceae}

Echinodorus cordifolius (L.) Griesb.

(creeping burhead)

P; 2; AQ; CH0177

Sagittaria latifolia Willd. (duck potato)

P; 2; AQ; CH0186

\section{Araceae}

Arisaema dracontium (L.) Schott (green dragon) P; 2; BF; CH0114

\section{Cyperaceae}

Carex crus-corvi Shuttlw. ex Kunze

(ravenfoot sedge)

P; 2; AQ; CH070

C. granularis Muhl. ex Willd. var. haleana

(Olney) Porter (Limestone meadow

sedge)

P; 2 BF; CH0032

C. byalinolepis Steudel (shoreline sedge)

P; 2; AQ; CH0089

C. tribuloides Wahlenberg (blunt broom sedge)

P; 2 BF; CH0103

C. vulpinoidea Michx. (fox sedge)

P; 2 BF; CH0230

Cyperus pseudovegetus Stued.

(marsh flatsedge)

P; 2; AQ; CH0114

C. strigosus $\mathrm{L}$. (strawcolored flatsedge)

P; 2; AQ; CH097

Eleocharis compressa Sullivant

(flatstem spikesedge)

P; 4; AQ; CH052

E. obtusa (Willd.) J.A. Schultes

(blunt spikesedge)

P; 2; AQ; CH0039

\section{Iridaceae}

Sisyrinchium angustifolium P. Mill.

(blue eyed grass)

P; 2; DAOF; CH0029

Juncaceae

Juncus acuminatus Michx. (tapertip rush)

P; 2; AQ; CH063

J. effusus L. (soft rush)

P; 2; AQ; CH024

J. interior Wieg. (inland rush)

P; 2; AQ; CH041

Liliaceae

Allium canadense L. (wild onion)

$$
\text { P; 2; DAOF; CH030 }
$$

Poaceae

Agrostis byemalis (Walt.) B. S. P. (ticklegrass)

P; 2; AQ; CH0017

Alopecurus carolinianus Walt. (Carolina foxtail)

A; 2; AQ; CH0019

Andropogon glomeratus (Walt.) B. S. P.

(broomsedge)

P; 3; DAOF; CH0182

Arundinaria gigantea (Walt.) Mulh. (giant cane)

P; 2; BF; CH076

Bromus japonicus Thunb. ex Murr*.

(Japanese brome)

P; 3; DAOF; CH047

Digitaria sanguinalis (L.) Scop. (hairy crabgrass)

A; 3; DAOF; CH0169

Echinochloa colona (L.) Link* (barnyard grass)

A; 2; AQ; CH0205

E. crus-galli (L.) Beauv.* (barnyard grass)

A; 3; AQ; CH0104

E. muricata (Beauv.) Fern.* (barnyard grass)

A; 2; AQ; CH0130

Elymus virginicus $\mathrm{L}$. (Virginia wild rye)

P; 2; BF; CH075

Eragrostis spectabilis (Pursh.) Steud.

(purple lovegrass)

P; 2; BF; CH0196

Hordeum pusillum Nutt. (little barley)

A; 3; DAOF; CH050

Leersia oryzoides (L.) Sw. (rice cutgrass)

P; 2; AQ; CH0181

Leptochloa panicea (Retz.) Ohwi ssp. brachiata

(Steudl.) N. Snow (red sprangletop)

A; 2; AQ; CH0201

Lolium perenne L.* (perennial ryegrass)

P; 2; DAOF; CH048

Panicum dichotomiflorum Michx. (fall panicum)

A; 2; BF; CH0198

Paspalum pubiflorum Rupr. ex Fourn.

(hairyseed paspalum)

P; 2; DAOF; CH0202

Setaria parviflora (Poir.) Kerguélen.

(knotroot bristlegrass)

P; 2; DAOF; CH0207

S. viridis (L.) Beauv.* (green foxtail)

A; 2; DAOF; CH0127

Sorghum halepense (L.) Pers.*

(Johnson grass) 
P; 3; DAOF; CH021

Sphenopholis obtusata (Michx) Scribn.

(wedgegrass)

P; 2; AQ; CH010

Tridens flavus (L.) A.S. Hitchc. (redtop)

P; 3; DAOF; CH0183

Potamogetonaceae

Potamogeton nodosus Poir.

(long leaved pondweed)
P; 2; AQ; CH095

Smilacaceae

Smilax bona-nox L. (greenbriar)

P; 2; BF; CH097

$S$. glauca Walt. (pale greenbriar)

P; 2; BF; CH0119

Typhaceae

Typha domingensis Pers. (southern cattail)

P; 2; AQ; CH0178

Table Summary of floristic collections at the Chouteau Wildlife Management Area, Wagoner County, Oklahoma. Table format follows Palmer et al. (1995).

\begin{tabular}{llll}
\hline Taxonomic Group & Species & Native spp. & Introduced spp. \\
Pteridophyta & 1 & 1 & 0 \\
Magnoliophyta & 137 & 122 & 15 \\
Magnoliopsida & 43 & 37 & 6 \\
Liliopsida & 181 & 160 & 21 \\
\hline Total & & & \\
\hline
\end{tabular}

\section{LITERATURE CITED}

Branson, C.C. and K.S. Johnson. 1979.

Generalized geologic map of Oklahoma.

Page 4 in K.S. Johnson, C.C.

Branson, N.M. Curtis, W. E. Ham,

W.E. Harrison, M.V. Marcher, and

J.F. Roberts, editors, Geology and

Earth Resources of Oklahoma.

Oklahoma Geological Survey,

Norman.

Curtis, N.M. and W.E. Ham. 1979.

Geomorphic provinces of Oklahoma. Page
45 in K.S. Johnson, C.C. Branson, N.M. Curtis, W.E. Ham, W.E. Harrison, M.V. Marcher, and J.F. Roberts, editors, Geology and Earth Resources of Oklahoma. Oklahoma Geological Survey, Norman.

Diggs, G.M., B.L. Lipscomb, and R.J. O'Kennon. 1999. Shinners and Mabler's Illustrated Flora of North Central Texas. Botanical Research Institute of Texas and Austin College, Fort Worth.

Hoagland, B.W. and Johnson, F.L. 
Duck, L.G. and J.B. Fletcher. 1943. A game type map of Oklahoma. Oklahoma Department of Wildlife Conservation, Oklahoma City. Groves, C.R., M.L. Klein, and T.F. Breden. 1995. Natural Heritage Programs: public-private partnerships for biodiversity conservation. Wildlife Society Bulletin 23:784-790.

Hoagland, B.W. 2000. The vegetation of Oklahoma: a classification of landscape mapping and conservation planning. Southwest Naturalist 45:385-420.

Hoagland, B.W. 2004. Atlas of the flora of Oklahoma [online]. Available: www.biosurvey.ou.edu. (Accessed on 14 January 2004).

Hoagland, B.W. and A. Buthod. 2003. $V$ ascular flora of the Keystone Wildlife Management Area, Creek, Pawnee, and Osage counties, Oklahoma. Oklahoma Native Plant Record 3:23-37.

Hoagland, B.W., P. Crawford-Callahan, P. Crawford, and F.L. Johnson. 2004. V ascular Flora of Hackberry Flat, Frederick Lake, and Suttle Creek, Tillman County, Oklahoma. Sida 21:429-445.

Hoagland, B.W. and F.L. Johnson. 2001. Vascular flora of the Chickasaw National Recreation Area, Murray County, Oklahoma. Castanea 66:383-400.

Hoagland, B.W. and F.L. Johnson. 2004. The vascular flora of Red Slough and Grassy Slough Wildlife Management Areas, Gulf Coastal Plain, McCurtain County, Oklahoma. Castanea 69.

Hoagland, B. W. and N. A. McCarty. 1998. Alternanthera philoxeroides (Mart.) Griseb.(AMARANTHACEAE) new to Oklahoma. Castanea 63: 194.
Hoagland, B.W. and K. Wallick. 2003. Vascular flora of Oologah Wildlife Management Area, Nowata County, Oklahoma. Proceedings of the Oklahoma Academy 83:47-62.

Hunt, C.B. 1974. Natural Regions of the United States and Canada. W. H. Freeman, San Francisco.

Oklahoma Climatological Survey. 2004. Oklahoma Climatological Data [online]. Available: http://www.ocs.ou.edu/. (Accessed on 1 March 2004).

Oklahoma Natural Heritage Inventory. 2004. ONHI working list of rare Oklahoma plants [online]. Available: http://www.biosurvey. ou.edu/publicat.html. (Accessed on 1 March 2004).

Palmer, M.W., G.L. Wade, and P. Neal. 1995. Standards for the writing of floras. Bioscience 45:339-345.

Polone, D. J. 1976. Soil survey of $W$ agoner County, Oklahoma. United States Department of Agriculture, Washington D.C.

Taylor, R.J. and C.S. Taylor. 1991. An annotated list of the ferns, fern allies, gymnosperms, and flowering plants of Oklahoma. Southeastern Oklahoma State University, Durant.

Trewartha, G.T. 1968. An Introduction to Climate. McGraw-Hill, New York.

USDA-NRCS 2004. The PLANTS database [online].

Available: http://plants.usda.gov/pl ants. National Plant Data Center, Baton Rouge, LA. (Accessed on 14 January 2004).

Waterfall, U.T. 1969. Keys to the flora of Oklahoma. $4^{\text {th }}$ edition. Published by the author, Stillwater. 


\section{Status and Habitat Characteristics of Cypripedium kentuckiense (Kentucky lady's slipper) in Southeastern Oklahoma}

\author{
Amy K. Buthod \\ Oklahoma Biological Survey \\ University of Oklahoma \\ Norman, OK 73019 \\ e-mail: amybuthod@ou.edu
}

\author{
B. W. Hoagland \\ Oklahoma Biological Survey and \\ Department of Geography \\ University of Oklahoma \\ Norman, OK 73019 \\ e-mail: bhoagland@ou.edu
}

Cypripedium kentuckiense is a long-lived herbaceous perennial that inhabits floodplain and mesic hardwood forests. It occurs in Alabama, Kentucky, Louisiana, Mississippi, Oklahoma, Tennessee, Texas, and Virginia and has been reported from Choctaw, LeFlore, McCurtain, and Pushmataha counties in Oklahoma. $C$. kentuckiense is considered a rare species throughout its range, but is not currently protected under the United States Endangered Species Act. The objectives of this study were to (1) determine whether known populations of $C$. kentuckiense were persisting in Oklahoma and (2) characterize habitat structure. Twelve sites were surveyed in 2001 and 2002 for populations of $C$. kentuckiense, but only three persistent populations were found. The populations that were relocated numbered fewer than 20 total stems and all showed a dramatic decline in population size relative to previous surveys.

\section{INTRODUCTION}

Cypripedium kentuckiense is a long-lived herbaceous perennial that inhabits floodplain or mesic hardwood forests or woodland springs and seeps (Case et al., 1998; Reed 1982, Hooks 2000, Magrath 2001). Populations of C. kentuckiense occur in Alabama, Kentucky, Louisiana, Mississippi, Oklahoma, Tennessee, Texas, and Virginia (Figure; USDA 2002). In Oklahoma, C. kentuckiense has been reported from

Choctaw, LeFlore, McCurtain, and Pushmataha counties (Hoagland et al. 2004). Over 156 populations are known to exist throughout its range, the majority of which occur in Arkansas. Oklahoma harbors only $4.5 \%$ of C. kentuckiense populations (Atwood 1984, 1985; Case et al. 1998). Population size averages less than 20 individuals (Weldy et al. 1996), though some in Arkansas exceed 800 individuals (Hooks 2000).

Cypripedium. kentuckiense is considered a rare species throughout its range, but is not currently protected under the U.S. Endangered Species Act. Prior to 1996, it was listed as a category 2 (C2) species by the
U.S. Fish and Wildlife Service. A C2 species is defined as "... a likely candidate for federal listing as endangered or threatened, but it is necessary to obtain further information regarding possible threats" (Department of the Interior 1993).

State and federal agencies evaluate the conservation status of a species using a two tiered, geographical approach developed by The Nature Conservancy (Groves et al. 1995). This system ranks species imperilment at the state $(\mathrm{S})$ and $\operatorname{global}(\mathrm{G})$ levels on a scale of 1-5; 1 representing a species that is imperiled and 5 , one that is demonstrably secure. NatureServe, a conservation information organization, has assigned C. kentuckiense a global rank of G3, indicating a species that is "... either very rare and local throughout its range or found locally (even abundantly at some of its locations) in a restricted range, or because of other factors making it vulnerable to extinction throughout its range..." (NatureServe 2004). The Oklahoma Natural Heritage Inventory (ONHI) has assigned $C$. kentuckiense a state rank of S1, indicating a 
species “...critically imperiled... because of extreme rarity (five or fewer occurrences or very few remaining individuals or acres) or because of some factor of its biology making it especially vulnerable to extinction" (ONHI 2001). In comparison, Arkansas and Kentucky rank C. kentuckiense as S3 (Arkansas Natural Heritage Commission 2001; Kentucky Nature Preserves Commissions 2001), Tennessee S1S2

(Tenessee Department of Environment and Conservation 2001, and Alabama Louisiana, Mississippi, and Virginia as S1 (Alabama
Natural Heritage Program 1996; Louisiana Natural Heritage Program 2002; Mississippi Museum of Natural History 2002; Virginia Natural Heritage Program 2002) by Heritage Programs in those states. An S-rank was not available for Texas (Texas Conservation Data Center 2001.

The objectives of this study were (1) to verify and determine whether known populations of $C$. kentuckiense persist in Oklahoma and (2) to gather quantitative habitat data.
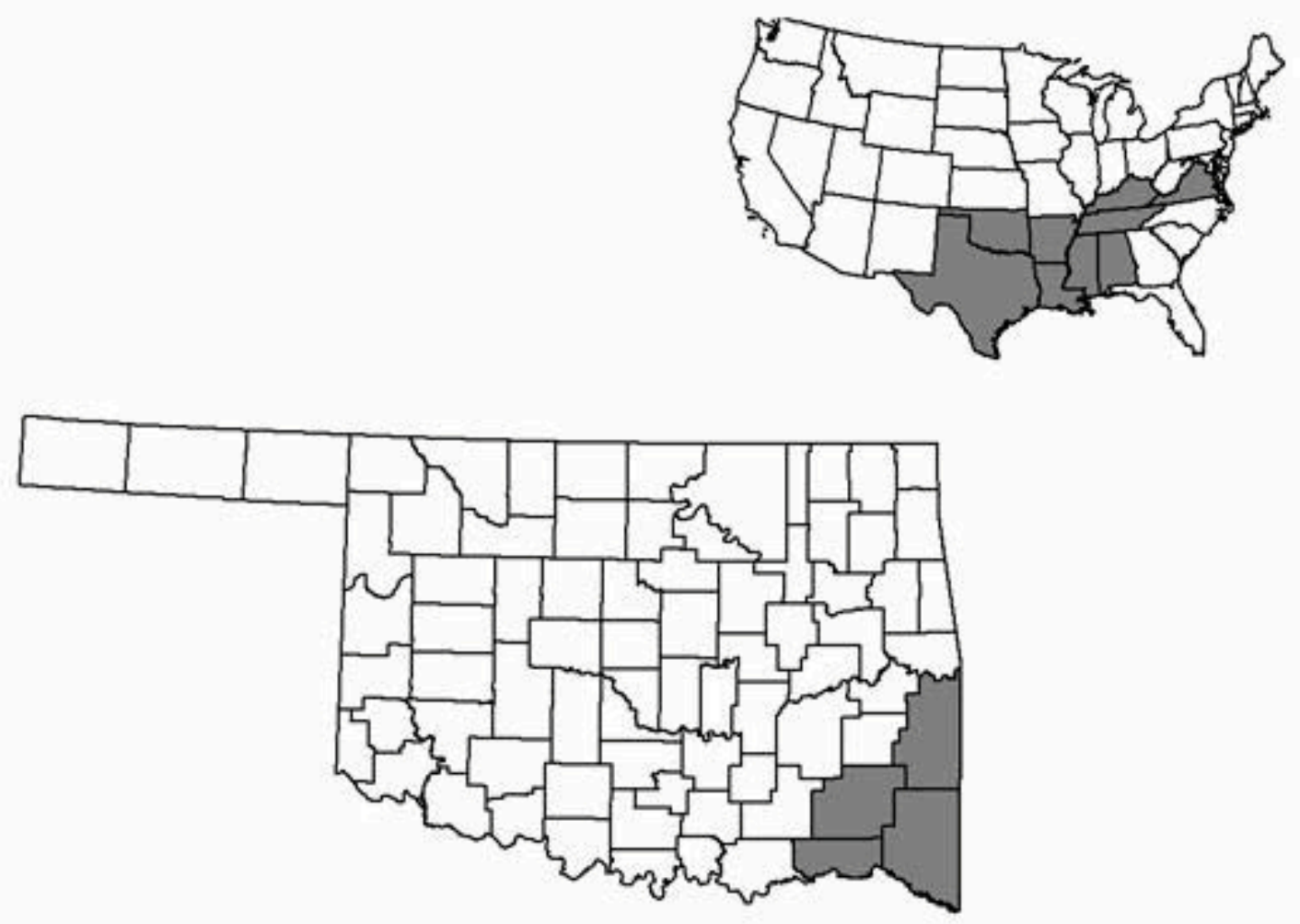

Figure National and state distribution of Cypripedium kentuckiense (Kentucky lady's slipper) (USDA 2002). 


\section{METHODS}

Sites visited in this study were obtained the ONHI, which maintains a spatial database of rare species locations. Each site was visited from late April to early May, the peak blooming period, in the springs of 2001 and 2002. All sites were thoroughly searched in an attempt to relocate previously documented $C$. kentuckiense populations. If a population was not found at a site, the absence was noted and no further data were collected. If a population was found, then several ecological variables were measured. These data were collected to quantitatively characterize the habitat of $C$. kentuckiense and provide information that can be utilized in future attempts to locate new populations.

Population and habitat data were collected from a quadrat encompassing all C. kentuckiense stems. The minimal quadrat size used was $1.0 \mathrm{~m}$ by $1.0 \mathrm{~m}$. If the population occupied a larger area, additional $1 \mathrm{~m}^{2}$ quadrats were added until the total population was within a sampling grid. Once the sampling grid was established, percent cover of $C$. kentuckiense was visually estimated in intervals of $5 \%$ and the number of stems counted. The numbers of flowering, fruiting (mature and immature), immature stems, and senescent stems were also recorded.

Habitat data consisted of biotic and abiotic factors. First, each species in the sampling grid was recorded. Two types of data were then collected for these associated species. First, percent cover was estimated for each understory species (including woody plants under $2 \mathrm{~cm}$ diameter) in increments of $5 \%$. If only a single stem of a species was present, it was given a value of $1 \%$. Second, the diameter-atbreast height $(\mathrm{DBH})$ was measured for all woody plants $>2 \mathrm{~cm}$ diameter. Basal area for canopy species was calculated following Wegner
(1984). Once these data were collected, a spherical densitometer was used to measure canopy closure. Soil depth was measured using an incremental probe. Finally, Universal Transmercator coordinates were recorded using a Garmin 3+ Global Positioning System (GPS) unit in order to resolve ambiguities in written location information. However, these data are not presented here because C. kentuckiense is a species of conservation concern.

\section{RESULTS AND DISCUSSION}

A total of twelve sites were surveyed in 2001 and 2002 for populations of $C$. kentuckiense; two in Choctaw County, six in LeFlore county, three in McCurtain County, and one in Pushmataha County. The persistence of three populations was verified. Five populations could not be revisited due to insufficient location information. Four populations had been destroyed by timber harvest or conversion to unsuitable habitat for C. kentuckiense. No new sites were located.

The first population was verified on 7 May 2001. Thirteen stems of C. Kentuckiense were counted, two of which were in flower. This population occurred in a mesic floodplain forest with 78\% canopy closure. There were 21 associated plant species present. The most abundant were Lindera benzoin (30\% cover), Thalictrum dascycarpum (15\%), Podophyllum peltatum (10\%), and Toxicodendron radicans $(10 \%)$ (Table 1$)$. The canopy was composed exclusively of Ilex opaca, a common bottomland species in southeast Oklahoma (Hoagland et al., 1996). The low diversity of woody plants over $2 \mathrm{~cm}$ DBH (Table 2) and relatively open canopy $(78 \%)$ indicate the second growth character of this forest. Soil depth was equivalent for all sites.

This population was first reported in 1982, at which time 35 stems were recorded,

Buthod, A.K. and Hoagland, B.W. 
but no observations were made regarding phenological state. The site was visited again in 1984 and 30 plants were recorded. Additional surveys were conducted in 1985 (23 plants located, two flowering), 1988 (13 plants; phenology not recorded), 1990 (less than 20 plants present, phenology not recorded), 1991 (21 plants, four in flower), 1993 (12 plants, two in flower), and 1996 (no plants located).

A second population was verified on 8 May 2001. Nine broadly dispersed stems of C. kentuckiense were present. Individuals in this population were widely dispersed. Eleven associated species were present, of which the most common were Parthenocissus quinquefolia (20\% cover), Panicum sp. (10\%), and Podophyllum peltatum (10\%) (See Table 1). The canopy was relatively dense $(87.3 \%)$ and consisted of eight species. The highest basal areas were recorded for Quercus shumardii and Carpinus caroliniana, both mesic species (Little 1981).

This population has been visited repeatedly since its discovery in 1988 (two plants, phenological stage not recorded), 1991 (nine plants flowering, two in fruit, and four sterile), 1992 (>10 plants with immature fruit), 1993 (11 plants, nine in flower), and 1996 (11 plants in vegetative condition).

A third population was verified on 8 May 2001. The population consisted of one flowering stem growing on a forested floodplain. Population and associated species data were collected from the $1.0 \mathrm{~m}^{2}$ plot. There were 21 associated plant species at this site (See Table 1). The most abundant were Panicum sp. (25\% cover), Parthenocissus quinquefolia (5\%), and Arisaema dracontium $(5 \%)$. Although the canopy density is highest for this plot, there were no canopy trees within the sample plot, therefore no basal area value could be calculated.
Previous surveys of the site were conducted in 1993 (one flowering stem recorded) and 1996 (one vegetative stem recorded).

Because of the limited number of sites sampled, a quantitative analysis of habitat structure is not possible.

\section{CONCLUSIONS}

Based upon this research, we conclude C. kentuckiense in Oklahoma is in jeopardy. Magrath (2001) had also stated that $C$. kentuckiense populations were in decline in Oklahoma. The populations that were relocated numbered fewer than 20 total stems and all showed a dramatic decline in population size relative to previous surveys. The primary threats to C. kentuckiense in Oklahoma are anthropogenic. Most populations of $C$. kentuckiense are located in areas of active timber harvesting, which present both direct and indirect threats. The most likely direct threat is destruction of a population by timber harvesting equipment. Indirect threats include road construction and structural alteration of adjacent forest stands. These reduce forest canopy cover, thus increasing the amount of light reaching the forest floor and allowing the introduction of invasive species. Since $C$. kentuckiense is difficult to propagate, it is frequently collected in the wild for the nursery trade. The construction of logging roads increases access to collectors. In addition, road construction itself can result in the destruction of a population.

Indirect threats to small, isolated populations included reduced genetic variability compared to large, contiguous populations, and the inability of pollinators to locate widely dispersed populations or those on the edge of a species range. In this regard, it is noteworthy that very few mature fruits or seedlings were documented in the populations reported here.

Nevertheless, additional populations of 
C. kentuckiense may be found in Oklahoma, with efforts focused on the Oachita National Forest (ONF) in LeFlore and McCurtain Counties. In Arkansas several new populations have been found on the ONF. Some populations consisted of 100 individuals or more (Hooks 2000). Populations located on the ONF are afforded a higher degree of protection and monitoring than those on private land. In addition, seep, spring, and riparian habitats are protected from timber extraction on Forest Service land. Thus, further exploration for populations of $C$. kentuckiense within Oklahoma is recommended. Likewise, sites known to have harbored populations of $C$. kentuckiense should be verified regularly.

\section{ACKNOWLEDGMENTS}

This project was funded by a grant from the United States Fish and Wildlife Service. We thank Newell McCarty for assistance in the field in 2001.

\section{LITERATURE CITED}

Alabama Natural Heritage Program. 1996. Plants list (on line). Available at: www.natureserve.org/nhp/us/al/pl ants.html. (Accessed 1 March 2004)

Arkansas Natural Heritage Commission. 2001. Plants of special concern (online). Available at: www.naturalheritage.org/ publications/rare. (Accessed 1 March2004).

Atwood, John T. 1984. In defense of Cypripedium kentuckiense C. F. Reed. American Orchid Society Bulletin 53(8): 835-841.

Atwood, John T. The range of Cypripedium kentuckiense. American Orchid Society Bulletin 54(10): 1197-1199.
Case, Martha A., Henry T. Mlodozeniec, Lisa E. Wallace, and Troy W. Weldy. 1998. Conservation genetics and taxonomic status of the rare Kentucky lady's slipper: Cypripedium kentuckiense (Orchidaceae). American Journal of Botany 85(12): 1779-1786.

Department of the Interior. 1993. Federal Register, Part IV 58(188): 51160.

Groves, C.R., M.L. Klein, and T.F. Breden. 1995. Natural Heritage Programs: public-private partnerships for biodiversity conservation. Wildlife Society Bulletin 23:784-790.

Hoagland, B.W., L.R. Sorrels and S.M. Glenn. 1996. Woody species composition of floodplain forests of the Little River, McCurtain and LeFlore Counties, Oklahoma. Proceedings of the Oklahoma Academy of Science 76:23-26.

Hoagland B.W., A.K. Buthod, I.H. Butler, P.H.C. Crawford, A.H. Udasi, W.J. Elisens, and R.J. Tyrl 2004. Oklahoma Vascular Plants Database (on line). Oklahoma Biological Survey, University of Oklahoma, Norman. Available at: www.biosurvey.ou.edu. (accessed 1 March 2004).

Hooks, Susan. 2000. Conservation Assessment for Cypripedium kentuckiense Reed on the Ouachita and Ozark St. Francis National Forests. United States Forest Service, Hot Springs, Arkansas.

Kentucky State Nature Preserves Commission. 2001. Endangered, threatened, special concern, and historic plants and animals of Kentucky (on line). Available at: www.kynaturepreserves.org/reports .html. (Accessed 1 March 2004).

Little, E.L. 1981. Forest trees of Oklahoma. Oklahoma Forestry Division, Publication number 1, Oklahoma City.

Buthod, A.K. and Hoagland, B.W. 
Louisiana Natural Heritage Programs. 2002. Rare plant species of Louisiana (on line). Available at: www.wlf.state.la.us. (Accessed 1 March 2004).

Magrath, L.K. 2001. Native Orchids of Oklahoma. Oklahoma Native Plant Record 1 (1):39-66.

Mississippi Museum of Natural Science. 2002. Natural heritage inventory plant database (on line). Available at: www.mdwfp.com/ museum $/ \mathrm{html} /$ research/plant db.a sp. (Accessed 1 March 2002).

NatureServe. 2004. NatureServe Explorer: An online encyclopedia of life (on line). Available at: www.natureserve.org/explorer. (Accessed 1 March 2004)

Oklahoma Natural Heritage Inventory. 2001. ONHI guide to status and rarity ranking codes (on line). Available at: www. biosurvey.ou.edu/heritage/publicat. html. (Accessed 1 March 2004).

Oklahoma Natural Heritage Inventory. 2001. ONHI working list of rare Oklahoma plants (on line). Available at: www.biosurvey.ou.edu/heritage/ publicat.html. (Accessed 1 March 2004).

Reed, Clyde F. 1982. Cypripedium kentuckiense Reed, a new species of orchid in Kentucky. Phytologia 50(4): 286-288.
Tennessee Department of Environment and Conservation. 2001. Rare and endangered plant list of Tennessee (on line). Available at: www.state.tn.us/environment/natur al.htm. (Accessed 1 March 2004).

Texas Conservation Data Center. 2001. Plant tracking list (on line). Available at:

nature.org/wherewework/northam erica/states/ texas/science/art6069.html. (Accessed 1 March 2004).

USDA, NRCS. 2002. The PLANTS database, version 3.5 (on line). Available at: http://plants.usda.gov/. National Plant Data Center, Baton Rouge, Louisiana. (Accessed 1 March 2004).

Virginia Natural Heritage Program. 2002. Rare plants (on line). Available at: www.dcr.state.va.us/dnh/nhrinfo.ht m (Accessed 1 March 2002).

Weldy, Troy W., Henry T. Mlodozeniec, Lisa E. Wallace, and Martha A. Case. 1996. The current status of Cypripedium kentuckiense (Orchidaceae) including a morphological analysis of a newly discovered population in eastern Virginia. Sida 17(2): 423-435.

Wenger, K.F. 1984. Forestry handbook. John Wiley and Son, New York 
Table 1 Percent cover data for associated herbaceous species and woody species $<2 \mathrm{~cm} \mathrm{DBH}$ at Cypripedium kentuckense sites in southeastern Oklahoma.

\begin{tabular}{|c|c|c|c|c|c|c|c|}
\hline & Site 1 & 2 & 3 & Site & 1 & 2 & 3 \\
\hline Acer rubrum & 1 & 0 & 1 & Podophyllum peltatum & 10 & 0 & 10 \\
\hline Allium canadense & 0 & 0 & 1 & Polystichum acrostichoides & 5 & 0 & 5 \\
\hline Arisaema dracontium & 0 & 5 & 0 & Potentilla spp. & 1 & 0 & 0 \\
\hline Campsis radicans & 1 & 0 & 0 & Prunus spp. & 1 & 0 & 0 \\
\hline Carex spp. & 1 & 1 & 0 & Ribes sp. & 0 & 1 & 0 \\
\hline Cercis canadensis & 0 & 0 & 1 & Quercus alba & 0 & 0 & 5 \\
\hline Cypripedium kentuckense & 5 & 5 & 1 & Salvia lyrata & 0 & 1 & 0 \\
\hline Euonymous americanus & 1 & 0 & 0 & Sassafras albidum & 1 & 0 & 0 \\
\hline Galium sp. & 1 & 0 & 0 & Senecio sp. & 0 & 5 & 1 \\
\hline Ilex opaca & 1 & 0 & 0 & Smilicina racemosa & 0 & 0 & 1 \\
\hline Impatiens capensis & 0 & 0 & 1 & Smilax glauca & 1 & 0 & 5 \\
\hline Krigia sp. & 0 & 1 & 0 & Thalictrum arkansanum & 0 & 1 & 0 \\
\hline Lindernia benzoin & 30 & 0 & 0 & Thalictrum dasycarpum & 15 & 0 & 5 \\
\hline Lysimachia quadrifolia & 0 & 0 & 5 & Toxicodendron radicans & 10 & 1 & 10 \\
\hline Mitchella repens & 1 & 0 & 0 & Ulmus alata & 0 & 0 & 1 \\
\hline Monarda virgatum & 0 & 0 & 1 & Viburnum rufidulum & 1 & 0 & 1 \\
\hline Ostrya virginiana & 1 & 0 & 1 & Viola sp. & 1 & 0 & 0 \\
\hline Oxalis violacea & 0 & 1 & 0 & Viola pedata & 1 & 0 & 1 \\
\hline Panicum spp. & 0 & 25 & 10 & Totals & 91 & 52 & 88 \\
\hline Parthenocissus quinquefolia & 1 & 5 & 20 & Overstory canopy $\%$ coverage & 78 & 87.5 & 93.8 \\
\hline Phacelia sp. & 0 & 0 & 1 & Soil depth (cm) & 30 & 30 & 30 \\
\hline
\end{tabular}

Buthod, A.K. and Hoagland, B.W. 
Table 2 Basal area $\left(\mathrm{cm}^{2} / \mathrm{m}^{2}\right)$ for woody species found in Cypridedium kentuckense. No woody plants occurred in the plot at site 3

\begin{tabular}{lcclcc}
\hline & Site & 1 & 2 & & Site 1 \\
\hline Alnus serrulata & 0 & 0.07 & Ilex opaca & 8.56 & 0 \\
Carpinus caroliniana & 0 & 8.0 & Liquidambar styraciflua & 0 & 8.0 \\
Cornus florida & 0 & 2.8 & Ostrya virginiana & 0 & 6.6 \\
Fraxinus pensylvanica & 0 & 0.29 & Quercus shumardii & 0 & 10.8
\end{tabular}

Buthod, A.K. and Hoagland, B.W. 


\title{
Common Lawn and Garden Mushrooms of Central Oklahoma
}

\author{
Clark L. Ovrebo \\ Department of Biology \\ University of Central Oklahoma \\ Edmond, OK 73034
}

Mushrooms are often abundant in lawns and gardens after periods of wet weather. This article presents photographs of some of the more common mushrooms the author has encountered in Central Oklahoma over the past fifteen years.

\section{INTRODUCTION}

A mushroom is the fruiting body of a fungus. The body of the fungus, termed mycelium, is found in soil, compost, wood chips, or logs. Rarely are mushroom species parasitic. The mycelium gets nutrition from breaking down the organic substrates (saprotrophic mode of nutrition). If the yard has oaks, hickories or pines, the fungus forms a mycorrhizal association with the tree roots, an association that is beneficial to both the fungus and the tree. Mycorrhizal fungi are most often found in forested areas, but may also occur in yards or city parks if their symbiotic trees are present. With the exception of Suillus brevipes, the fungi treated in this paper are saprotrophic.

The term "common," is not so easily defined. Some mushrooms appear every year after almost every rain, whereas others may appear only sporadically even after wet weather. Some fungi are abundant some years and not the next. Thus, the term "common" as used for fleshy fungi must remain vague. The time of year given for fruiting is also somewhat general and is intended to give an approximation of when the mushrooms fruit.

In addition to photos, a brief description is provided for each species. The intent is not to provide complete macroscopic and microscopic descriptions of the fungi, but rather, the salient morphological features. To be sure of identification, it may be necessary to use microscopic examination and consult field guides or technical literature. Spore print color is presented for some species because determining spore color is often the first step in identifying a mushroom. A spore print is made by cutting off the stipe and placing the pileus with lamellae side down on a piece of white paper. This set-up is covered and left overnight.

Advice is not provided about which lawn mushrooms are edible. Great care must be taken to be absolutely sure of a mushroom's identity. Only after becoming sufficiently familiar with mushroom characters and their identifying features can one be certain of the identification, and only then can the determination about edibility be made.

Many field guides are helpful and provide additional information. A listing of some field guides that are useful for Oklahoma is found at the end of the article.

\section{Agaricales}

Fungi in this order comprise one of the more common groups of lawn fungi and include what are called "gilled mushrooms" and "boletes". The parts of a gilled mushroom are the pileus (cap), stipe (stem), and lamellae (gills). A bolete has tubes that end in pores instead of lamellae. The spores are produced on the lamellae or tubes, forcibly discharged, and are dispersed by air currents.

Ovrebo, C.L.

https://doi.org/10.22488/okstate.17.100032 


\section{Chlorophyllum molybdites}

Scattered, on lawns or pastures, sometimes in fairy rings or arcs (Figure 1), late spring through mid-fall. Chlorophyllum molybdites is one of the most common larger mushrooms occurring on lawns. It is recognized by the rather large fruiting bodies, whitish pileus surface with tan scales near or on the center, green lamellae and by the partial veil which leaves an annulus on the stipe (Figure 2). The lamellae are off-white when young and become green at maturity, and are free (not attached to the stipe). The spore deposit is green. Chlorophyllum molybdites is the leading cause of mushroom poisoning in the United States. It causes severe gastrointestinal upset with recovery after several hours. It is especially toxic to young children and people already compromised by health problems.

\section{Amanita thiersii}

Scattered, on lawns, sometimes in fairy rings or arcs (Figure 3), summer and fall. Amanita thiersii is another of the larger mushrooms occurring on lawns and may be in fruit at the same time as C.molybdites. The pileus and stipe are white and the lamellae light cream-colored. Young fruiting bodies are covered with a flocculent coating that may remain throughout maturation (Figure 4). The covering is easily removed when touched and may be washed away with rain. The spore print is white. This species belongs in the genus that contains some of the most deadly poisonous mushrooms. Though little is known about the toxicity or edibility of $A$. thiersii, it is probably poisonous.

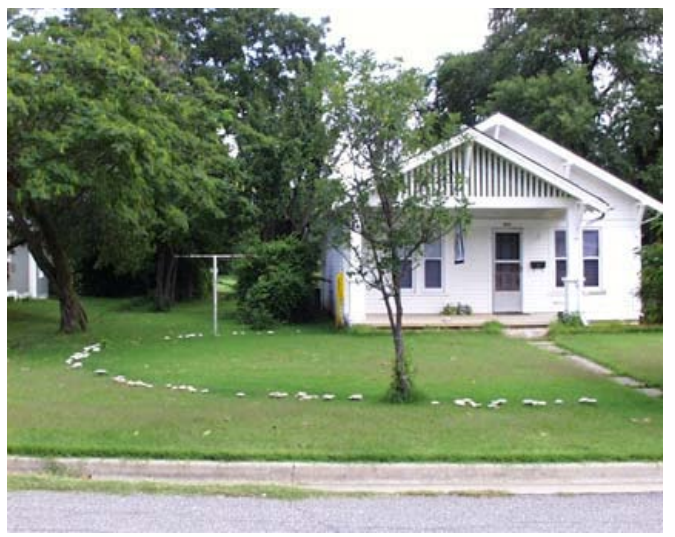

Figure 1 Chlorophyllum molybdites, fairy ring.

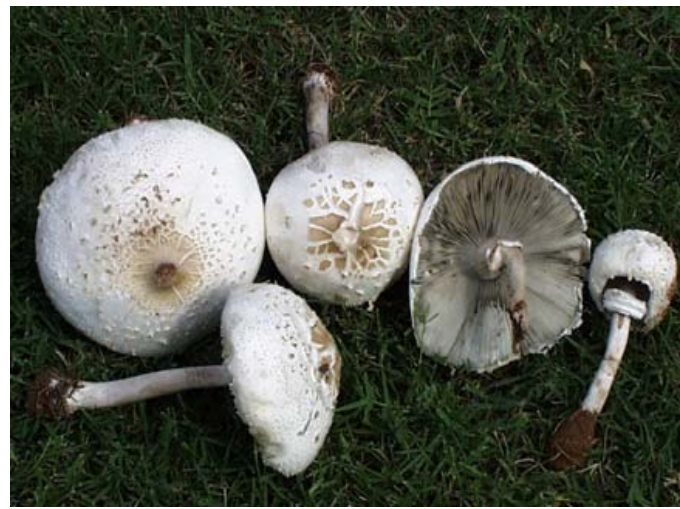

Figure 2 Chlorophyllum molybdites, fruiting bodies.

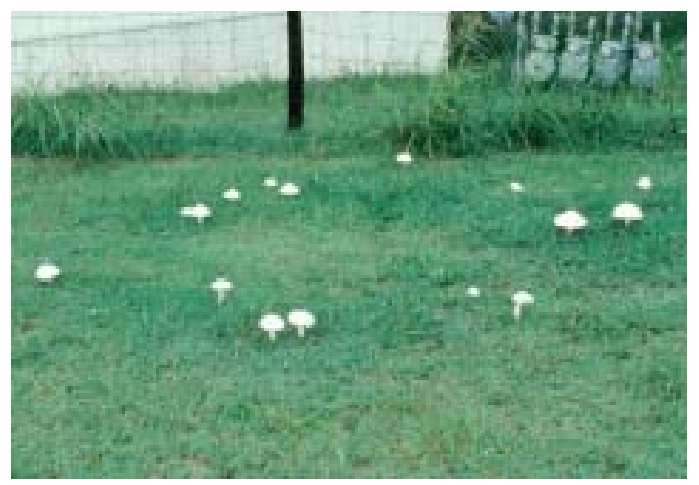

Figure 3 Amanita thiersii, fairy ring.

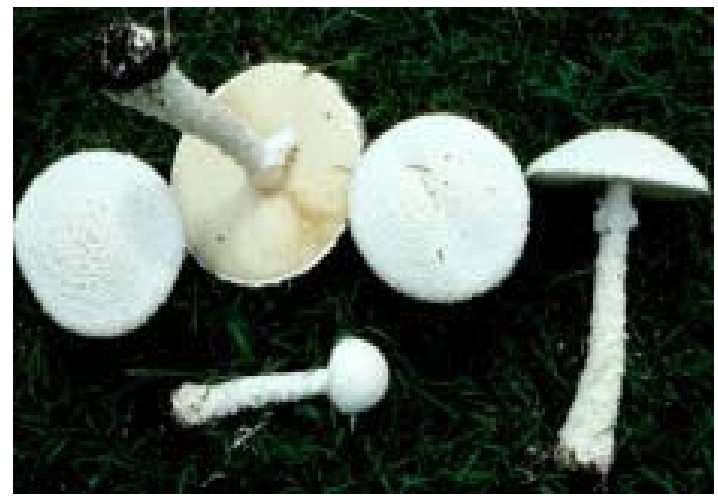

Figure 4 Amanita thiersii, fruiting bodies. 
Marasmius oreades (fairy ring mushroom)

Scattered, on lawns, occasionally in arcs or fairy rings (Figure 5), summer to late fall. Marasmius oreades is a small, thin-statured mushroom with the pileus reaching at most 3-4 $\mathrm{cm}$ in diameter. The pileus is smooth, off-white with the center often light $\tan$ (Figure 6). The buttons can be light brown overall. The lamellae are rather distantly spaced and off-white. The stipe is also off-white and lacks an annulus. The spore print is white.

\section{Coprinus comatus (shaggy mane)}

Scattered, on lawns, late summer through late fall. Coprinus comatus is easily recognized by the rather tall fruiting bodies that have a vertically elongated pileus. The pileus of the buttons is elliptical. The pileus has a shaggy surface and is off-white although the top can be $\tan$ (Figures 7 and 8). The lamellae are off-white when young and black at maturity. The genus is characterized by the fact that the mushrooms deliquesce (auto-digest). Beginning at the margin, the pileus begins to liquefy and the process continues toward the top-center of the pileus (Figure 9). Often only the stipe remains.

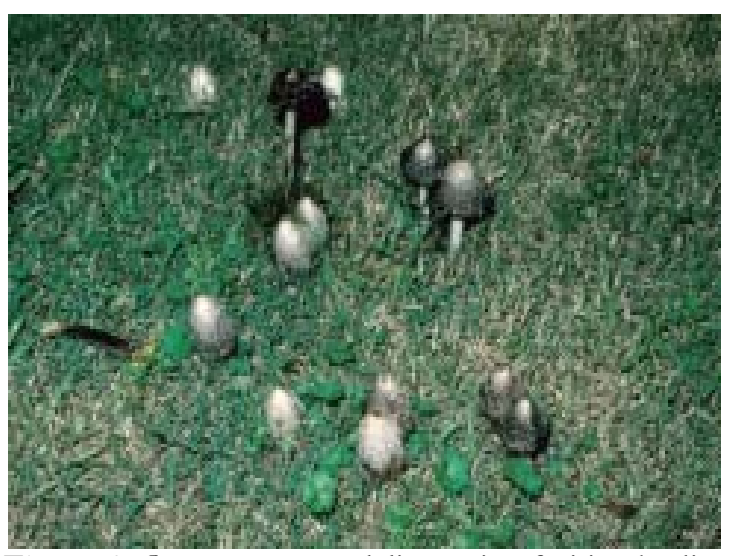

Figure 9 Coprinus comatus, deliquescing fruiting bodies

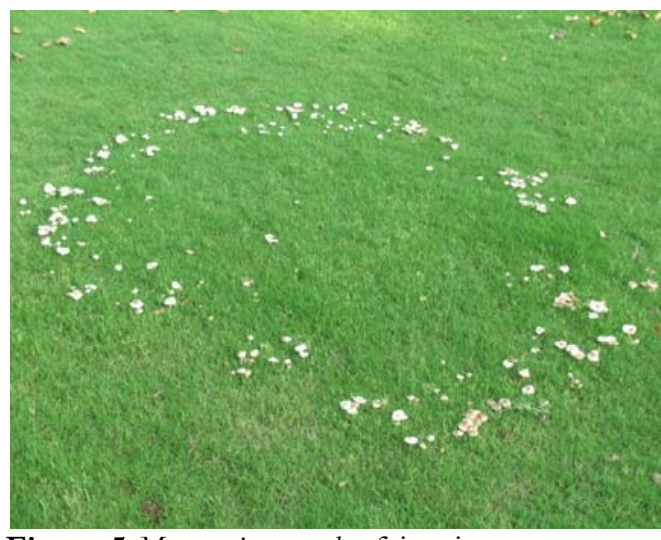

Figure 5 Marasmius oreades, fairy ring.

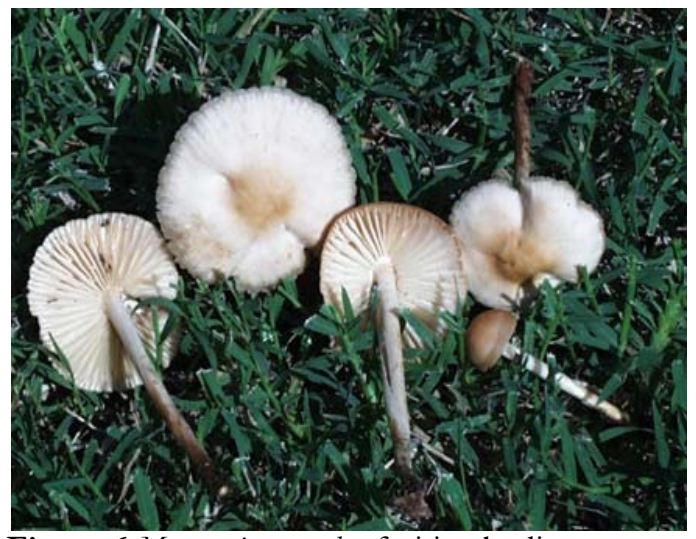

Figure 6 Marasmius oreades, fruiting bodies.

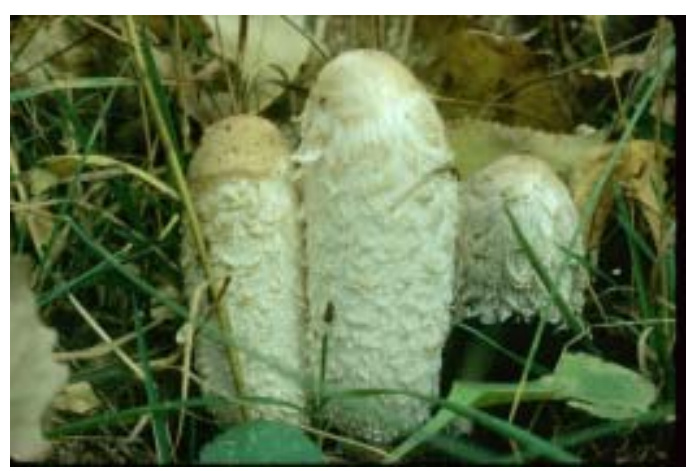

Figure 7 Coprinus comatus, fruiting bodies showing shaggy surface.

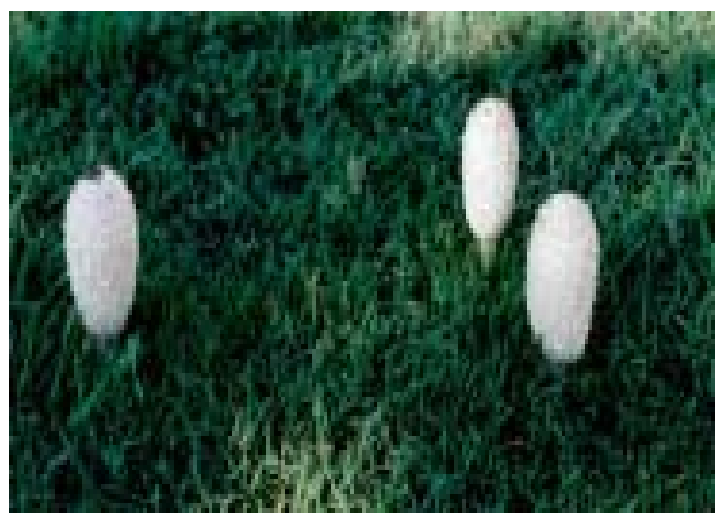

Figure 8 Coprinus comatus, mature fruiting bodies.

Ovrebo, C.L. 


\section{Coprinopsis variegata}

Scattered to clustered, on lawns, but attached to buried wood, or on stumps, summer. Coprinopsis variegata appears in clusters on lawns but is actually growing from buried wood such as the remains of a stump. The pileus is off-white to grayish brown to gray, and has scales or patches (Figures 10 and 11). Like Coprinus comatus, the fruiting bodies deliquesce. This species was formerly placed in Coprinus.

\section{Conocybe lactea}

Scattered, on lawns, early summer. Conocybe lactea has small fragile fruiting bodies that are evident in the morning but wither away as the heat of the day sets in. The pileus is whitish to light tan and rounded-conic. The lamellae are light cinnamon-brown (Figure 12).

\section{Parasola plicatilis}

Scattered, on lawns, early summer. Fruiting bodies of Parasola plicatilis are small and thin-statured (Figure 13). The pileus is translucent-gray and plicate (grooved). The lamellae are black. This species is most noticeable in the morning. In sunlight it quickly dries and disappears. This species was also formerly placed in Coprinus.

\section{Agaricus campestris (meadow mushroom)}

Scattered, on lawns and pastures, summer to early fall. Agaricus campestris is characterized by the whitish pileus and stipe, annulate stipe and by the dark brown, free lamellae (Figure 14). The lamellae start out light pink in the button stage and become dark brown as the spores mature. The spore print is dark brown. This species is in the same genus as the cultivated button mushroom that is available fresh or canned in grocery stores.

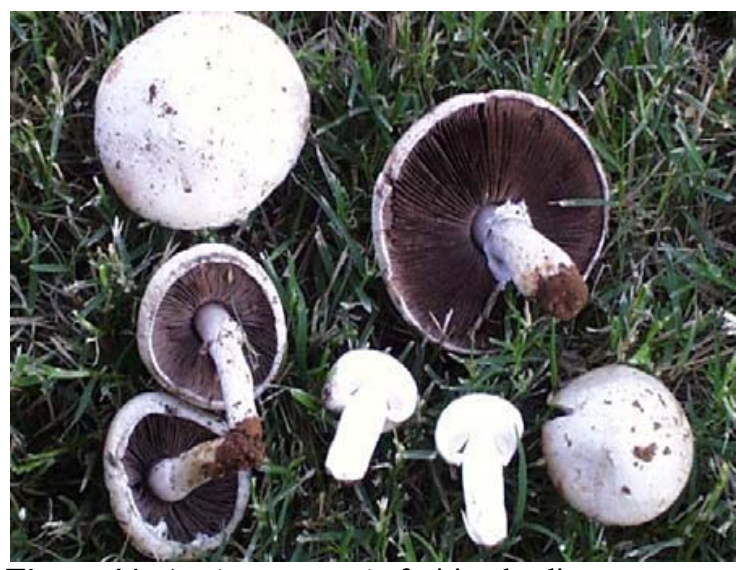

Figure 14 Agaricus campestris, fruiting bodies.

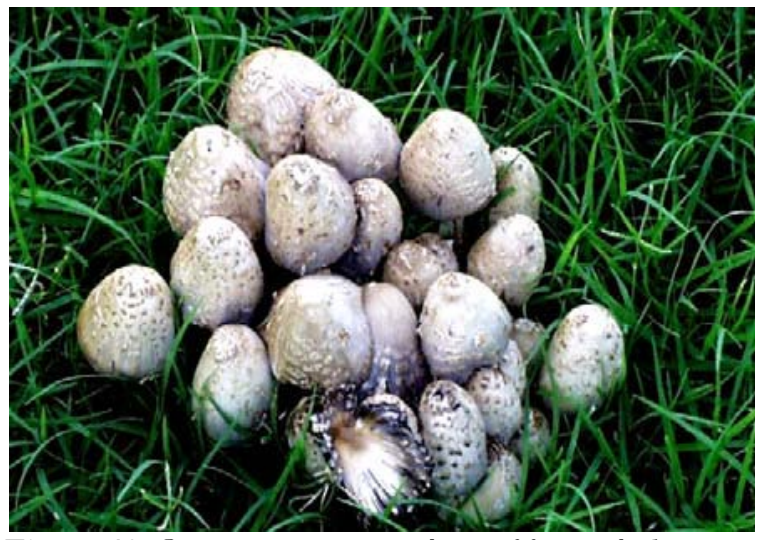

Figure 10 Coprinopsis variegata, cluster of fruiting bodies.

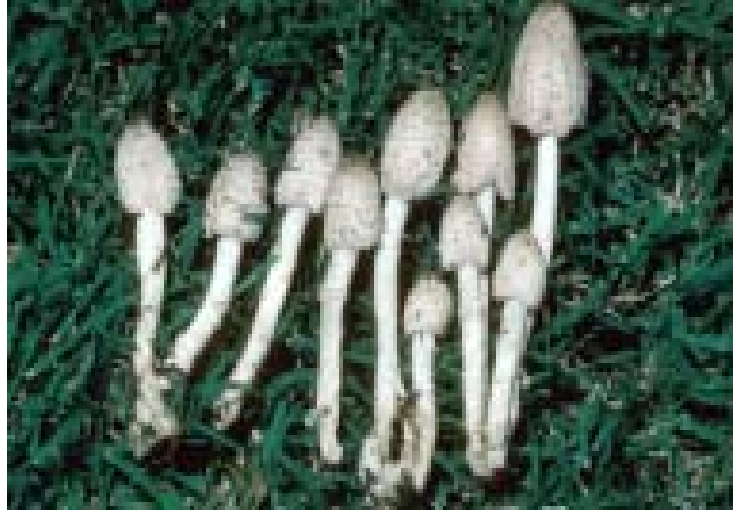

Figure 11 Coprinopsis variegata, fruiting bodies.

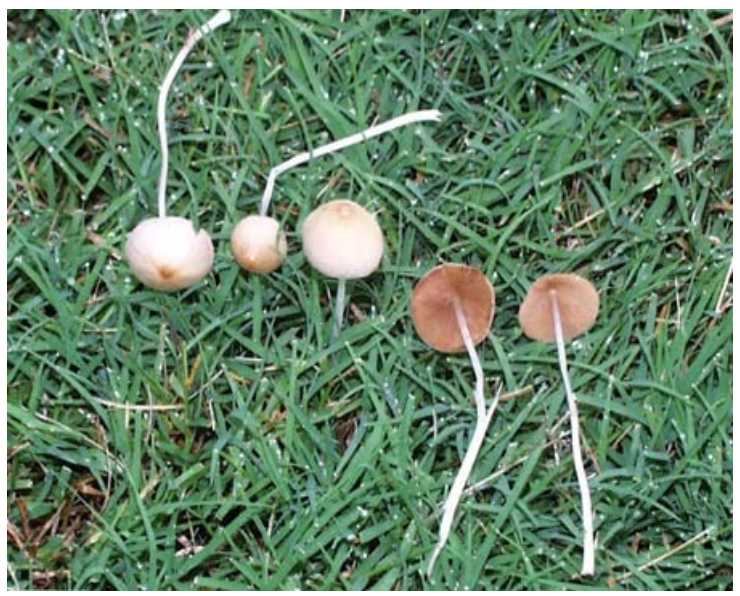

Figure 12 Conocybe lactea, fruiting bodies.

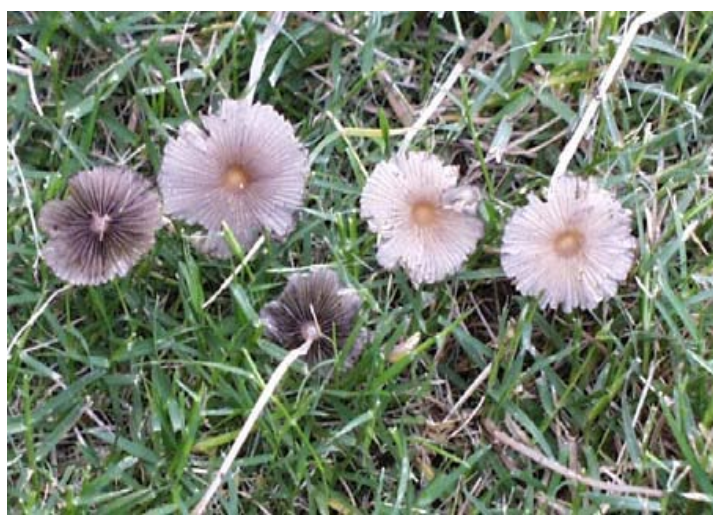

Figure 13 Parasola plicatilis, fruiting bodies.

Ovrebo, C.L. 


\section{Leucoagaricus naucinus}

Scattered on lawns, fall. Leucoagaricus naucinus is a medium-sized mushroom and is creamy white overall. The pileus is smooth, the lamellae are free and the stipe has an annulus (Figure 15). The spore print is white. This species is not edible and causes mild to severe gastric upset. There is no cup at the base as with Amanita virosa, which is similar in coloration but occurs in forests and is deadly poisonous.

\section{Pluteus petasatus}

On dead wood or wood chips, in clusters of two or three, summer. The pileus of Pluteus petasatus is off-white to light tan with the center becoming brown. The center of the pileus develops cracks in age. The lamellae are pinkish tan and free (Figure 16). The spore print is pinkish tan.

\section{Clitocybe tarda}

Scattered, on lawns, generally in clusters, fall. Clitocybe tarda is characterized by the smooth, violet-purple pileus (Figure 17). The lamellae and stipe are pigmented similarly but generally lighter. With age considerable fading of the pileus may occur. The spore print is very pale pinkish buff.

\section{Suillus brevipes}

Scattered, on soil or lawns underneath Pinus spp., late fall. Suillus brevipes has tubes and pores rather than lamellae. The pileus is brown and slimy when fresh and in age the color fades to yellowish tan and the surface may dry. The tubes and pores are yellow when young and become more olive-tinged in age (Figure 18). Suillus brevipes forms a mycorrhizal association with Pinus.

Ovrebo, C.L.

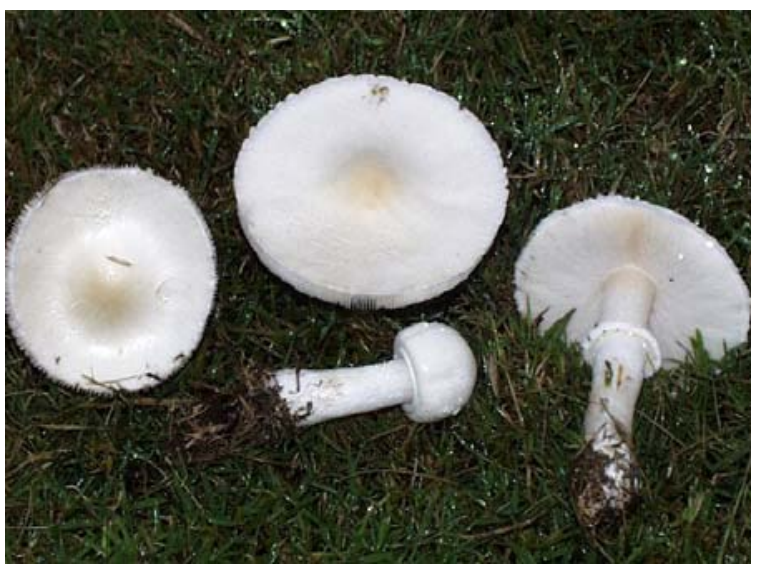

Figure 15 Lencoagaricus naucinus, fruiting bodies.

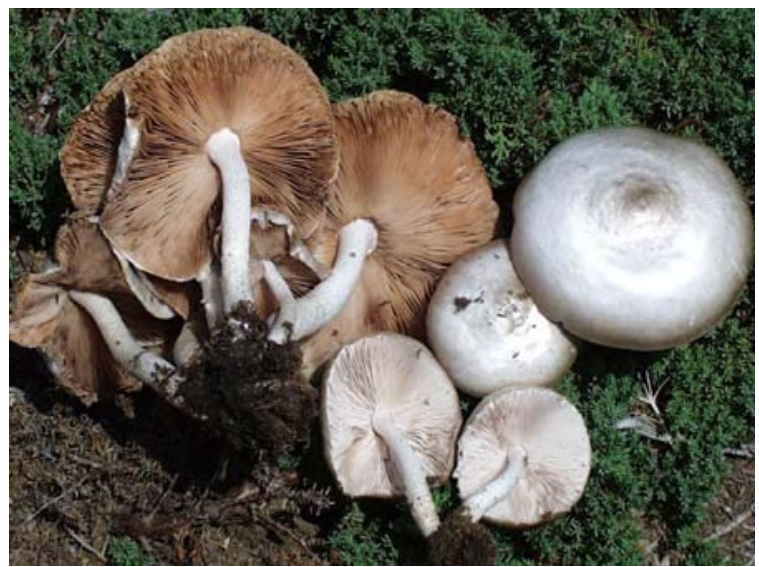

Figure 16 Pluteus petasatus, fruiting bodies.

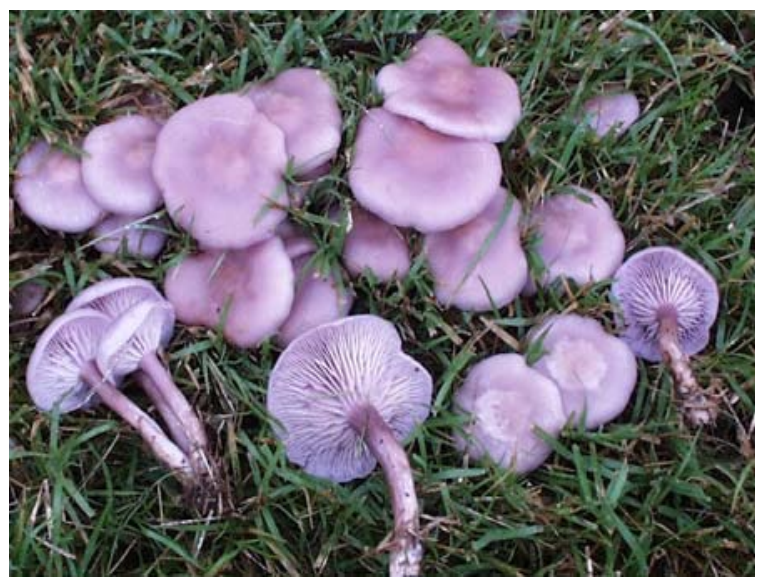

Figure 17 Clitocybe tarda, fruiting bodies.

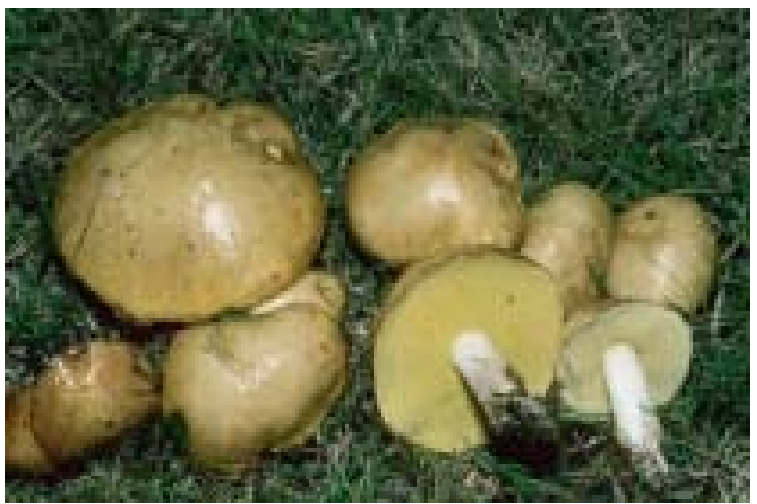

Figure 18 Suillus brevipes, fruiting bodies. 


\section{Order Phallales (Stinkhorns)}

The stinkhorns comprise a most interesting group of fleshy fungi. Rather than using air currents for spore dispersal, the spores are borne on a sticky mass called the gleba, which has a repulsive odor. For the species discussed here, the gleba is formed at the tip of the mushroom and is dark olive to nearly black. The odor attracts flies and other insects which are the agents of spore dispersal. The fungi start out as "buttons" completely encapsulated by an outer membrane (universal veil). When the mushroom bursts out of the button, a cup (volva) is left at the base. A section through the button reveals the immature fruiting body. All species of Phallus have a phallus-shaped fruiting body. Various stages of development can be seen in Figures 19-21, 23.

\section{Phallus ravenelii}

Scattered on lawns, soil or wood mulch, summer to fall. This species is identical in stature to Phallus hadriani, but the gleba is light gray, nearly smooth, and not pitted. Note the flies on the gleba of Figure 21.

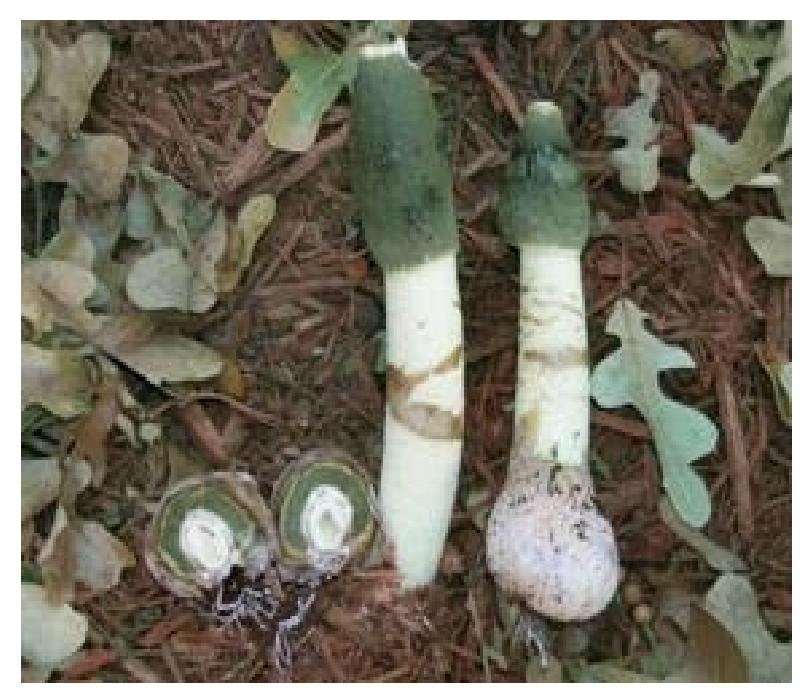

Figure 21 Phallus ravenelii, longitudinally sectioned button and fruiting bodies.

\section{Phallus hadriani}

Scattered, on lawns, soil or wood mulch, summer to fall. Phallus hadriani is distinguished by the pitted gleba (Figure 19). The surface of the volva is pink colored in this species. Phallus impudicus is identical in appearance but the outer surface of the volva is white.

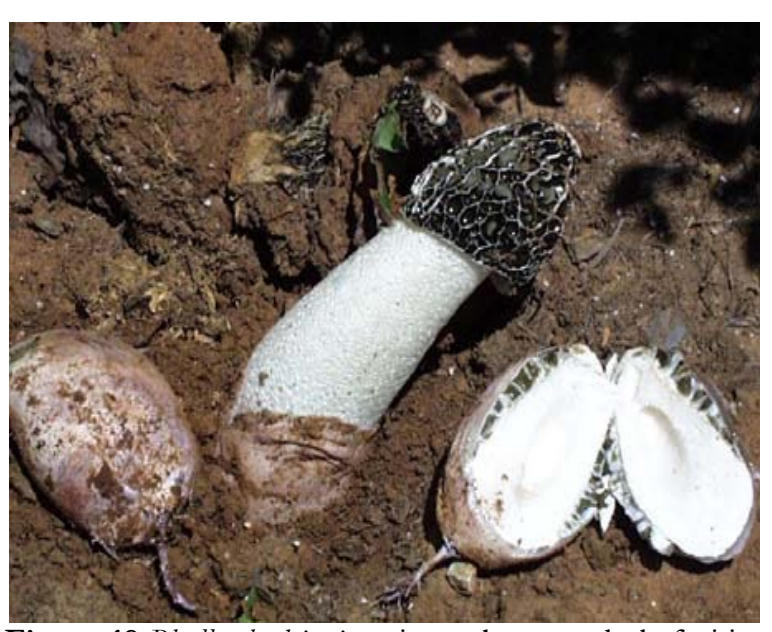

Figure 19 Phallus hadriani, an intact button whole fruiting body, and a longitudinally sectioned button.

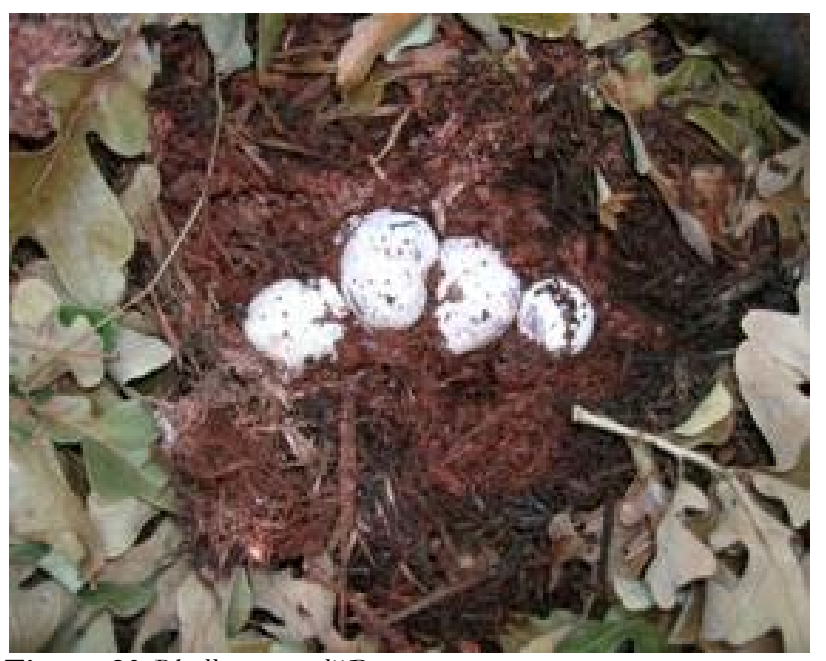

Figure 20 Phallus ravenelii,Buttons

Ovrebo, C.L. 


\section{Phallus rubicundus}

Scattered on lawns, summer. Phallus rubicundus also has the phalloid stature but the stipe is orange (Figure 22).

\section{Lysurus periphragmoides}

Solitary or scattered on lawns, summer to late fall. Lysurus periphragmoides is another common stinkhorn. It is generally smaller than the Phallus species, has an orange stipe and the glebal head is different. The glebal head has a sterile orange lattice network with gleba in between the netted pattern (Figure 23). Some field guides refer to this species as Simblum sphaerocephalum.

\section{FIELD GUIDES}

Arora, David. 1986. Mushrooms

demystified. $2^{\text {nd }}$ ed. Ten Speed Press, Berkeley, CA.

Bessette, Alan E., David W. Fischer, and A. R. Bessette. 1997. Mushrooms of Northeastern North America. Syracuse University Press.

Horn, B., R. Kay, and D. Abel. 1993. A guide to Kansas mushrooms. University of Kansas Press.

Lincoff, Gary. 1981. Audubon field guide to North American mushrooms. Alfred Knopf, New York.

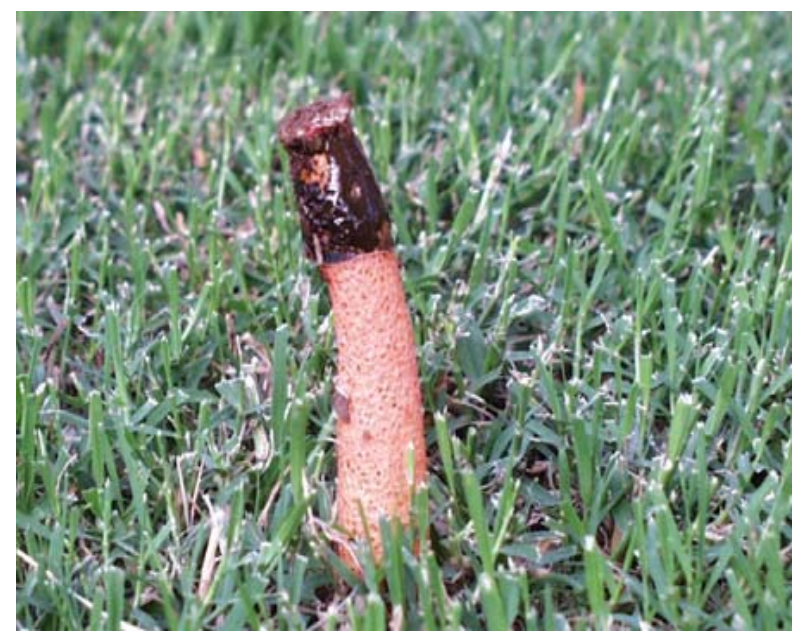

Figure 22 Phallus rubicundus, fruiting body.

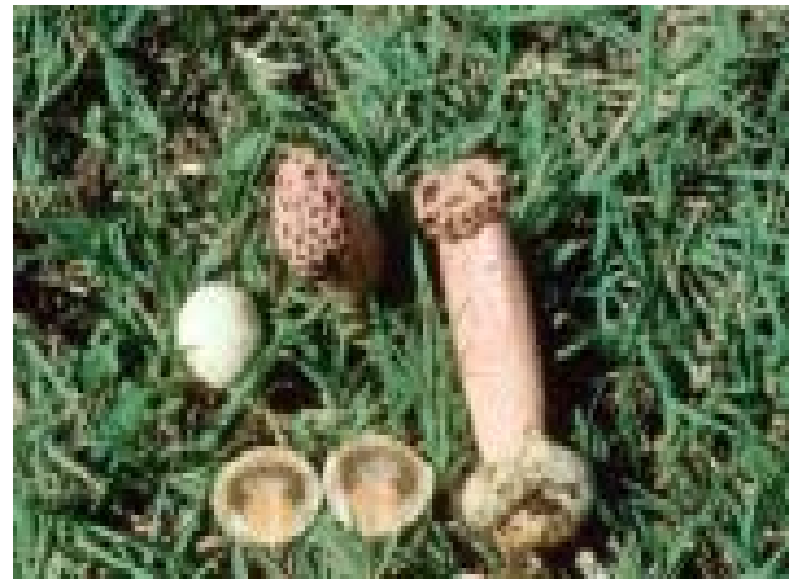

Figure 23 Lysurus periphragmoides, mature fruiting body with intact and longitudinally sectioned buttons.

Metzler, S., and V. Metzler. 1992. Texas Mushrooms. University of Texas Press, Austin.

Miller, O.K. 1977. Mushrooms of North America. Paperback ed. Dutton Publications. New York [out of print].

Smith, A. H., and Nancy S. Weber. 1981. The mushroom hunter's field guide. All colored revised ed. University of Michigan Press, Ann Arbor.

Weber, N. S., and A. H. Smith. 1985. A field guide to southern mushrooms. University of Michigan Press, Ann Arbor

Ovrebo, C.L. 


\title{
Critic's Choice Essay "SUPPORT YOUR LOCAL HERBARIUM"
}

\author{
Wayne J. Elisens
}

Are herbaria becoming threatened and endangered? Are natural science collections going extinct? These questions are being posed increasingly by amateur and professional biologists as we witness the closure, dispersion, and scaling back of natural history collections and cutbacks affecting curatorial personnel. Noteworthy examples include the elimination of several collections and removal of the herbarium curator at the University of Nebraska State Museum, the transfer of the herbarium collections from the University of Iowa to the herbarium of Iowa State University, and the narrow escape from closure for the University of Arkansas herbarium in Fayetteville. These and other events at various institutions have prompted several recent editorials in the science literature stating that the nation's natural history collections are "in crisis" (Dalton 2003, Gropp 2003, and Raven 2003). What about Oklahoma's herbaria? Should we be concerned about their health and well-being? What can we do? The first thing we can do as natural historians and concerned citizens is to dispel misinformation. For example, herbaria and other natural history collections should not be portrayed as the equivalent of a "stamp collection" (as I heard once from a botanical colleague), but rather as biological research collections. This latter phrase is the foundation for the acronym of the National Science Foundation program (the BRC program) that funds infrastructural improvement and computerization of natural history collections. A herbarium is more than just the physical collection of plants that have been pressed, dried, and stored in packets or mounted on paper of archival quality. Specimens include labels with critical information about the plant's identity, geographic location, ecological habitat, flowering time, and collecting history. Each specimen embodies the species' morphology (its phenotype) AND its genome (its genotype). In other words, each specimen represents valuable DATA and the entire collection should be viewed as a vast data and DNA bank. Oklahoma's herbaria serve as important resources for its citizens and as critical research tools for an international network of scientists, educators, resource managers, and amateur botanists (see Funk 2003).

Thirteen herbaria with combined holdings exceeding 450,000 specimens constitute the "Oklahoma herbarium community" (Table 1). Twelve of these herbaria are listed in Index Herbariorum (Holmgren et al 1990), which is the official international registry of herbaria compiled by the International Association for Plant Taxonomy and the New York Botanical Garden. Each herbarium has noteworthy regional, ecological, and taxonomic specializations. Despite the size and significance of the collections, most of Oklahoma's herbaria are inadequately supported, some have no "hard" budgetary support, most need facility upgrades, and the majority is maintained by the efforts of one or two individuals with limited help from students and a few volunteers. Faculty retirements, budget cuts, and personnel changes make some herbaria "vulnerable." To return to the opening question, some of Oklahoma's herbaria can be categorized as "endangered", because they meet the criterion of possible extinction in the foreseeable future. What is being done to ensure their survivability?

Oklahomans are fortunate to have a highly interactive network of plant taxonomists. More than in most states, plant taxonomists from across Oklahoma have an uncommon sense of collegiality and are collaborating to study the state's flora, to database label information from Oklahoma plant specimens, and to interact with the community of amateur botanists such as those in the ONPS, TNC, etc. Nine botanists representing seven institutions are working to complete a modern flora for the state - the Flora of Oklahoma project. Additionally, botanists at OU and OSU working with their colleagues in the Oklahoma herbarium community are recording label data from Oklahoma plant specimens for the Oklahoma V ascular Plants Database project. Both of these projects draw on collective knowledge, advance the study of Oklahoma's flora, share scientific expertise and resources, and promote the significance of the state's herbarium collections. Despite these positive developments, there are many areas where the public's help is needed to avoid extinctions. 
Herbaria need advocates in both the professional and public arenas. Just as individual curators must promote research and the importance of their collections to administrators and colleagues, amateur botanists both individually and collectively must elevate public awareness of the importance of herbaria. At the University of Arkansas, two events apparently impressed administrators and "saved" the herbarium from closure - the mass response from the professional botanical community and the widespread support throughout Arkansas from amateur botanists and natural historians. Some important lessons from the Arkansas case are the significance of outreach efforts and the reciprocal nature of herbarium activities. In Oklahoma, I am constantly impressed with the number of curators and professional biologists that maintain active public service involvement as officers and board members of organizations and through participation in lectures, field trips, workshops, and other functions. These activities result directly and indirectly from the presence of functioning herbaria located throughout the state and from the actions of knowledgeable professional staff.
In view of the "crisis" impacting natural history collections and herbaria nationwide, I urge amateur botanists to advocate for and to assist herbaria whenever possible. One mechanism to do this is to use the $\boldsymbol{P V C}$ model: Participate in sponsored activities, Volunteer your services, and Communicate your support to others. Oklahoma's herbaria need your help to avoid local or regional extinction. A quick perusal of Table 1 indicates that there is a herbarium located conveniently near you. Support your local herbarium; help preserve our botanical heritage!

\section{LITERATURE CITED}

Dalton, R. 2003. Natural history collections in crisis as funding is slashed. Nature 423: 575 .

Funk, V. A. 2003. The importance of herbaria. Plant Science Bulletin 49(3): 94-95.

Gropp, R. W. 2003. Are university natural science collections going extinct? Bioscience 53:550.

Holmgren, P. K., N. H. Holmgren, and L. C. Barnett (eds.). 1990. Index Herbariorum. Bronx, New York: New York Botanical Garden Press.

Raven, P. H. 2003. Biodiversity and the future. American Scientist 91:382

Table. Approximate numbers of specimens housed in the Oklahoma herbarium community

\begin{tabular}{c}
$\begin{array}{c}\text { Index } \\
\text { Herbariorum } \\
\text { acronym }\end{array}$ \\
Institution and location \\
\hline
\end{tabular}

$\begin{array}{ll}\text { Number } & \text { Number of } \\ \text { of total } & \text { Oklahoma }\end{array}$ specimens specimens

CAMU

CSU

DUR

ECSC

NOSU

NWOSU

OCLA

OKL

OKLA

ORU

TULS

$\mathrm{WOH}$
Cameron University, Lawton

University of Central Oklahoma, Edmond

Southeastern Oklahoma State University, Durant

East Central University, Ada

Northeastern State University, Tahlequah

Northwestern Oklahoma State University, Alva

University of Science \& Arts of Oklahoma, Chickasha

University of Oklahoma, Norman

Oklahoma State University, Stillwater

Oral Roberts University, Tulsa

University of Tulsa

Southwestern Oklahoma State University, Woodward

Oklahoma Panhandle State University, Goodwell

TOTALS

\begin{tabular}{cc}
6000 & 5000 \\
10,000 & 9000 \\
20,000 & 12,000 \\
6000 & 6000 \\
6000 & 5500 \\
5000 & 4000 \\
20,000 & 18,000 \\
210,000 & 150,000 \\
140,000 & 112,000 \\
6000 & 5500 \\
10,000 & 8000 \\
11,000 & 10,000 \\
3000 & 2500 \\
\hline $\mathbf{4 5 3 , 0 0 0}$ & $\mathbf{3 4 7 , 5 0 0}$
\end{tabular}

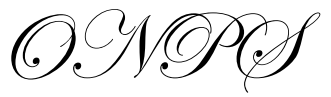

Critic's Choice

Elisens, W.J. 


\title{
Editorial \\ Why Do Species' Names Change?
}

\author{
Patricia A. Folley
}

The reason why scientific names change is because research is constantly correcting errors and scholarship is constantly untangling the related misconceptions. Until the advent of the Internet new names and name changes were approved by the International Botanical Congresses that met at ten-year intervals. Between intervals, proposed new names were published by recognized publications like Rhodora or Sida.

In 1994 John T. Kartesz of the Biota of North American Program published a two-volume second edition of A Synonymized Checklist of the V ascular Flora of the United States, Canada, and Greenland, which became the established reference for names of North American plants on the date of its publication. This work made the Flora of North America project practical by setting a base population against which the specialists could establish the limits of their work.

With this resource there are two transforming innovations that are currently bringing about more rapid name changes in North American flora. First, the advent of the Internet has vastly increased the speed of communication of scientific literature. Results of research are published on the Web within days of their discovery, and search engines make them accessible immediately.

The U.S. Department of Agriculture has long-maintained a database for plant names for use by its agents and agencies. When that database became available on-line, with the inclusion of the Kartesz. checklist, any person with an Internet connection could find out the current status of a plant name within a few minutes. The USDA Plants database http://plants.usda.gov/plants then became the publisher for all additions and corrections to the Kartesz work, and changes are now posted daily. New names and combinations are also still published in print, including a detailed description of the plants involved. The impact on scholarship can be seen as the difference between the old "10 years or so" and the current " 24 hours or so".

The second transformer is the Flora of North America Project (FNA) which was begun in 1982 at the Missouri Botanical Gardens. Since the publication of Vol. 1 in 1993 the Flora project has driven both scholarship and research into the details of floristics in America. The list of contributors includes plant systematists and taxonomists still living today. Conceived as a database project from the beginning, it both feeds and is fed by the Internet.

Standards for the FNA work have always compelled workers to research global archives. Information based on past assumptions required verification, and the verification process yielded unexpected results. Many contributors found themselves revising a lifetime of their own research before it could be accepted into the FNA. Verifying the work of contributors who have passed on is being continued by their successors. The majority of these efforts are being made by scholars and scientists who, while publicly funded for their teaching or research work, are not otherwise supported, and thus are volunteering their time and knowledge.

As users of botanical information, we are often challenged to know what "today's name" for a plant may be. But the outcome of the FNA project, coupled with the unparalleled access to the literature provided by the Internet, has made all of us better scholars with more reliable sources of information on the plants themselves. In time, the FNA project will also become a printed reality, and the rate of change will slow. However, it will never cease as long as the real plants out in the real world continue to evolve. 


\section{Editorial Policies and Practices}

Oklahoma Native Plant Record is published annually by Oklahoma Native Plant Society. Submission for publication in the journal is open to all. Manuscripts will be accepted on topics related to Oklahoma's regional botany, including historical research reports, current research articles, site record species lists, and descriptions of new or important species sightings in Oklahoma. Oklahoma's environmental gradients of human impact, climate, and elevation make us a prime target for research on habitat edges, species ranges, and edge species, but articles of other themes may be included as well. Important works overlooked by journals of broader geographic regions will also be considered for publication here.

Papers must not have been published previously or accepted for submission elsewhere and should represent research conducted in accordance with accepted procedures and scientific ethics. Submission of the article implies the granting of copyright permission to Oklahoma Native Plant Society.

Manuscripts will be reviewed for content and appropriateness by at least two reviewers. The title page should state the affiliation and complete addresses of all authors and telephone numbers for the corresponding author. Research and technical papers should include a one-paragraph abstract of not more than 250 words. It should concisely state the goals, principal results, and major conclusions of the paper. All references, figures, and tables should be cited in the text. Common names should be referenced to a scientific name. Abbreviations of authorities for scientific names should follow Authors of Plant Names (Brummitt and Powell, 1992). Titles of periodicals should be abbreviated following Botanico-Peridoicum-Huntianum and its supplement - except in historic publications when original format will be used.

Authors with access to IBM-compatible microcomputers are encouraged to send a copy of the manuscript on diskette in $\mathrm{rtf}$ (rich text format). If the manuscript is typed, manuscripts should be double-spaced on $8^{1 / 2} X 11$ inch paper with minimum one-inch margins and should be submitted in duplicate. Diskette or hardcopy manuscripts should be sent to the managing editor at the ONPS address on the back cover by June 1.

Oklahoma Native Plant Society

c/o Tulsa Garden Center

2435 South Peoria

Tulsa, Oklahoma 74114 
Oklahoma Native Plant Society

c/o Tulsa Garden Center

2435 South Peoria

Tulsa, Oklahoma 74114
Non-Profit

Organization

U.S. Postage Paid

Tulsa, OK

Permit No. 357

In this issue of Oklahoma Native Plant Record Volume 4, Number 1, December 2004:

4 Ecological factors affecting the distribution of woody vegetation near the Arkansas

River, Tulsa County, Anne Wanamaker Long

24 Cotinus obovatus Raf. (Smoke-tree) in Oklahoma, Bruce Hoagland

26 Giant cane and southeastern Indian baskets, Julia A. Jordan

30 Vascular flora of the Chouteau Wildlife Management Area, Wagoner County, Oklahoma, Bruce W. Hoagland and Forrest L. Johnson (dec.)

40 Cypripedium, Bruce W. Hoagland and Amy K. Buthod

48 Common lawn and garden mushrooms of central Oklahoma, Clark L. Ovrebo

55 Critic's Choice Essay: Support Your Local Herbarium, Wayne J. Elisens

57 Why do species names change? Patricia A. Folley 